\title{
Daphniyunnines A-E, Five New Alkaloids from Daphniphyllum yunnanense
}

Hua Zhang, Sheng-Ping Yang, Cheng-Qi Fan, Jian Ding, and Jian-Min Yue*

State Key Laboratory of Drug Research, Institute of Materia Medica, Shanghai Institutes for Biological Sciences, Chinese Academy of Sciences, 555 Zu Chong Zhi Road, Zhangjiang Hi-Tech Park, Shanghai, 201203, P. R. China

\section{Supporting Information}

1. Page 4-56, NMR data of the daphniyunnine A - E (1-5)

Figure S1. ${ }^{1} \mathrm{H}$ NMR spectrum of daphniyunnine $\mathrm{A}(\mathbf{1})$ in $\mathrm{CD}_{3} \mathrm{OD}$.

Figure S2. ${ }^{13} \mathrm{C}$ NMR spectrum of daphniyunnine $\mathrm{A}(\mathbf{1})$ in $\mathrm{CD}_{3} \mathrm{OD}$.

Figure S3. Expanded ${ }^{13} \mathrm{C}$ NMR spectrum of daphniyunnine $\mathrm{A}(\mathbf{1})$ in $\mathrm{CD}_{3} \mathrm{OD}\left(\delta_{\mathrm{C}} 17-70\right)$.

Figure S4. ${ }^{13} \mathrm{C}$ NMR (DEPT) spectrum of daphniyunnine A (1) in $\mathrm{CDCl}_{3}$.

Figure S5. EIMS spectrum of daphniyunnine A (1).

Figure S6. IR spectrum of daphniyunnine A (1).

Figure S7. ${ }^{1} \mathrm{H}-{ }^{1} \mathrm{H}$ COSY spectrum of daphniyunnine $\mathrm{A}(\mathbf{1})$ in $\mathrm{CD}_{3} \mathrm{OD}$.

Figure S8. HMQC spectrum of daphniyunnine A (1) in $\mathrm{CD}_{3} \mathrm{OD}$.

Figure S9. HMBC spectrum of daphniyunnine A (1) in $\mathrm{CD}_{3} \mathrm{OD}$.

Figure S10. NOESY spectrum of daphniyunnine $\mathrm{A}(\mathbf{1})$ in $\mathrm{CD}_{3} \mathrm{OD}$.

Figure S11. ${ }^{1} \mathrm{H}$ NMR spectrum of daphniyunnine B (2) in $\mathrm{CDCl}_{3}+\mathrm{CD}_{3} \mathrm{OD}$.

Figure S12. ${ }^{13} \mathrm{C}$ NMR spectrum of daphniyunnine B (2) in $\mathrm{CDCl}_{3}+\mathrm{CD}_{3} \mathrm{OD}$.

Figure S13. Expanded ${ }^{13} \mathrm{C}$ NMR spectrum of daphniyunnine $\mathrm{B}(2)$ in $\mathrm{CDCl}_{3}+\mathrm{CD}_{3} \mathrm{OD}\left(\delta_{\mathrm{C}} 17-73\right)$.

Figure S14. DEPT spectrum of daphniyunnine B (2) in $\mathrm{CDCl}_{3}+\mathrm{CD}_{3} \mathrm{OD}$.

Figure S15. EIMS spectrum of daphniyunnine B (2).

Figure S16. IR spectrum of daphniyunnine B (2).

${ }^{*}$ Corresponding author. Tel.: +86-21-50806718, Fax: +86-21-50806718. 
Figure S17. ${ }^{1} \mathrm{H}-{ }^{1} \mathrm{H}$ COSY spectrum of daphniyunnine B (2) in $\mathrm{CDCl}_{3}+\mathrm{CD}_{3} \mathrm{OD}$.

Figure S18. HMQC spectrum of daphniyunnine B (2) in $\mathrm{CDCl}_{3}+\mathrm{CD}_{3} \mathrm{OD}$.

Figure S19. HMBC spectrum of daphniyunnine B (2) in $\mathrm{CDCl}_{3}+\mathrm{CD}_{3} \mathrm{OD}$.

Figure S20. NOESY spectrum of daphniyunnine B (2) in $\mathrm{CDCl}_{3}+\mathrm{CD}_{3} \mathrm{OD}$.

Figure S21. ${ }^{1} \mathrm{H}$ NMR spectrum of daphniyunnine C (3) in $\mathrm{CDCl}_{3}$.

Figure S22. ${ }^{13} \mathrm{C}$ NMR spectrum of daphniyunnine $\mathrm{C}(3)$ in $\mathrm{CDCl}_{3}$.

Figure S23. Expanded ${ }^{13} \mathrm{C}$ NMR spectrum of daphniyunnine $\mathrm{C}(3)$ in $\mathrm{CDCl}_{3}\left(\delta_{\mathrm{C}} 42-68\right)$.

Figure S24. DEPT spectrum of daphniyunnine C (3) in $\mathrm{CDCl}_{3}$.

Figure S25. Expanded DEPT spectrum of daphniyunnine C (3) in $\mathrm{CDCl}_{3}\left(\delta_{\mathrm{C}} 42-68\right)$.

Figure S26. EIMS spectrum of daphniyunnine C (3).

Figure S27. IR spectrum of daphniyunnine C (3).

Figure S28. ${ }^{1} \mathrm{H}-{ }^{1} \mathrm{H}$ COSY spectrum of daphniyunnine C (3) in $\mathrm{CDCl}_{3}$.

Figure S29. Expanded ${ }^{1} \mathrm{H}-{ }^{1} \mathrm{H}$ COSY spectrum of daphniyunnine C (3) in $\mathrm{CDCl}_{3}\left(\delta_{\mathrm{H}} 0.8-4.3\right)$.

Figure S30. HMQC spectrum of daphniyunnine C (3) in $\mathrm{CDCl}_{3}$.

Figure S31. HMBC spectrum of daphniyunnine C (3) in $\mathrm{CDCl}_{3}$.

Figure S32. NOESY spectrum of daphniyunnine C (3) in $\mathrm{CDCl}_{3}$.

Figure S33. Expanded NOESY spectrum of daphniyunnine C (3) in $\mathrm{CDCl}_{3}\left(\delta_{\mathrm{H}} 0.9-4.3\right)$.

Figure S34. ${ }^{1} \mathrm{H}$ NMR spectrum of daphniyunnine D (4) in $\mathrm{CDCl}_{3}$.

Figure S35. ${ }^{13} \mathrm{C}$ NMR spectrum of daphniyunnine $\mathrm{D}(4)$ in $\mathrm{CDCl}_{3}$.

Figure S36. Expanded ${ }^{13} \mathrm{C}$ NMR spectrum of daphniyunnine $\mathrm{D}(4)$ in $\mathrm{CDCl}_{3}\left(\delta_{\mathrm{C}} 13-66\right)$.

Figure S37. DEPT spectrum of daphniyunnine D (4) in $\mathrm{CDCl}_{3}$.

Figure S38. EIMS spectrum of daphniyunnine D (4).

Figure S39. IR spectrum of daphniyunnine D (4).

Figure S40. ${ }^{1} \mathrm{H}-{ }^{1} \mathrm{H}$ COSY spectrum of daphniyunnine D (4) in $\mathrm{CDCl}_{3}$. 
Figure S41. HMQC spectrum of daphniyunnine D (4) in $\mathrm{CDCl}_{3}$.

Figure S42. HMBC spectrum of daphniyunnine D (4) in $\mathrm{CDCl}_{3}$.

Figure S43. NOESY spectrum of daphniyunnine D (4) in $\mathrm{CDCl}_{3}$.

Figure S44. ${ }^{1} \mathrm{H}$ NMR spectrum of daphniyunnine E (5) in $\mathrm{CDCl}_{3}$.

Figure S45. ${ }^{13} \mathrm{C}$ NMR spectrum of daphniyunnine $\mathrm{E}(5)$ in $\mathrm{CDCl}_{3}$.

Figure S46. Expanded ${ }^{13} \mathrm{C}$ NMR spectrum of daphniyunnine $\mathrm{E}(5)$ in $\mathrm{CDCl}_{3}\left(\delta_{\mathrm{C}} 47-67\right)$.

Figure S47. DEPT spectrum of daphniyunnine E (5) in $\mathrm{CDCl}_{3}$.

Figure S48. EIMS spectrum of daphniyunnine E (5).

Figure S49. IR spectrum of daphniyunnine E (5).

Figure S50. ${ }^{1} \mathrm{H}-{ }^{1} \mathrm{H}$ COSY spectrum of daphniyunnine E (5) in $\mathrm{CDCl}_{3}$.

Figure S51. HMQC spectrum of daphniyunnine E (5) in $\mathrm{CDCl}_{3}$.

Figure S52. HMBC spectrum of daphniyunnine E (5) in $\mathrm{CDCl}_{3}$.

Figure S53. NOESY spectrum of daphniyunnine $\mathrm{E}(5)$ in $\mathrm{CDCl}_{3}$.

2. Page 57-67, X-ray data tables for daphniyunnine A (1) 
Figure S1. ${ }^{1} \mathrm{H}$ NMR spectrum of daphniyunnine $\mathrm{A}(\mathbf{1})$ in $\mathrm{CD}_{3} \mathrm{OD}$

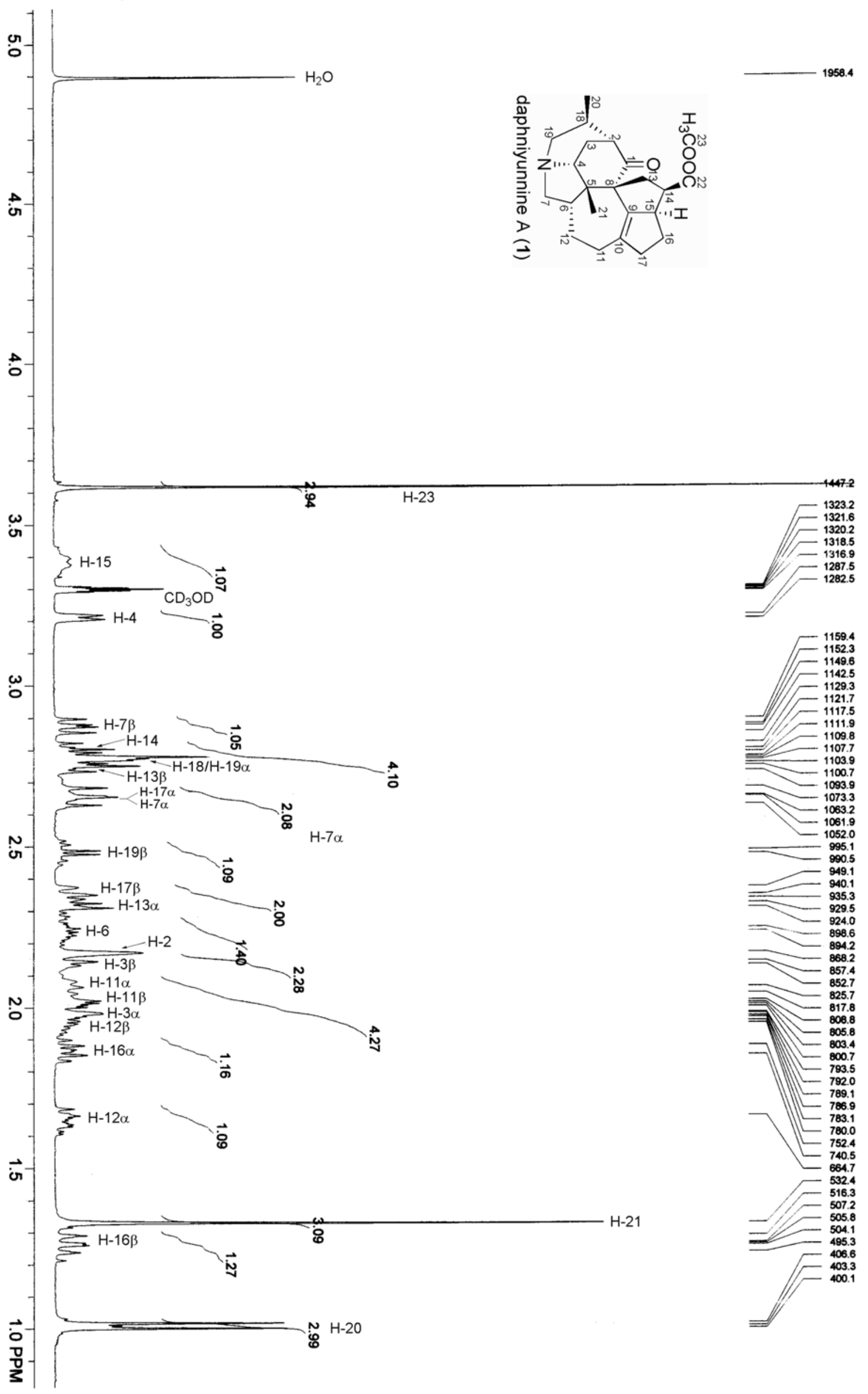


Figure S2. ${ }^{13} \mathrm{C}$ NMR spectrum of daphniyunnine $\mathrm{A}(\mathbf{1})$ in $\mathrm{CD}_{3} \mathrm{OD}$

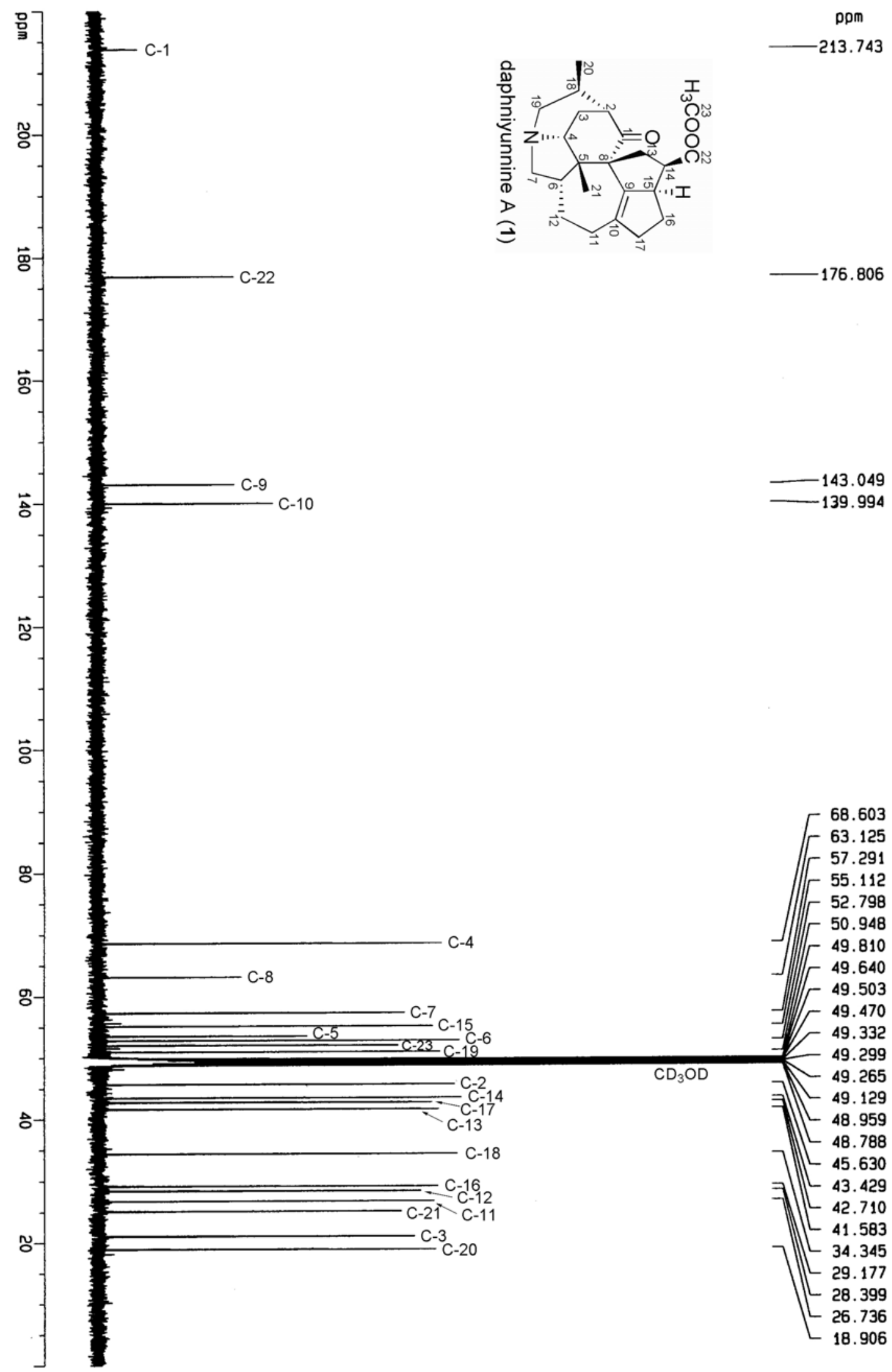


Figure S3. Expanded ${ }^{13} \mathrm{C}$ NMR spectrum of daphniyunnine $\mathrm{A}(\mathbf{1})$ in $\mathrm{CD}_{3} \mathrm{OD}\left(\delta_{\mathrm{C}} 17-70\right)$

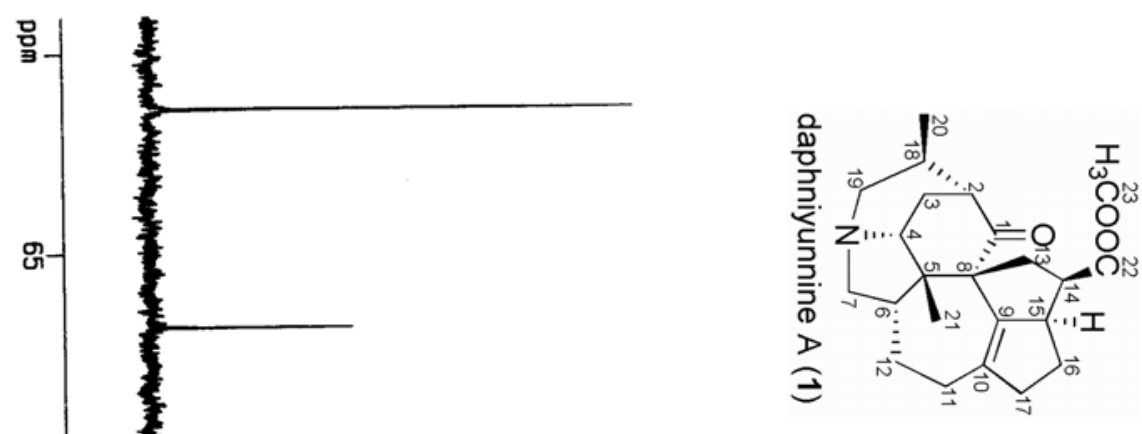

ppm

$-68.6027$

un

ปั

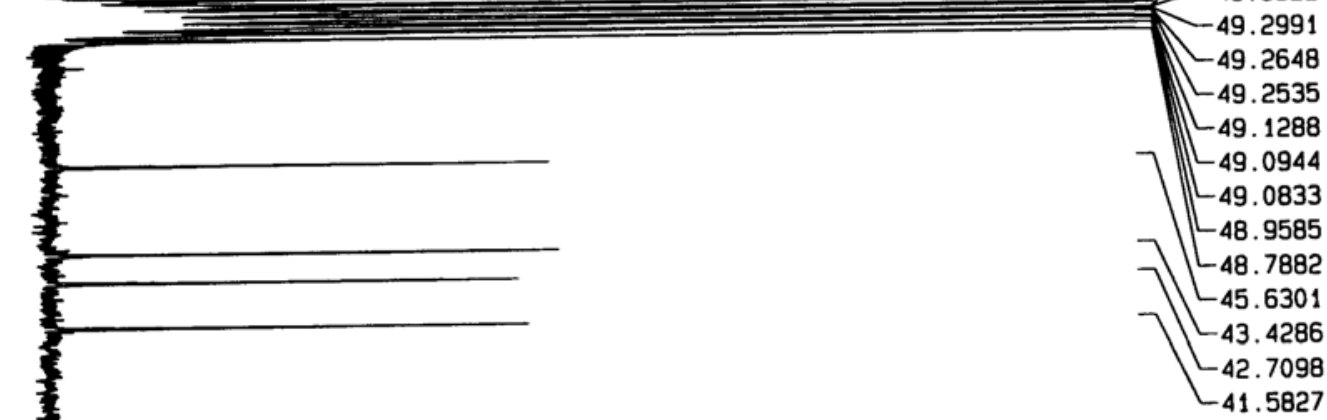

음

41.5827

$-34.3453$

w

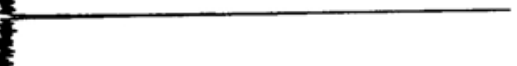

$\omega$
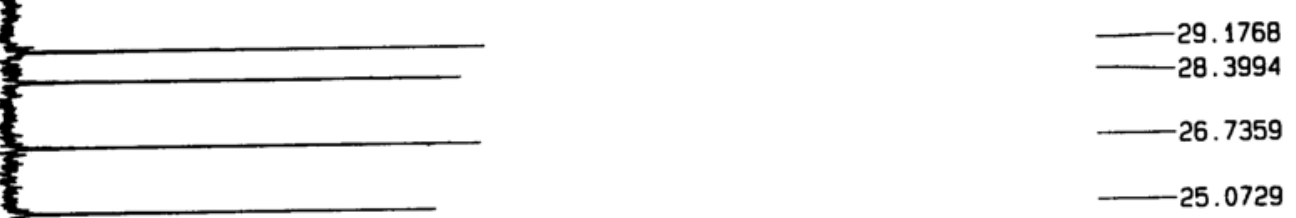

ज-

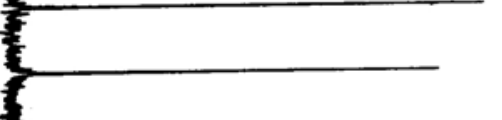

$-21.0224$

ก-

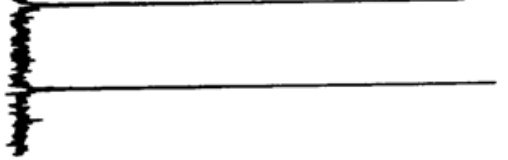

18.9065 
Figure S4. ${ }^{13} \mathrm{C}$ NMR (DEPT) spectrum of daphniyunnine A (1) in $\mathrm{CDCl}_{3}$
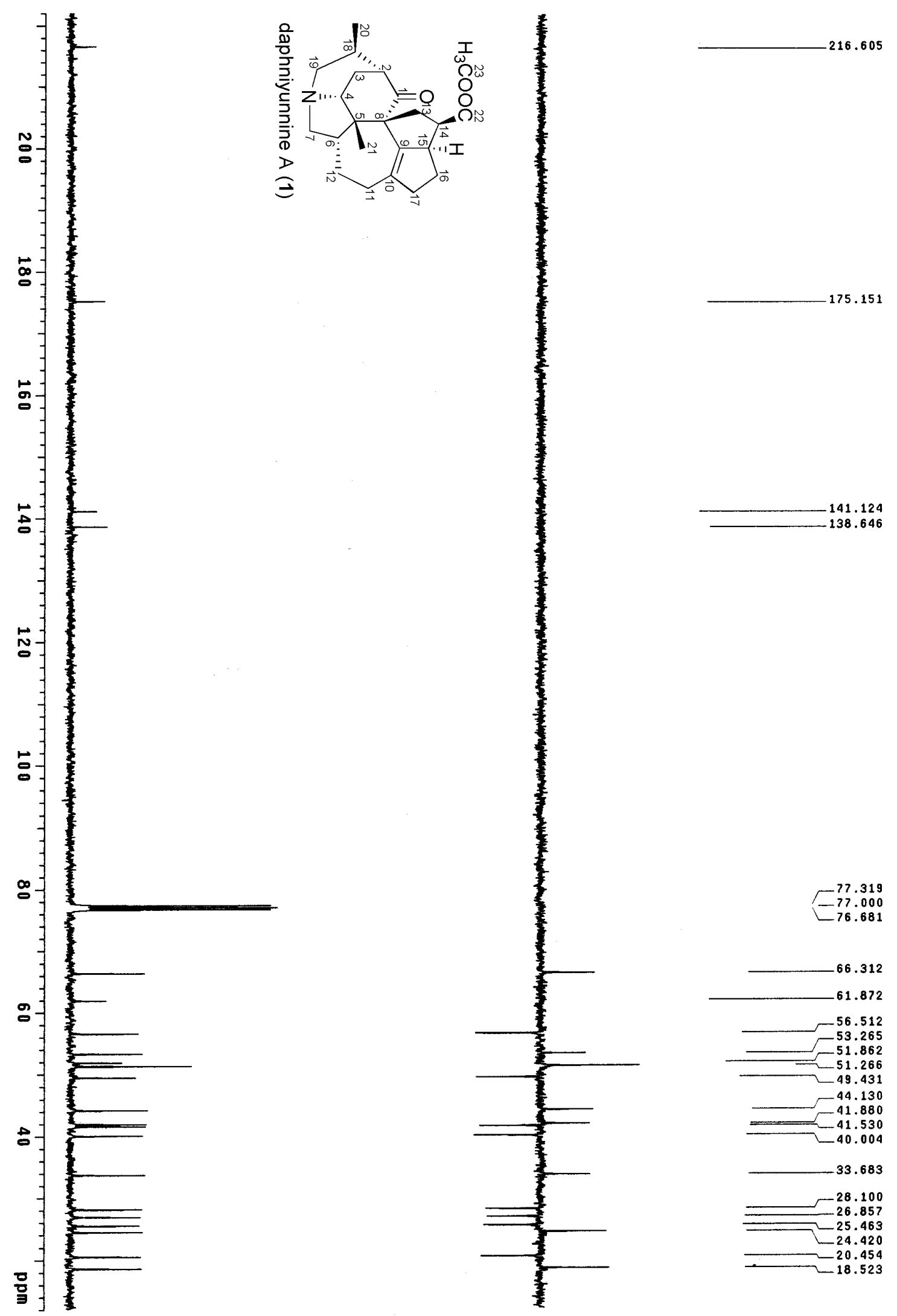

41.124

138.646

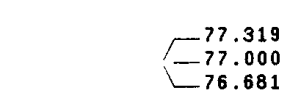

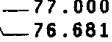

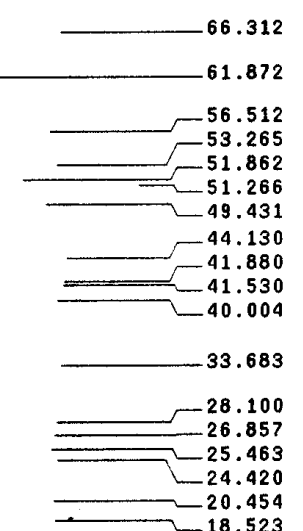


Figure S5. EIMS spectrum of daphniyunnine A (1)

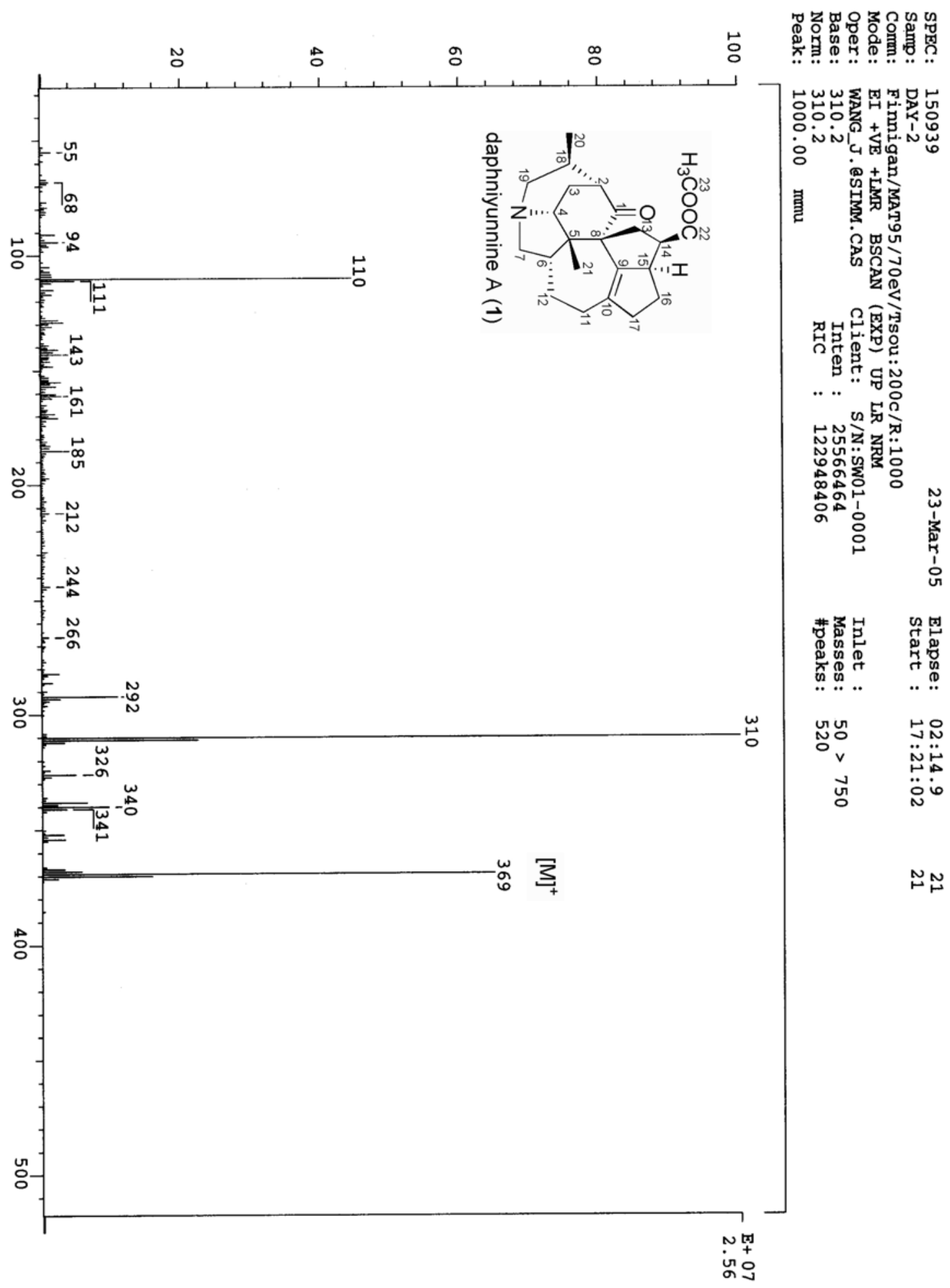


Figure S6. IR spectrum of daphniyunnine A (1)

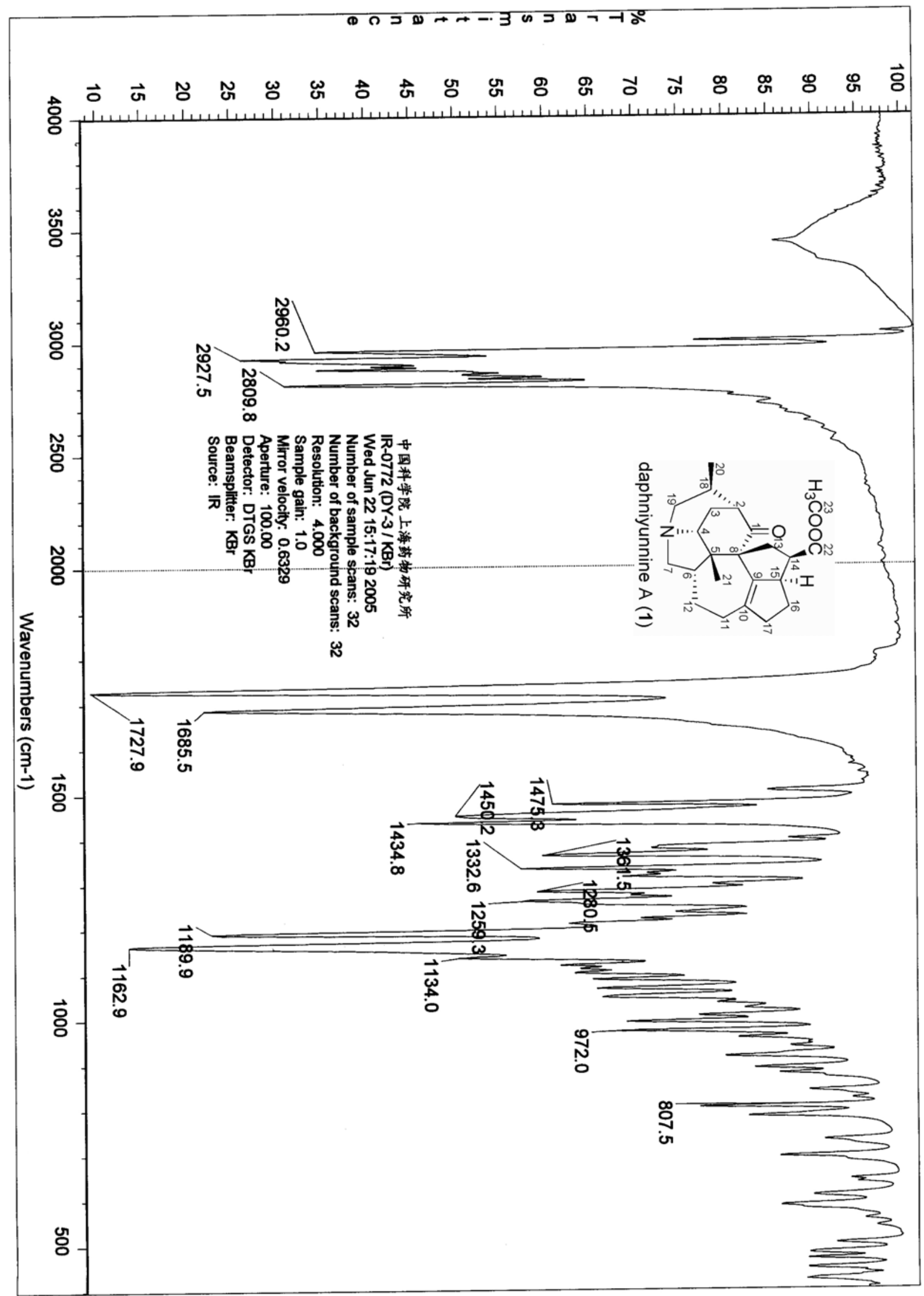


Figure S7. ${ }^{1} \mathrm{H}-{ }^{1} \mathrm{H}$ COSY spectrum of daphniyunnine $\mathrm{A}(\mathbf{1})$ in $\mathrm{CD}_{3} \mathrm{OD}$

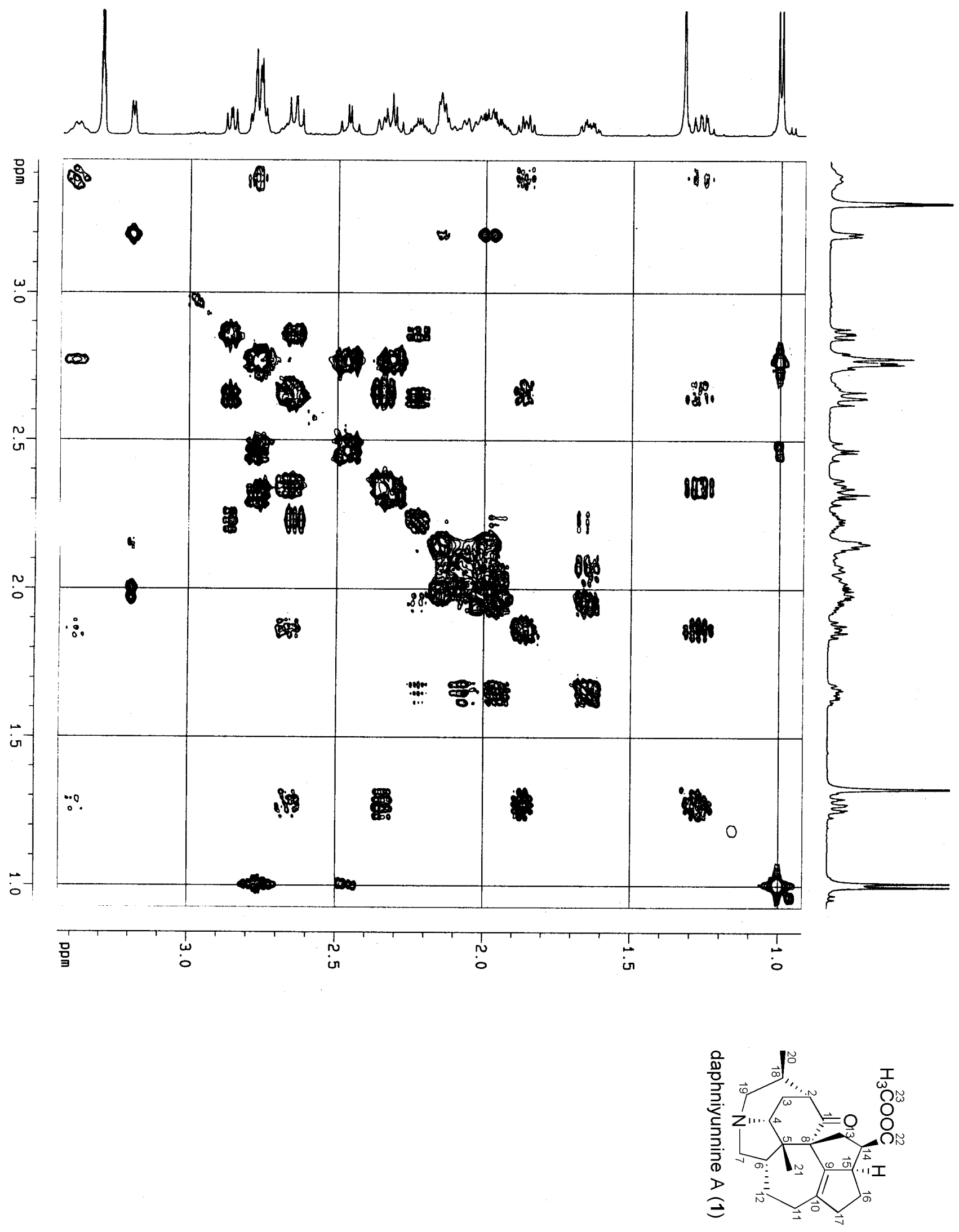


Figure S8. HMQC spectrum of daphniyunnine A (1) in $\mathrm{CD}_{3} \mathrm{OD}$
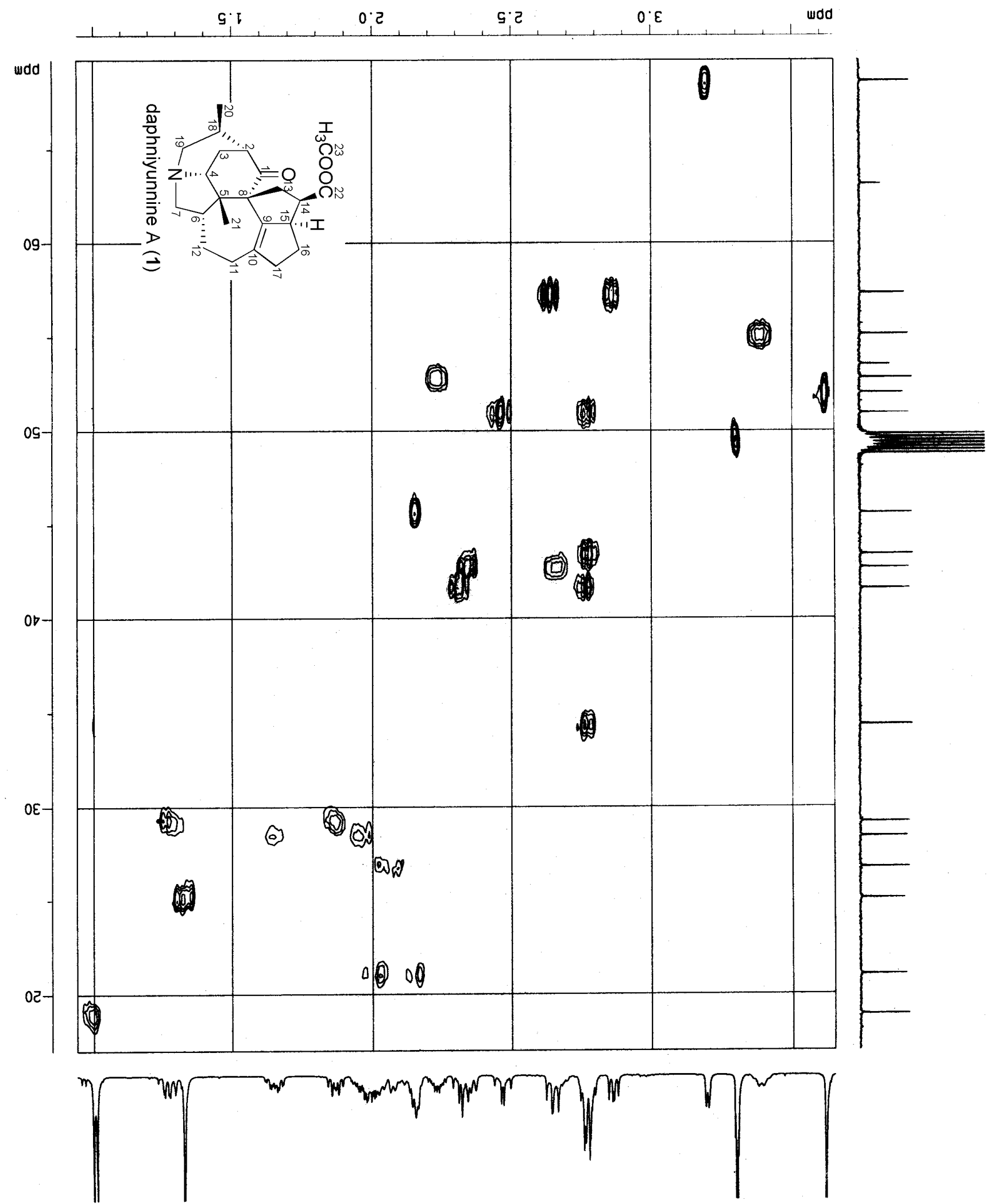
Figure S9. HMBC spectrum of daphniyunnine $\mathrm{A}(\mathbf{1})$ in $\mathrm{CD}_{3} \mathrm{OD}$

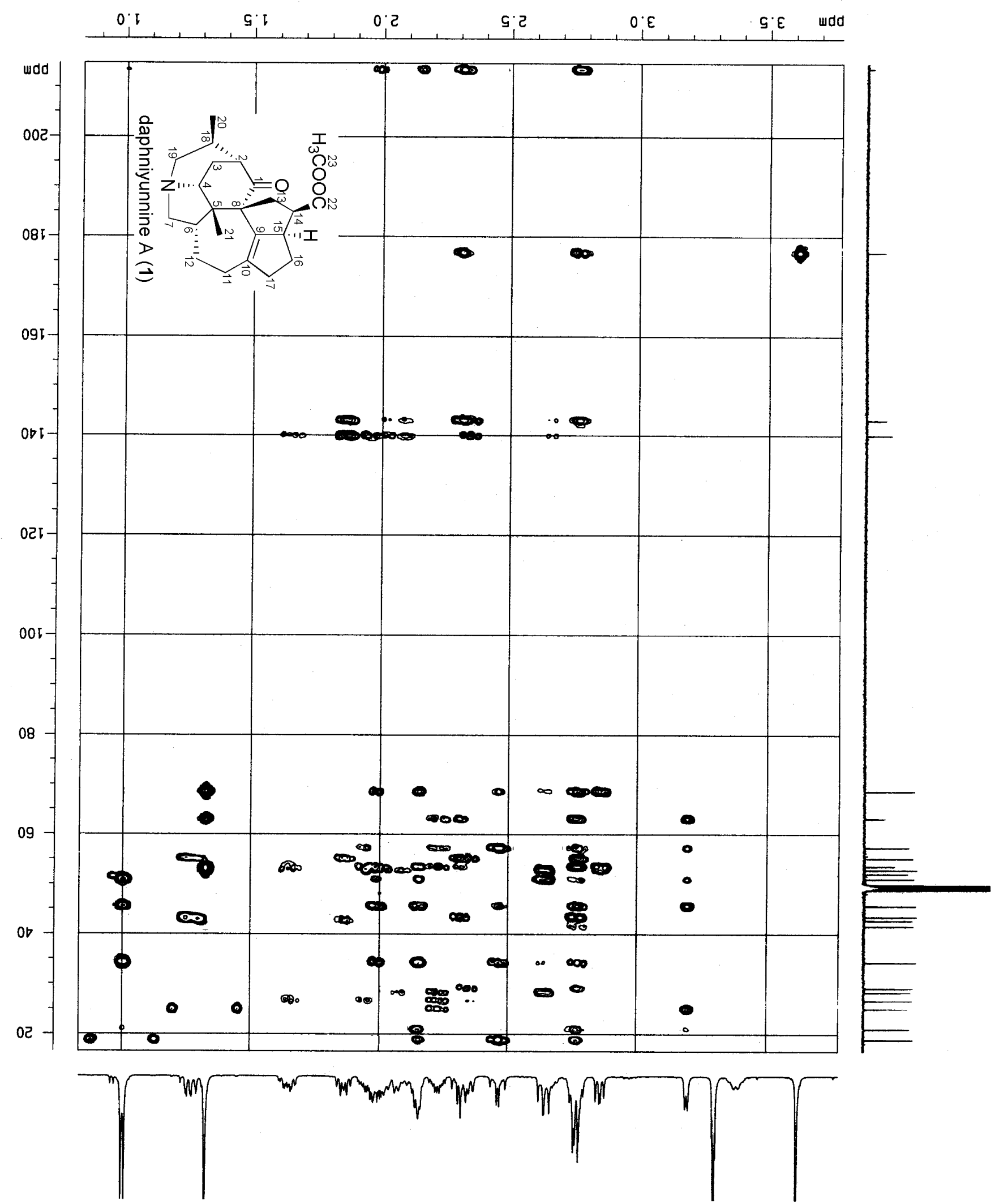


Figure S10. NOESY spectrum of daphniyunnine $\mathrm{A}(\mathbf{1})$ in $\mathrm{CD}_{3} \mathrm{OD}$

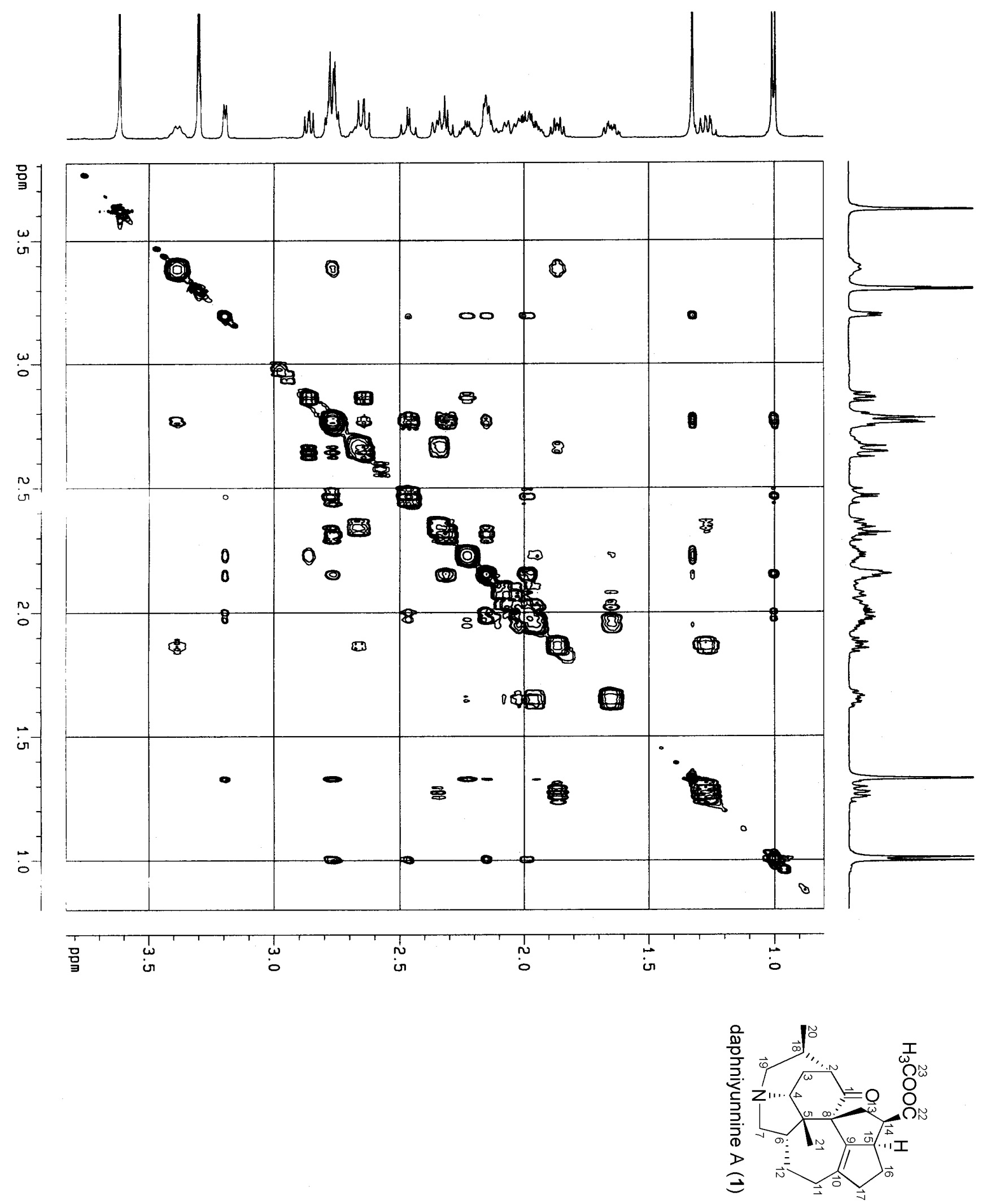


Figure S11. ${ }^{1} \mathrm{H}$ NMR spectrum of daphniyunnine B (2) in $\mathrm{CDCl}_{3}+\mathrm{CD}_{3} \mathrm{OD}$
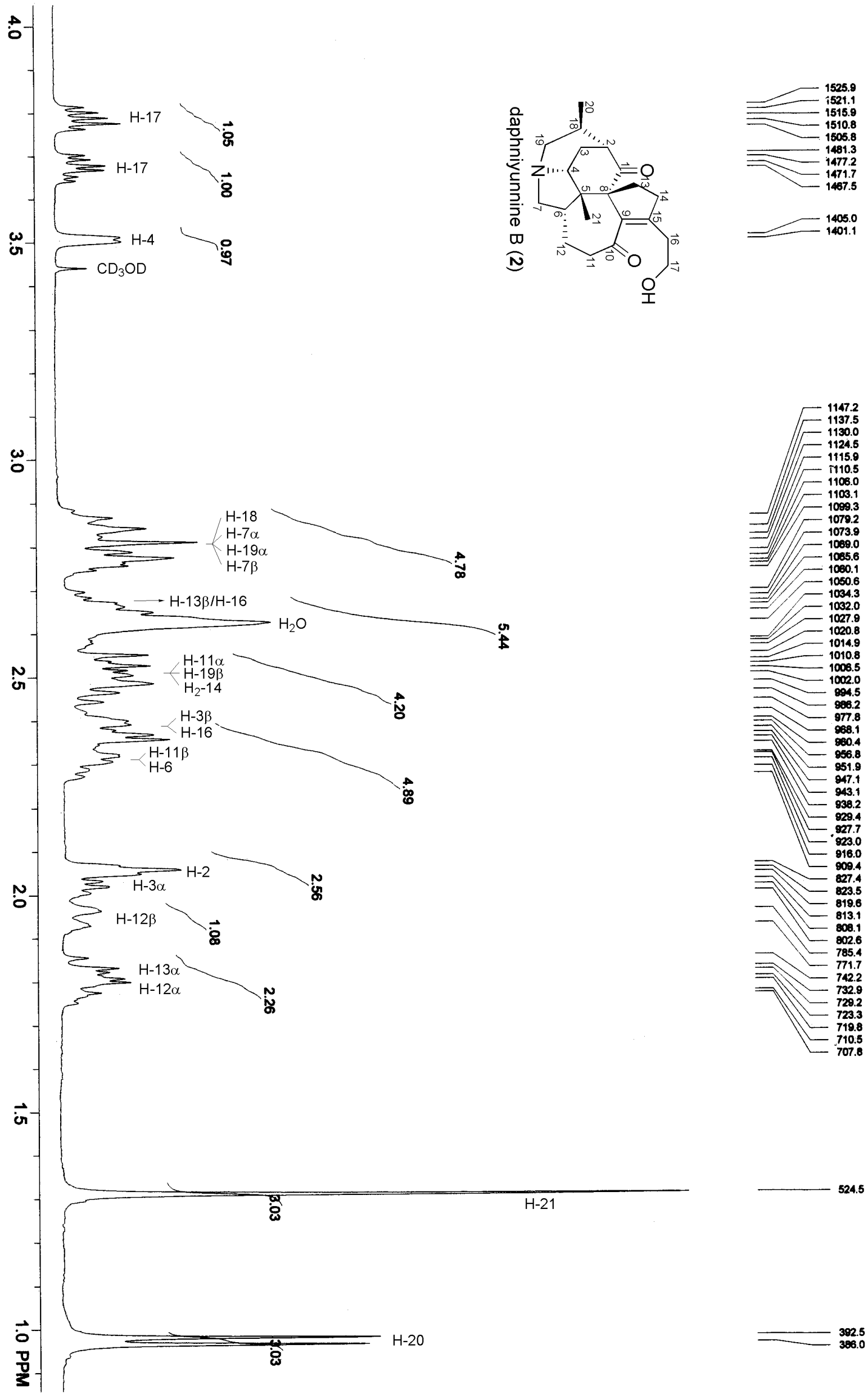

524.5 
Figure S12. ${ }^{13} \mathrm{C}$ NMR spectrum of daphniyunnine B (2) in $\mathrm{CDCl}_{3}+\mathrm{CD}_{3} \mathrm{OD}$

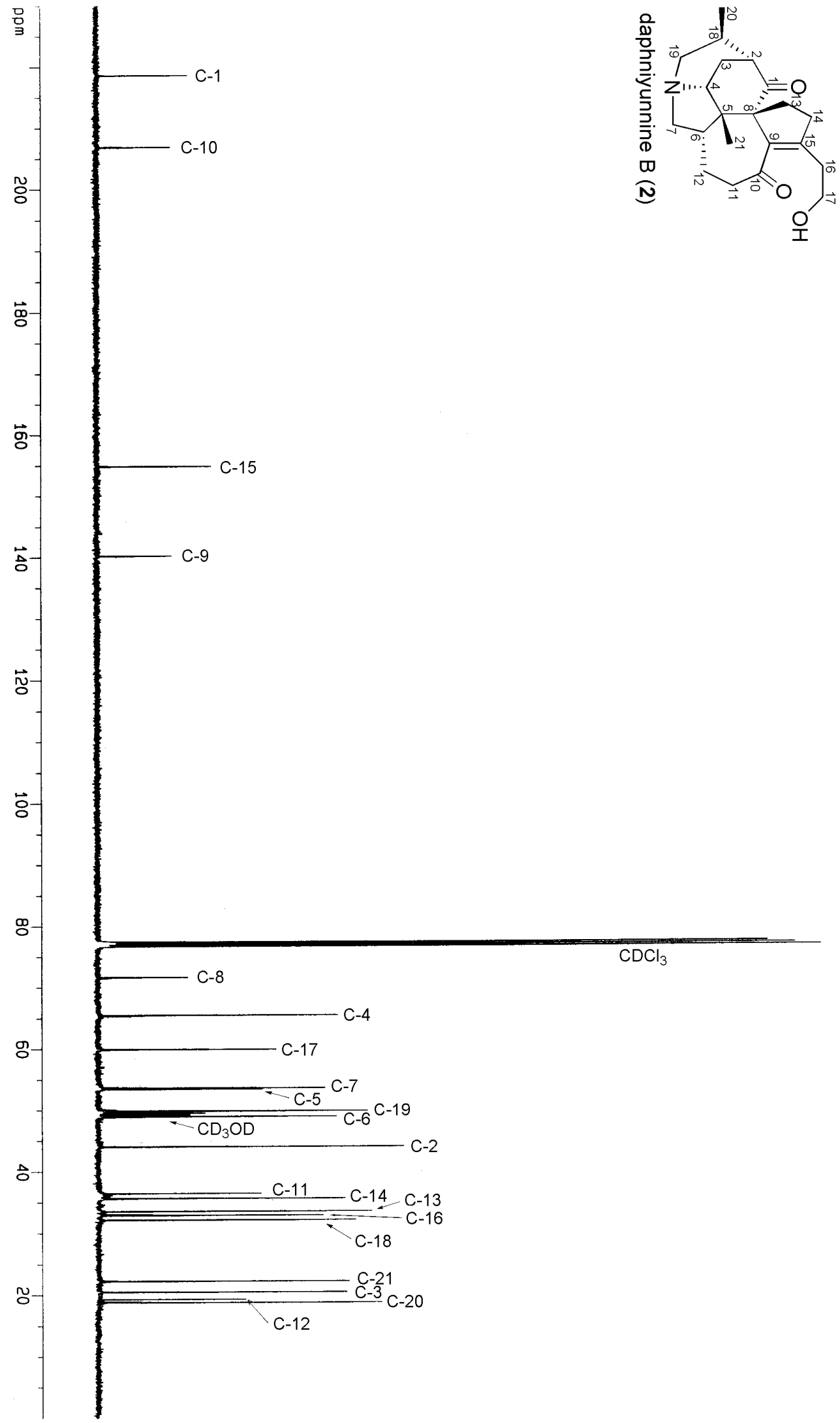

ppm

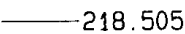

206.789

$-154.816$

$-140.154$

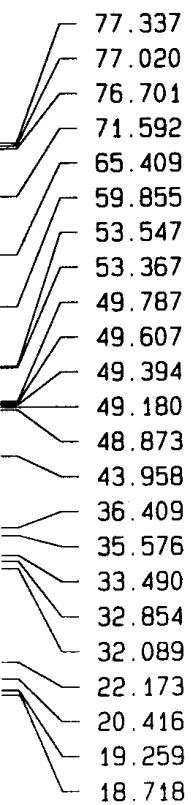


Figure S13. Expanded ${ }^{13} \mathrm{C}$ NMR spectrum of daphniyunnine $\mathrm{B}(2)$ in $\mathrm{CDCl}_{3}+\mathrm{CD}_{3} \mathrm{OD}\left(\delta_{\mathrm{C}} 17-73\right)$
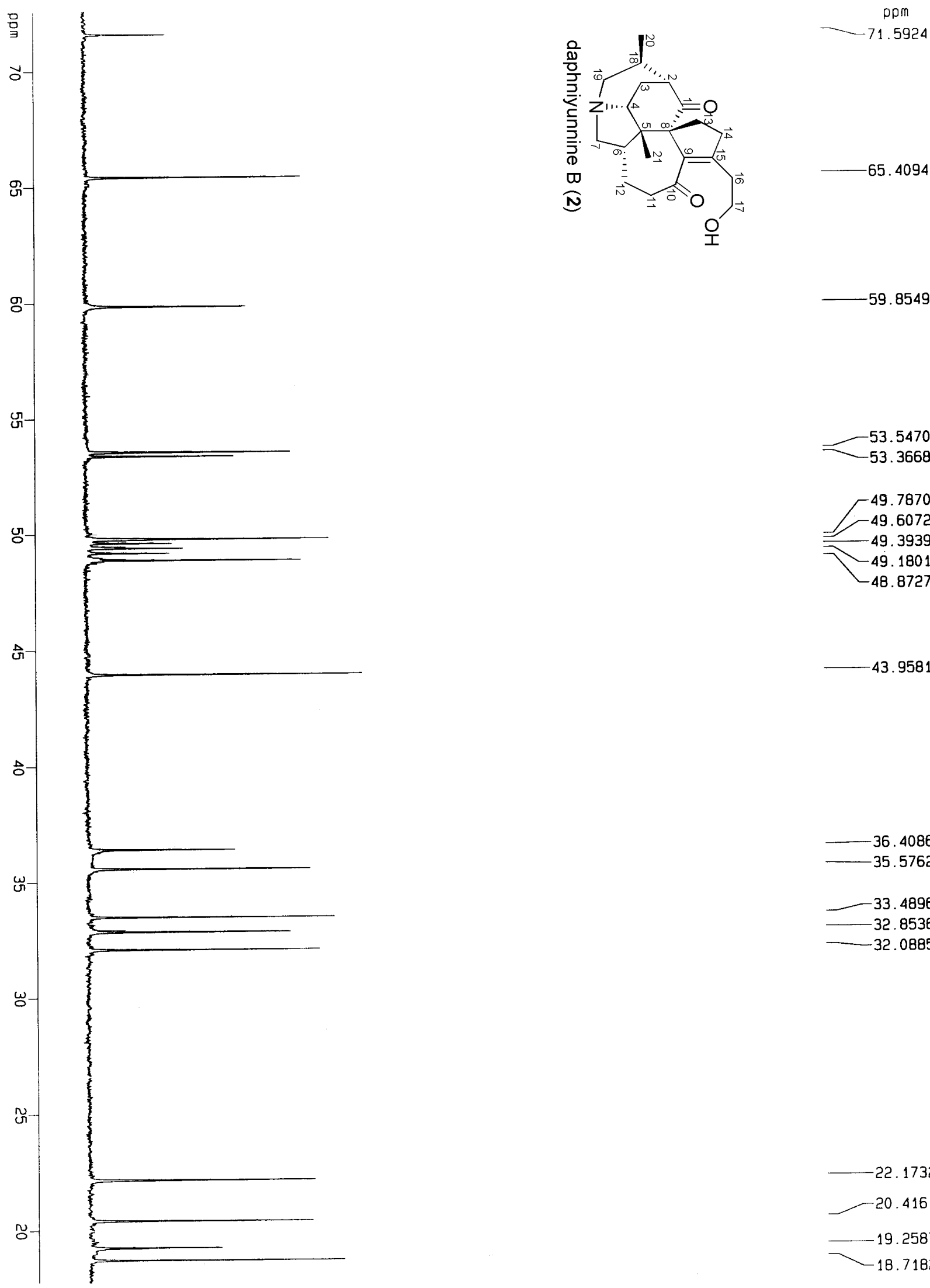

$-59.8549$

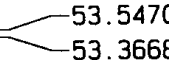

49.7870

49.6072

49. 3939

49.1801

$-48.8727$

43. 9581

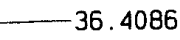

35.5762

33.4896

32.8536

- 32.0885

- - -22.1732

$-20.4161$

$-19.2587$

$-18.7182$ 
Figure S14. DEPT spectrum of daphniyunnine B (2) in $\mathrm{CDCl}_{3}+\mathrm{CD}_{3} \mathrm{OD}$

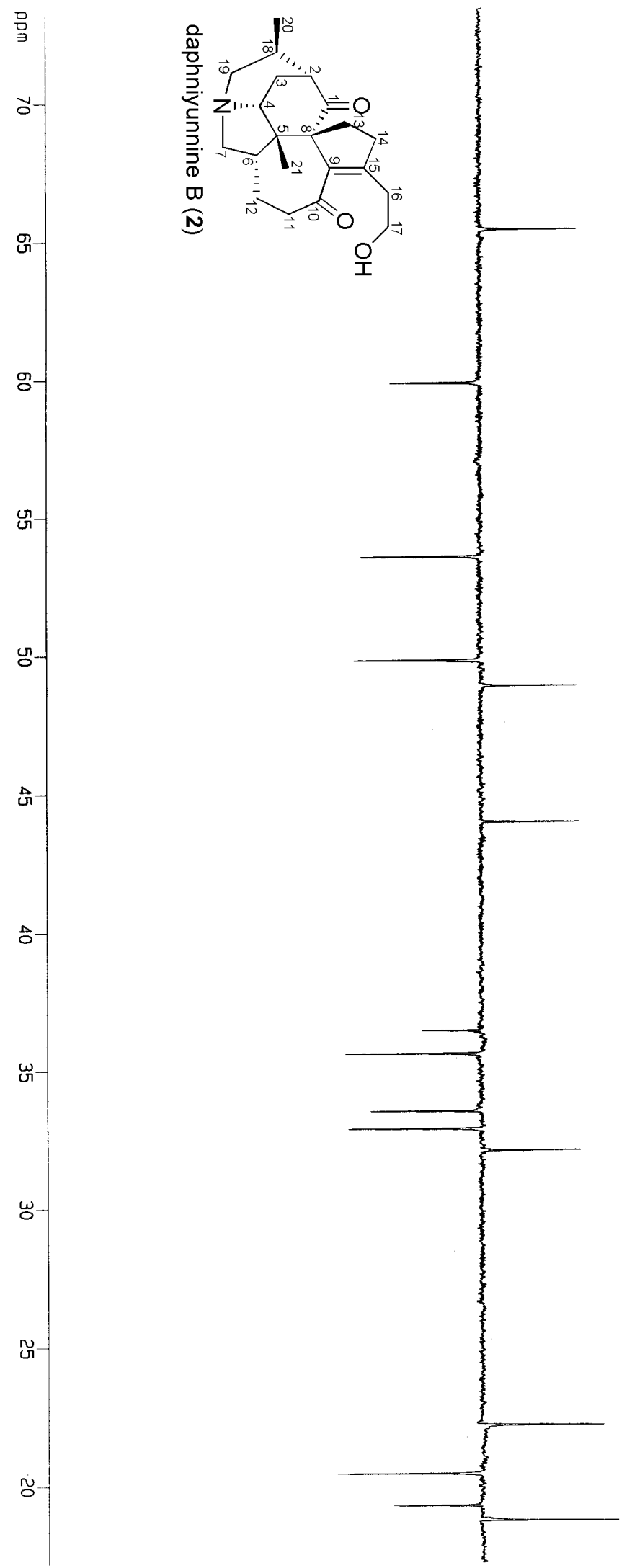

ppm

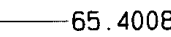

$-59.8511$

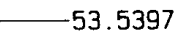

$-49.7807$

48.8680

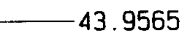

— 36.4070

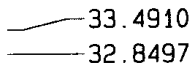

$-2-3$

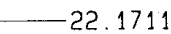

20.4077

19.2545

$\longrightarrow$
$\square$
$\square$ 
Figure S15. EIMS spectrum of daphniyunnine B (2)

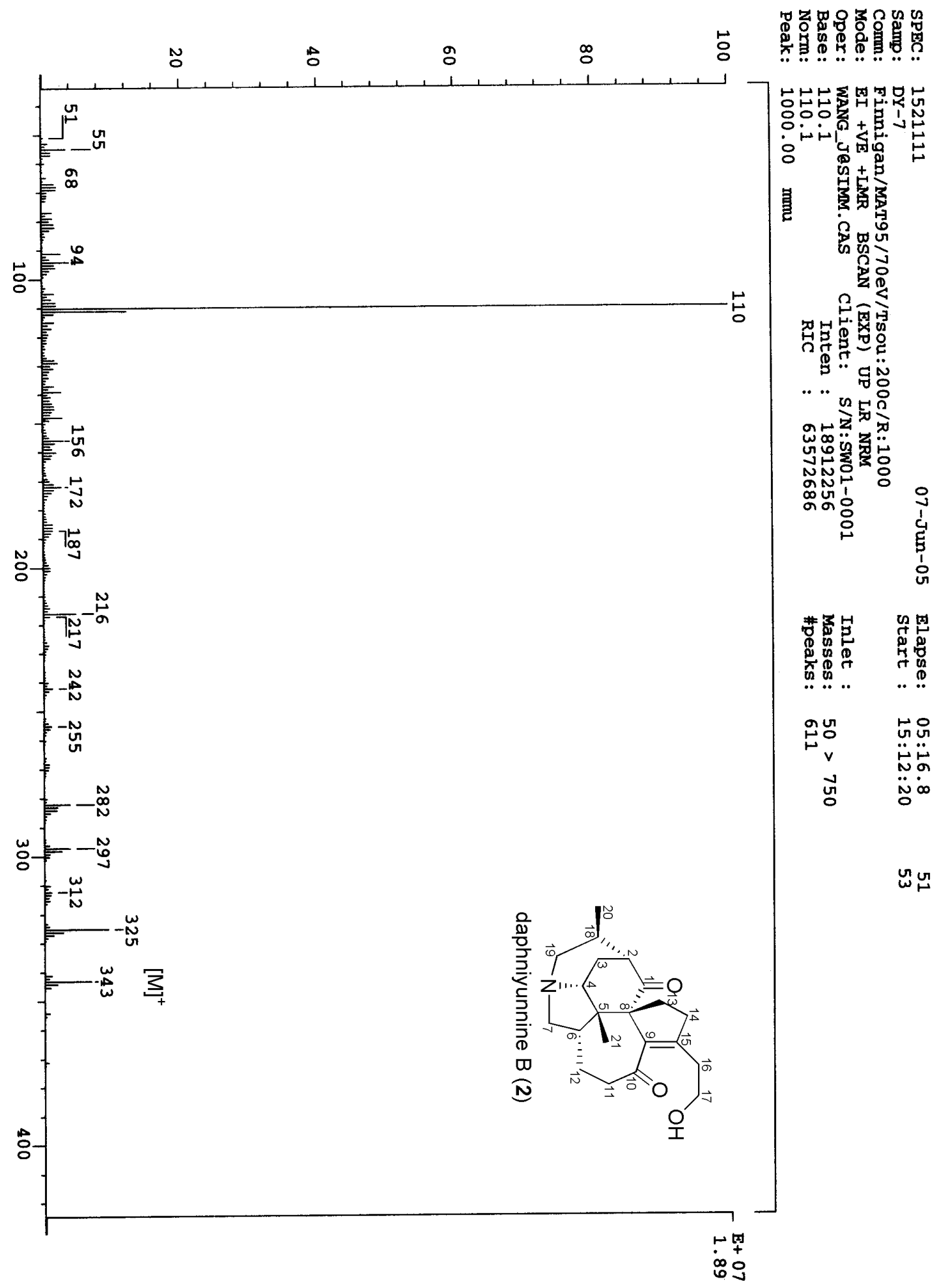


Figure S16. IR spectrum of daphniyunnine B (2)

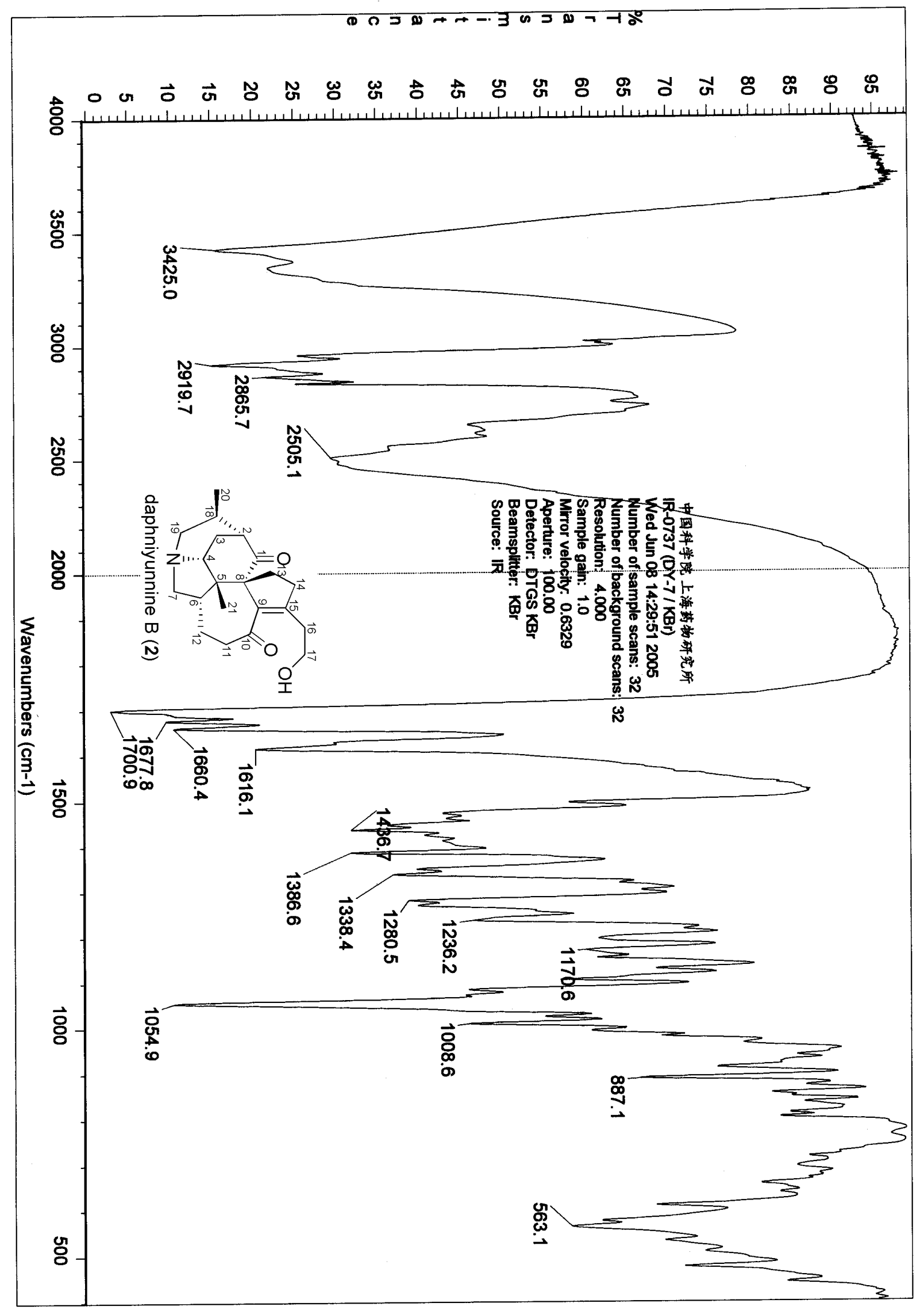


Figure S17. ${ }^{1} \mathrm{H}-{ }^{1} \mathrm{H}$ COSY spectrum of daphniyunnine B (2) in $\mathrm{CDCl}_{3}+\mathrm{CD}_{3} \mathrm{OD}$

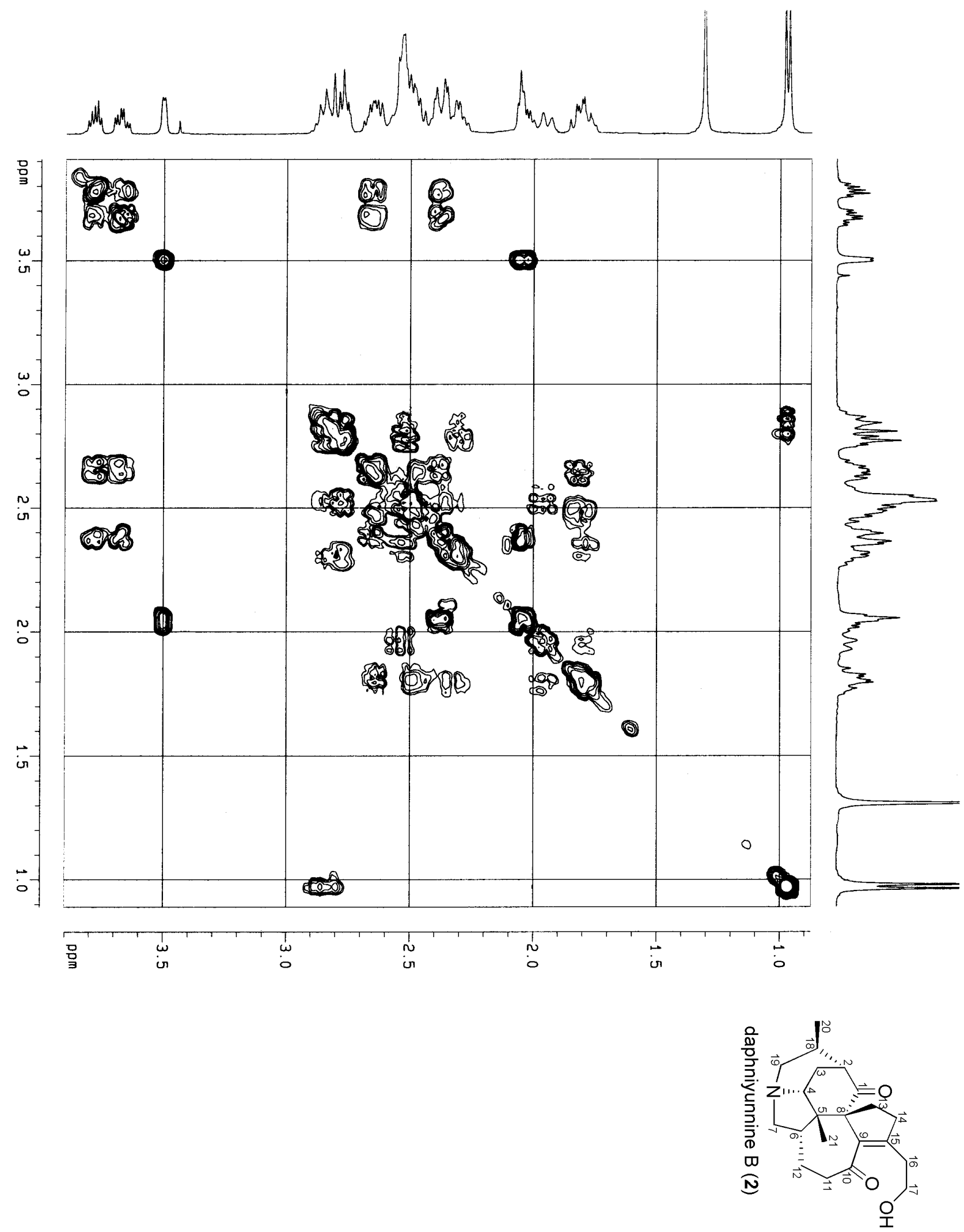


Figure S18. HMQC spectrum of daphniyunnine B (2) in $\mathrm{CDCl}_{3}+\mathrm{CD}_{3} \mathrm{OD}$

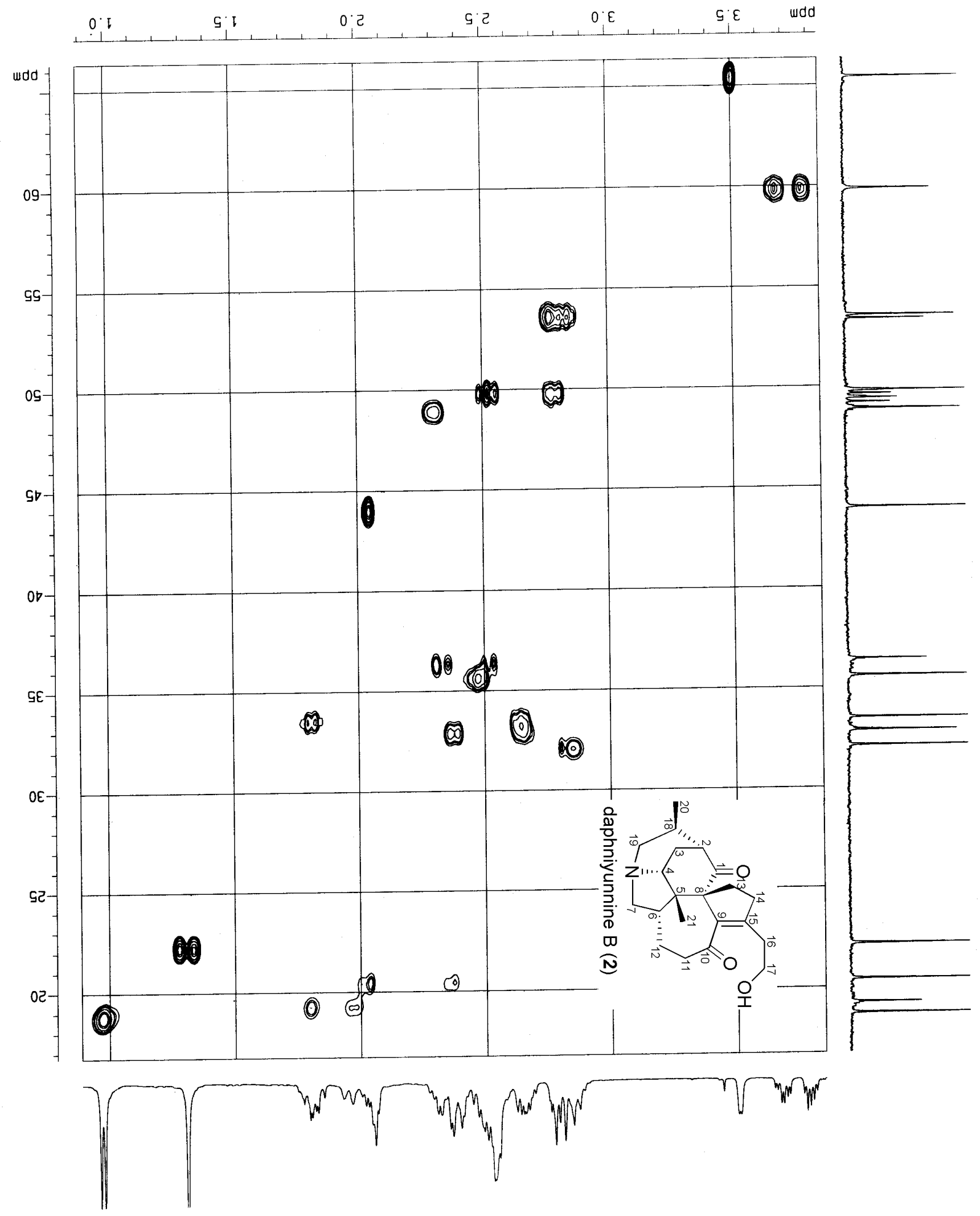


Figure S19. HMBC spectrum of daphniyunnine B (2) in $\mathrm{CDCl}_{3}+\mathrm{CD}_{3} \mathrm{OD}$

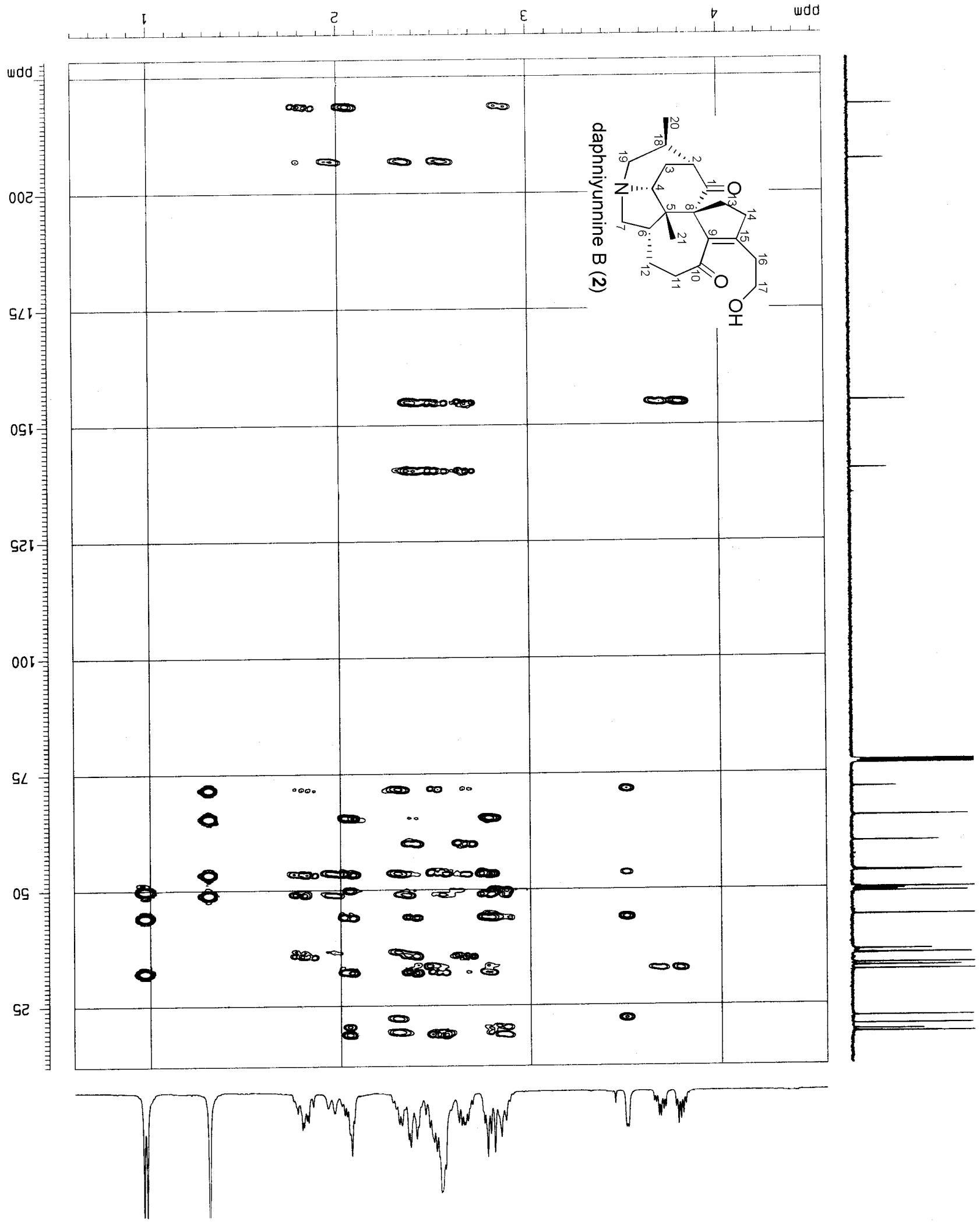


Figure S20. NOESY spectrum of daphniyunnine B (2) in $\mathrm{CDCl}_{3}+\mathrm{CD}_{3} \mathrm{OD}$

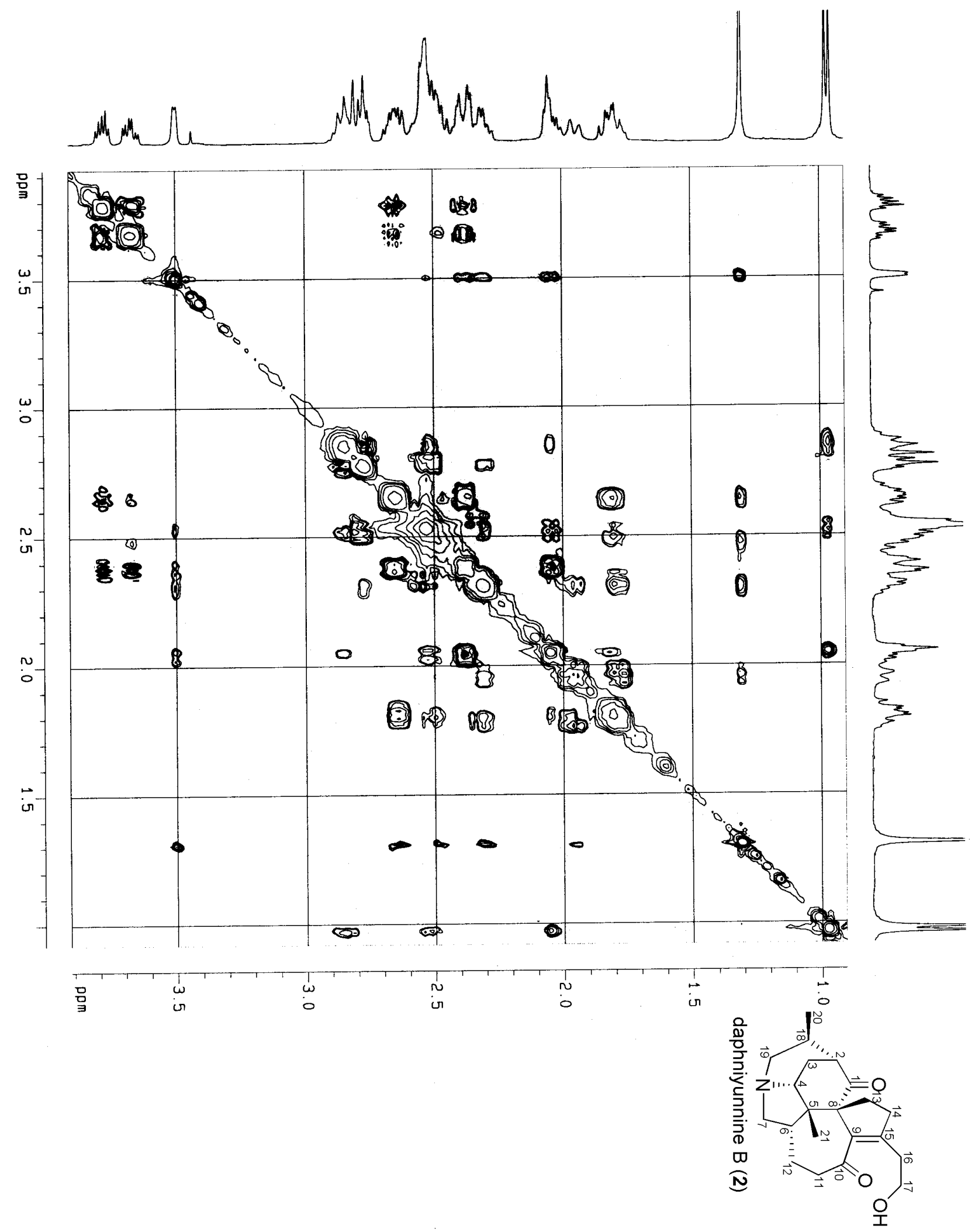


Figure S21. ${ }^{1} \mathrm{H}$ NMR spectrum of daphniyunnine C (3) in $\mathrm{CDCl}_{3}$

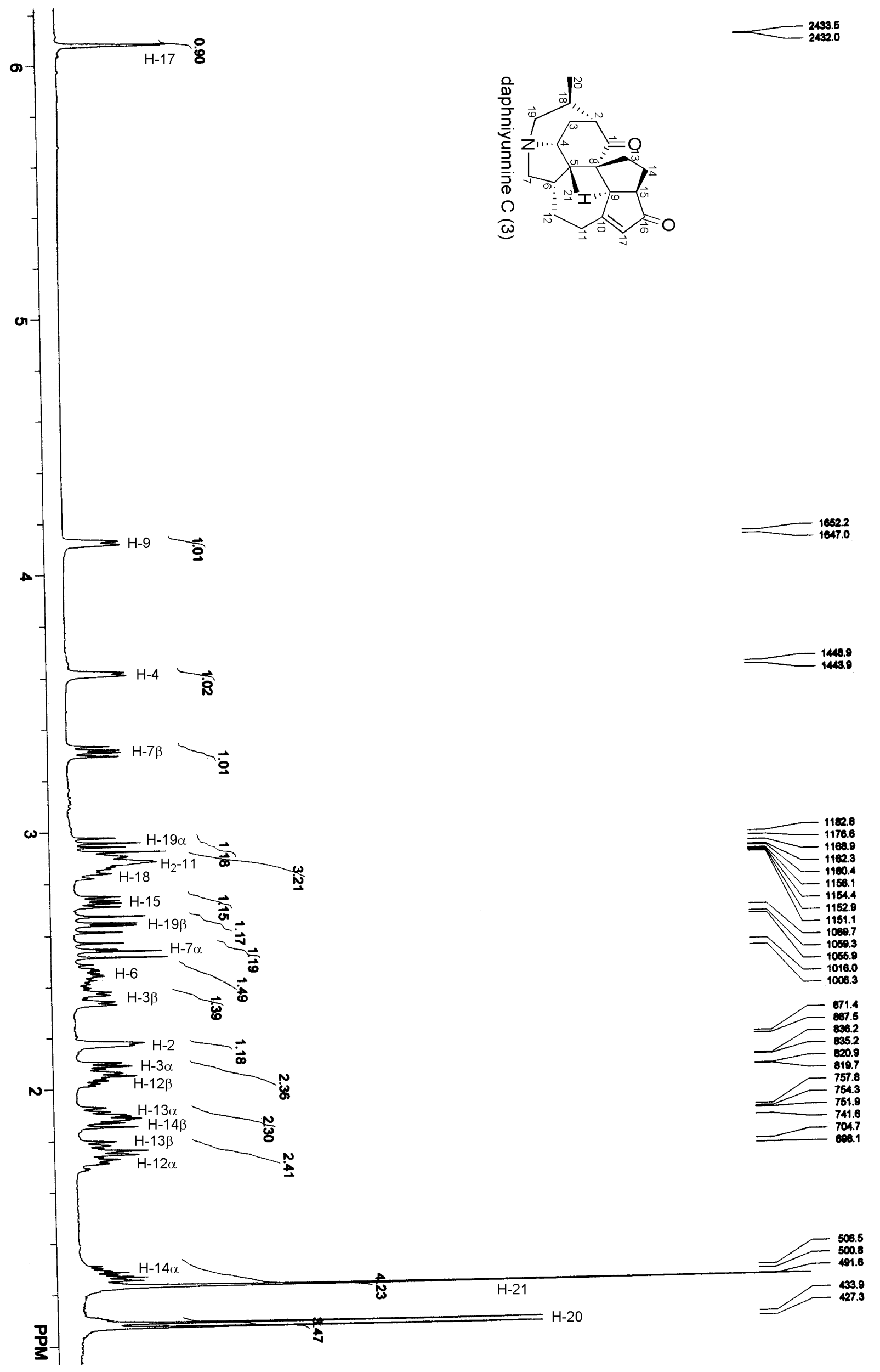


Figure S22. ${ }^{13} \mathrm{C}$ NMR spectrum of daphniyunnine C (3) in $\mathrm{CDCl}_{3}$
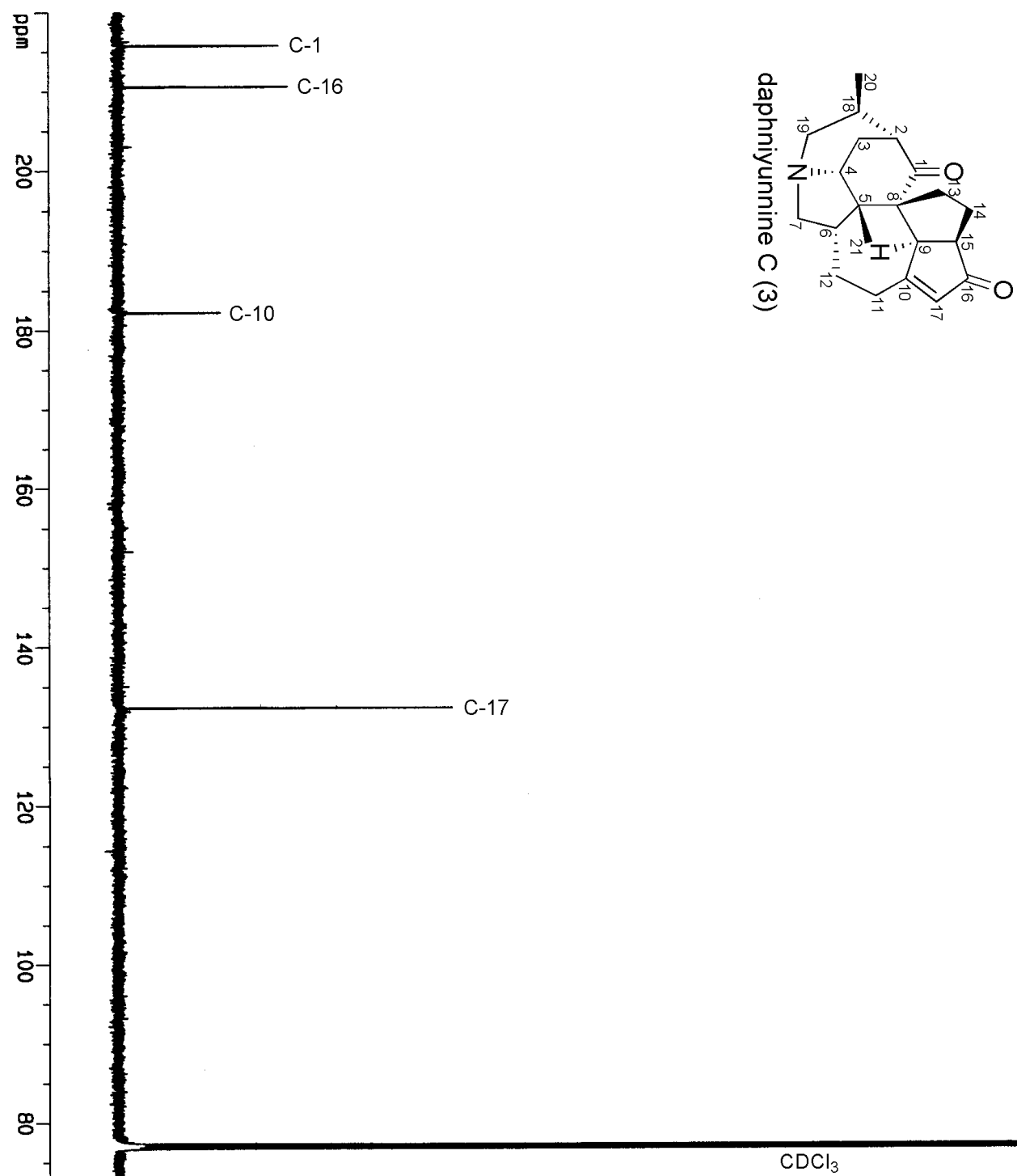

g

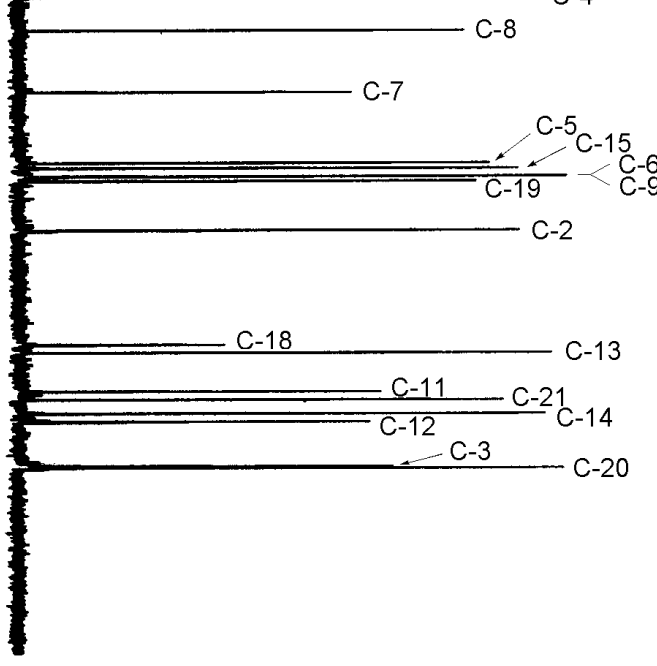

63.739 
Figure S23. Expanded ${ }^{13} \mathrm{C}$ NMR spectrum of daphniyunnine $\mathrm{C}(3)$ in $\mathrm{CDCl}_{3}\left(\delta_{\mathrm{C}} 42-68\right)$
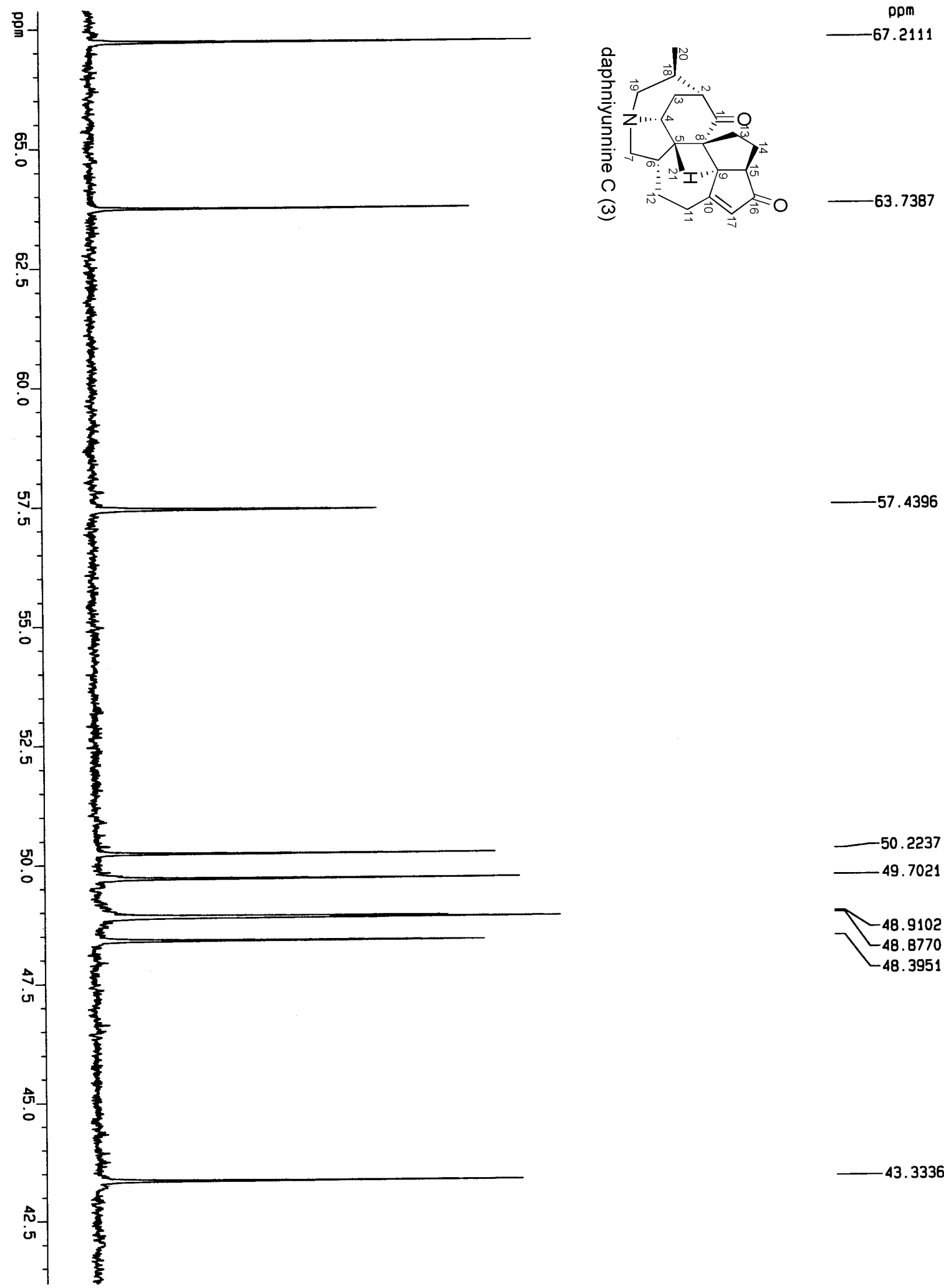

50.2237

49.7021

48.9102

$-48.8770$

48.3951 
Figure S24. DEPT spectrum of daphniyunnine C (3) in $\mathrm{CDCl}_{3}$
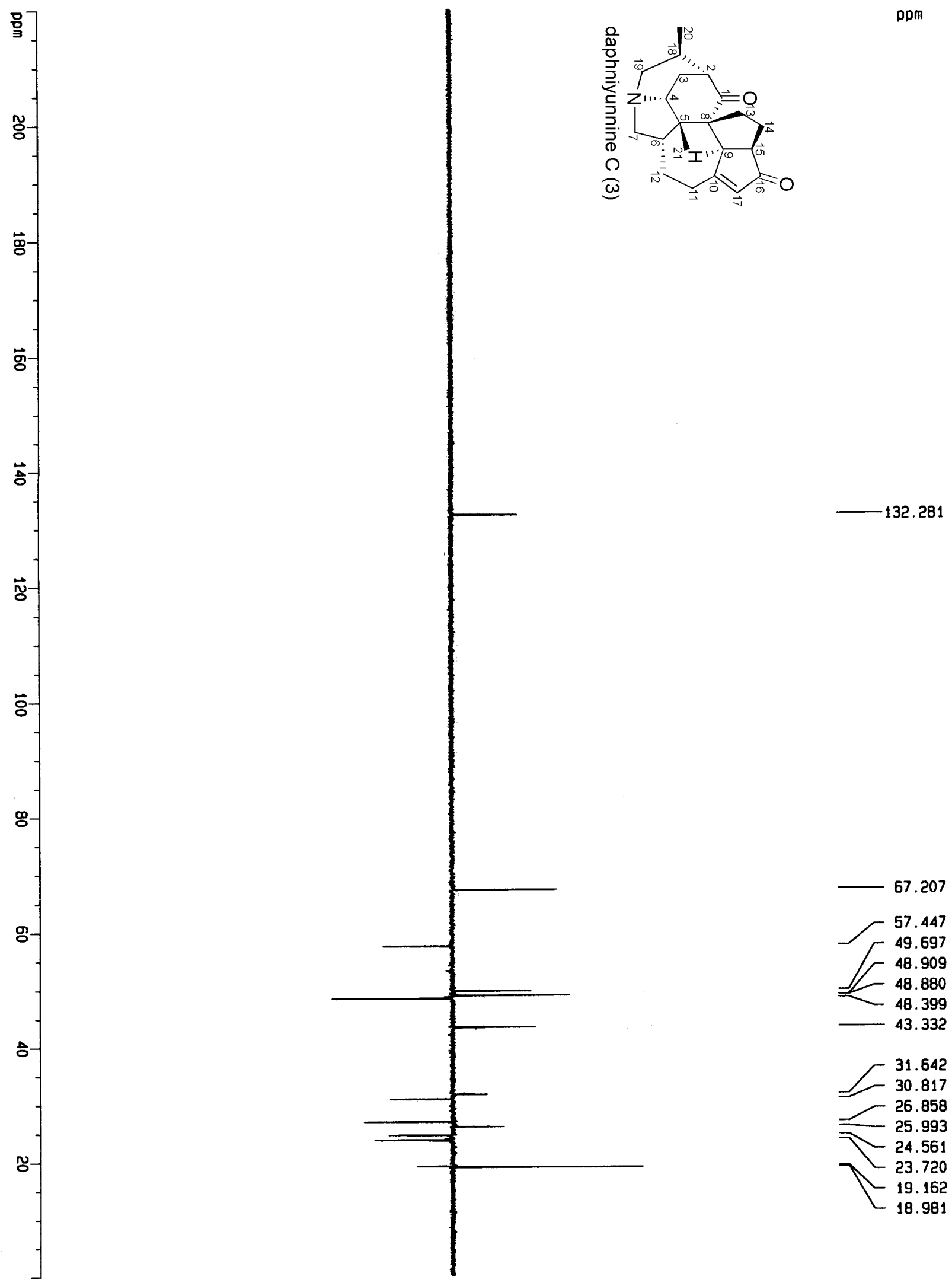

43.332 
Figure S25. Expanded DEPT spectrum of daphniyunnine C in (3) $\mathrm{CDCl}_{3}\left(\delta_{\mathrm{C}} 42-68\right)$
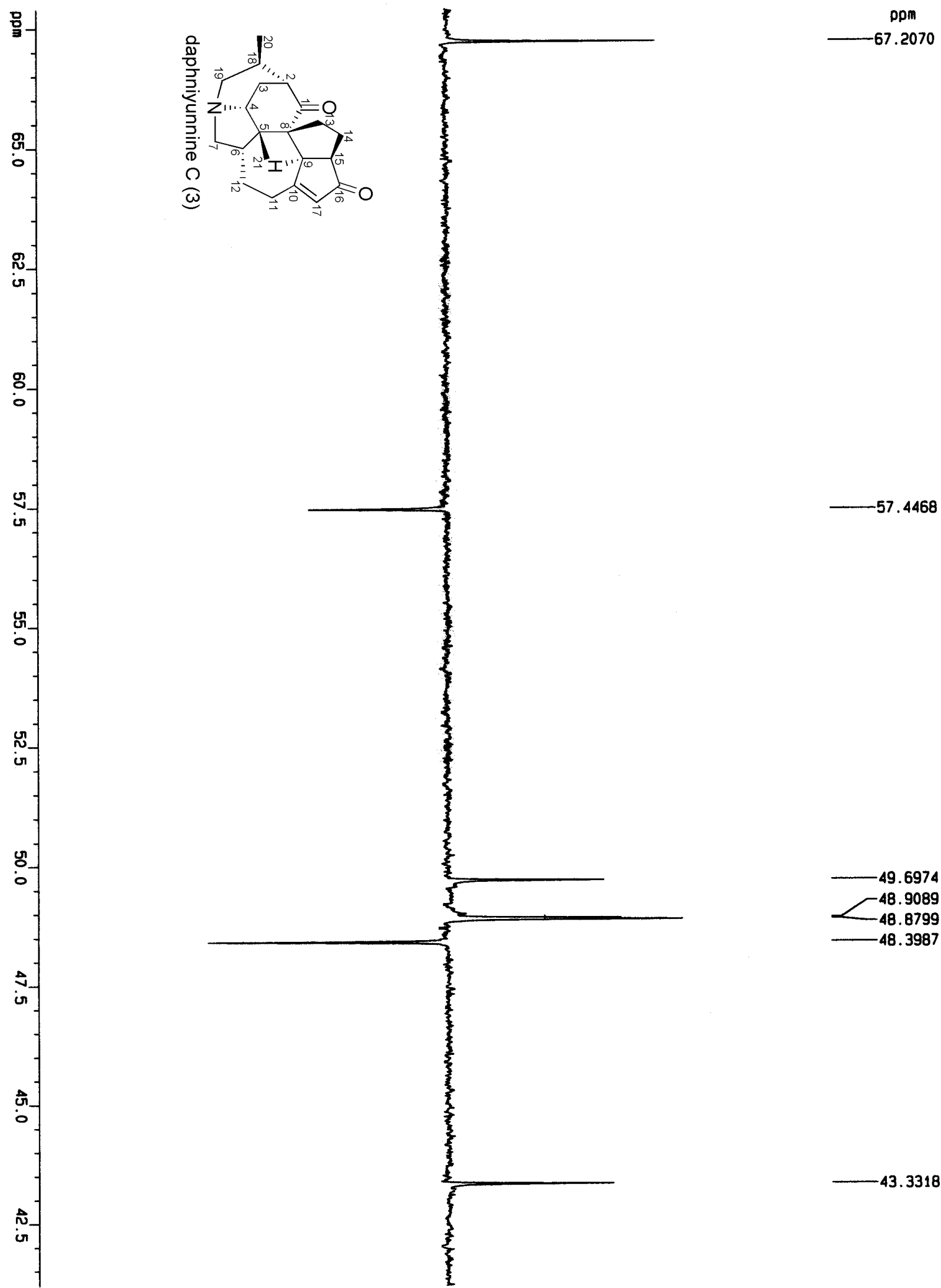

$-43.3318$ 
Figure S26. EIMS spectrum of daphniyunnine C (3)

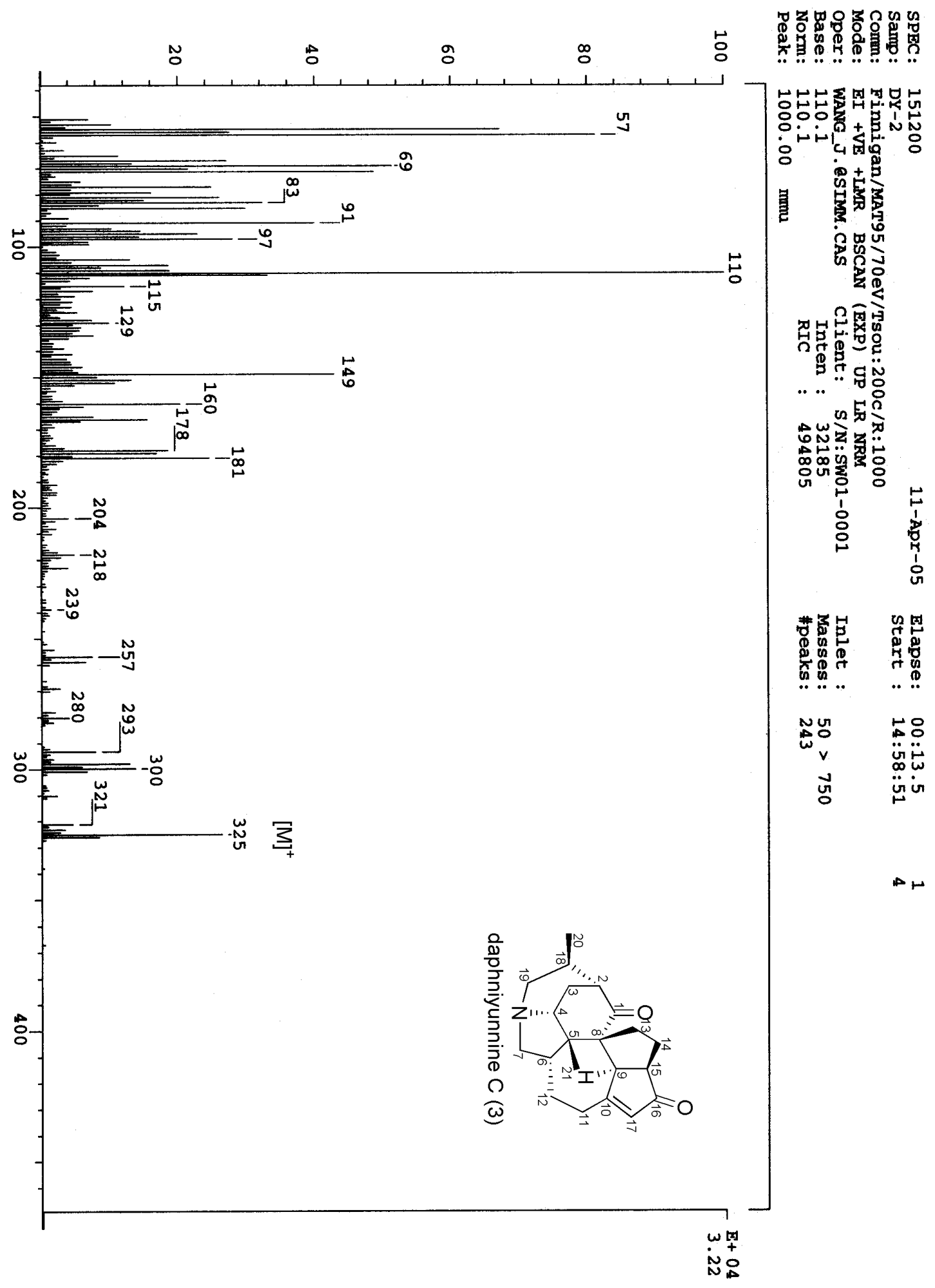


Figure S27. IR spectrum of daphniyunnine C (3)

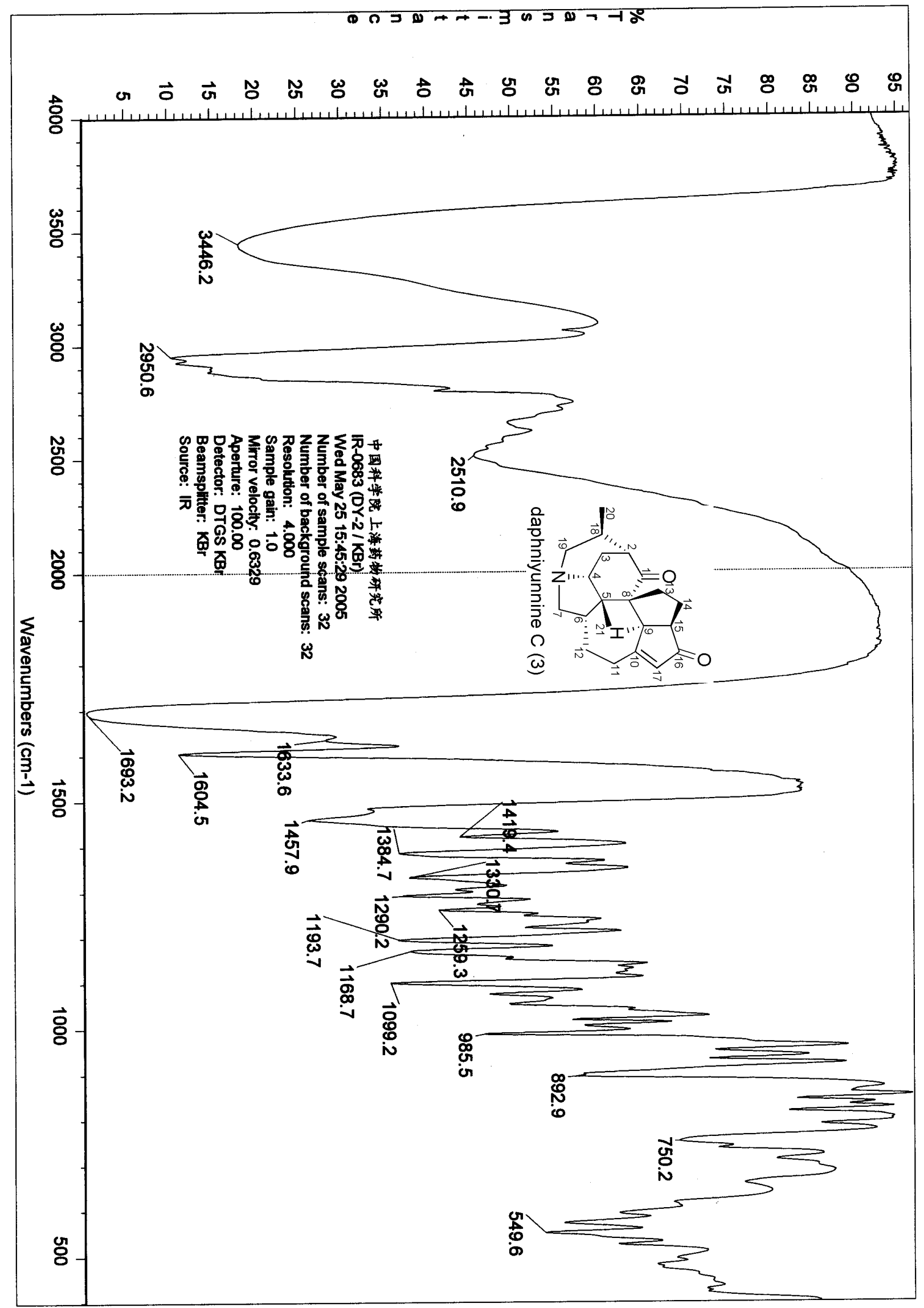


Figure S28. ${ }^{1} \mathrm{H}-{ }^{1} \mathrm{H}$ COSY spectrum of daphniyunnine $\mathrm{C}(3)$ in $\mathrm{CDCl}_{3}$
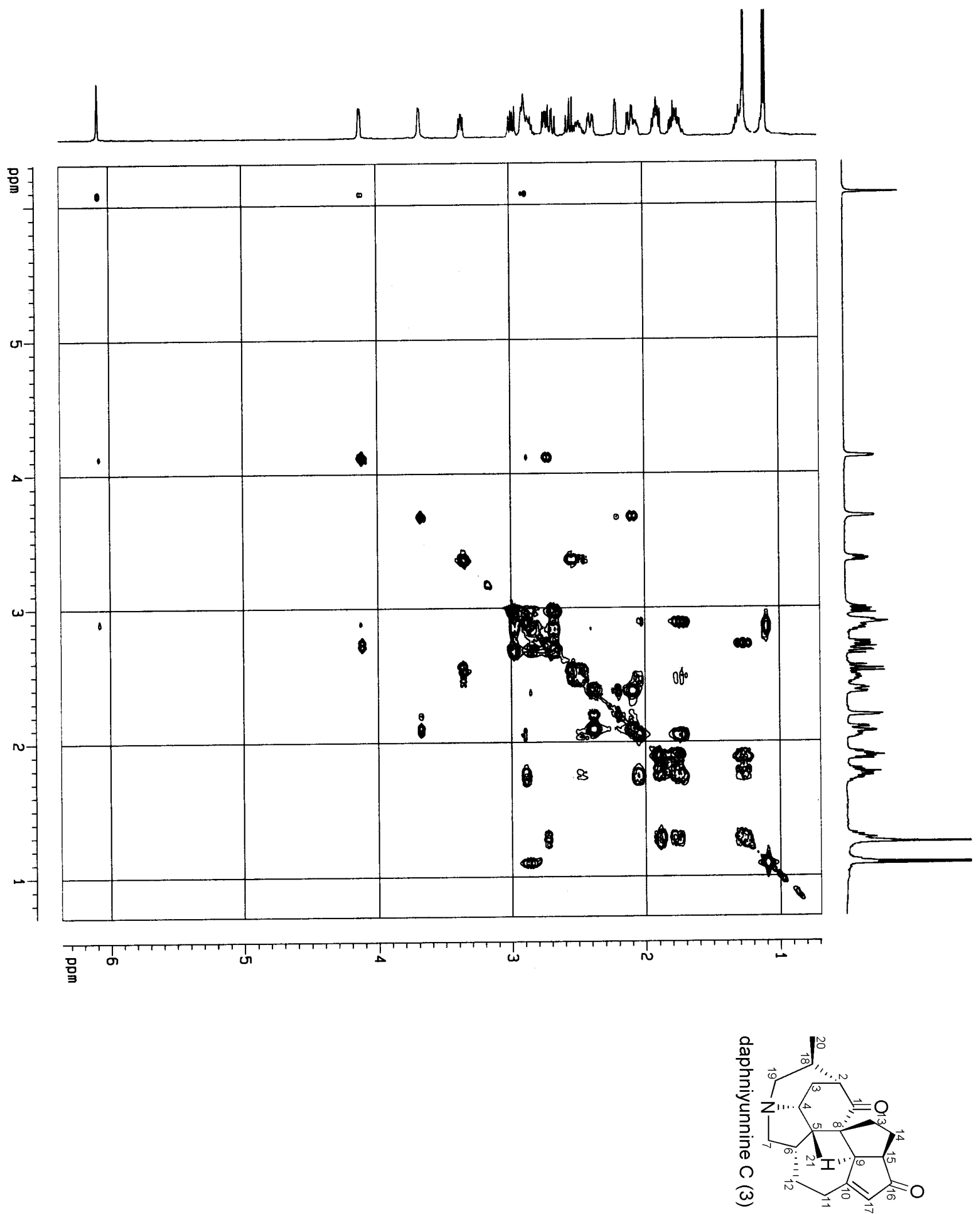
Figure S29. Expanded ${ }^{1} \mathrm{H}-{ }^{1} \mathrm{H}$ COSY spectrum of daphniyunnine C (3) in $\mathrm{CDCl}_{3}\left(\delta_{\mathrm{H}} 0.8-4.3\right)$

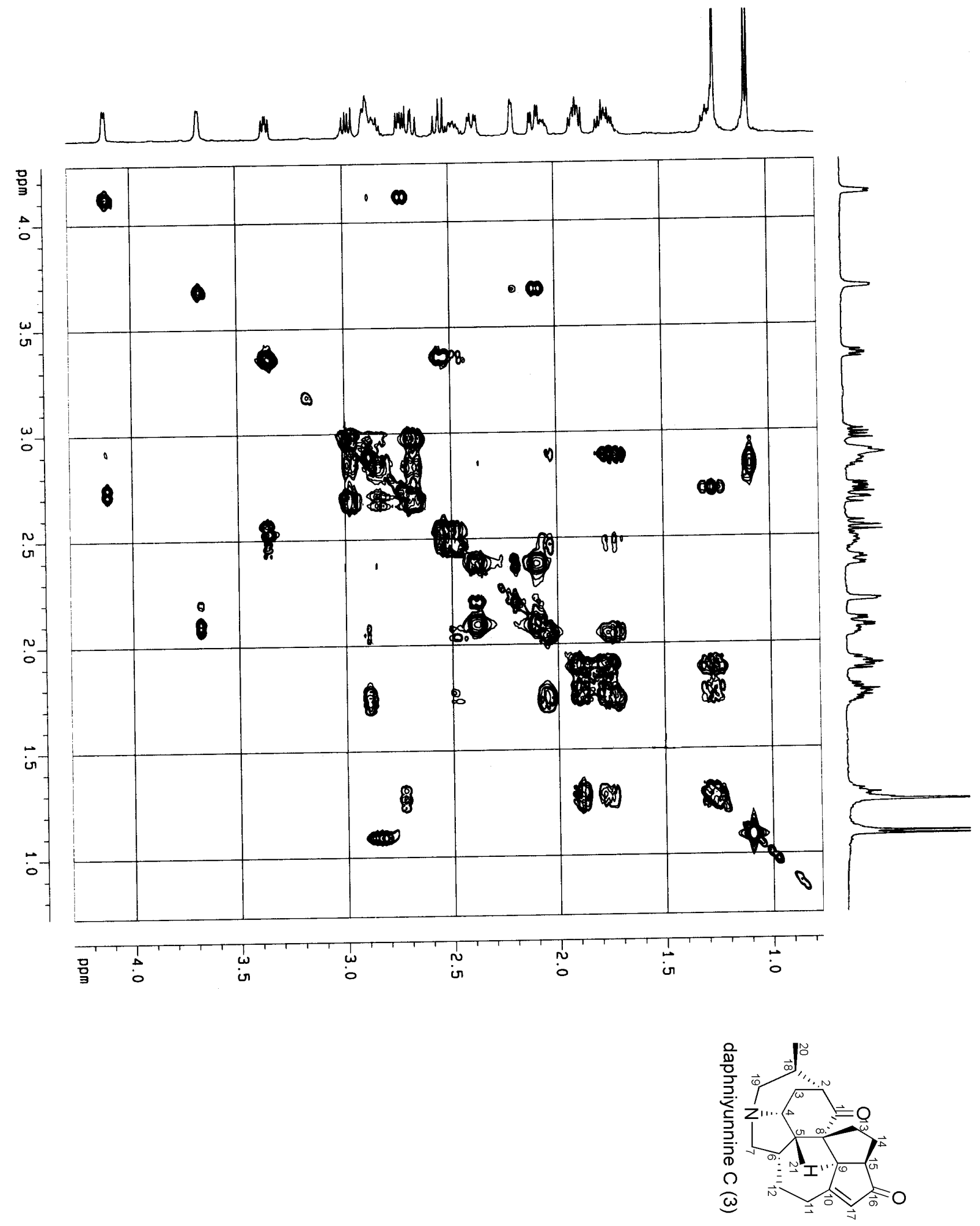


Figure S30. HMQC spectrum of daphniyunnine C (3) in $\mathrm{CDCl}_{3}$

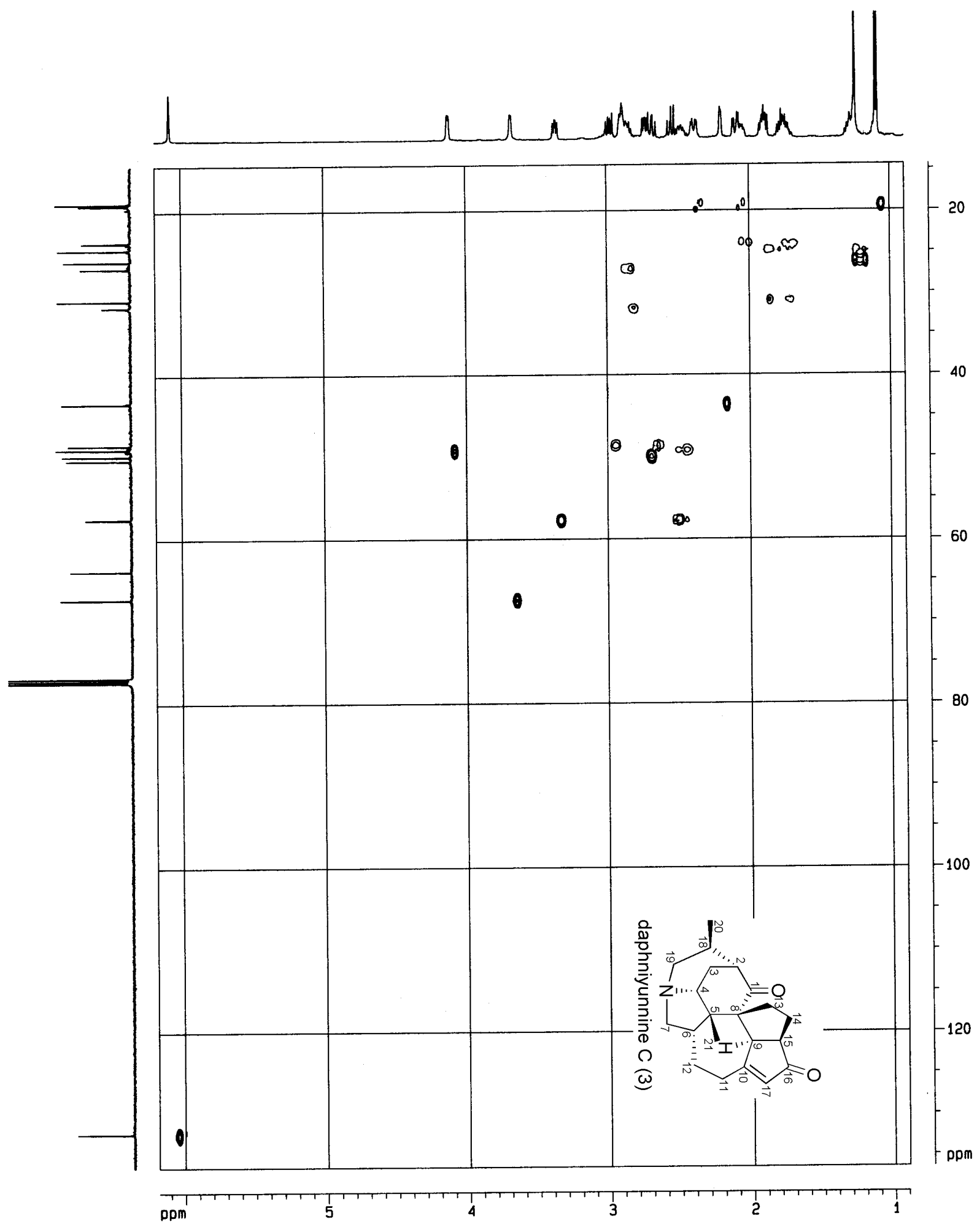


Figure S31. HMBC spectrum of daphniyunnine C (3) in $\mathrm{CDCl}_{3}$

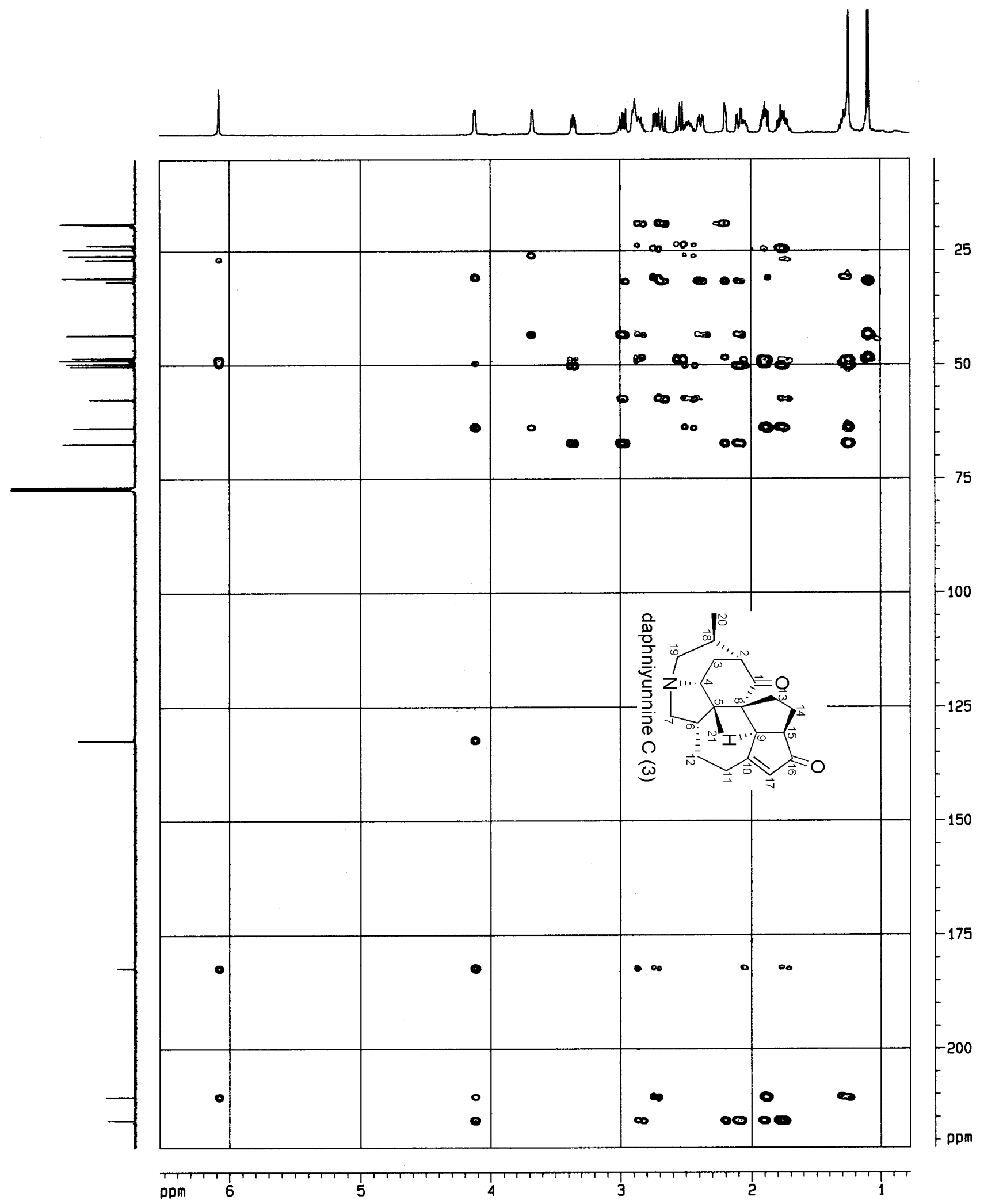


Figure S32. NOESY spectrum of daphniyunnine C (3) in $\mathrm{CDCl}_{3}$
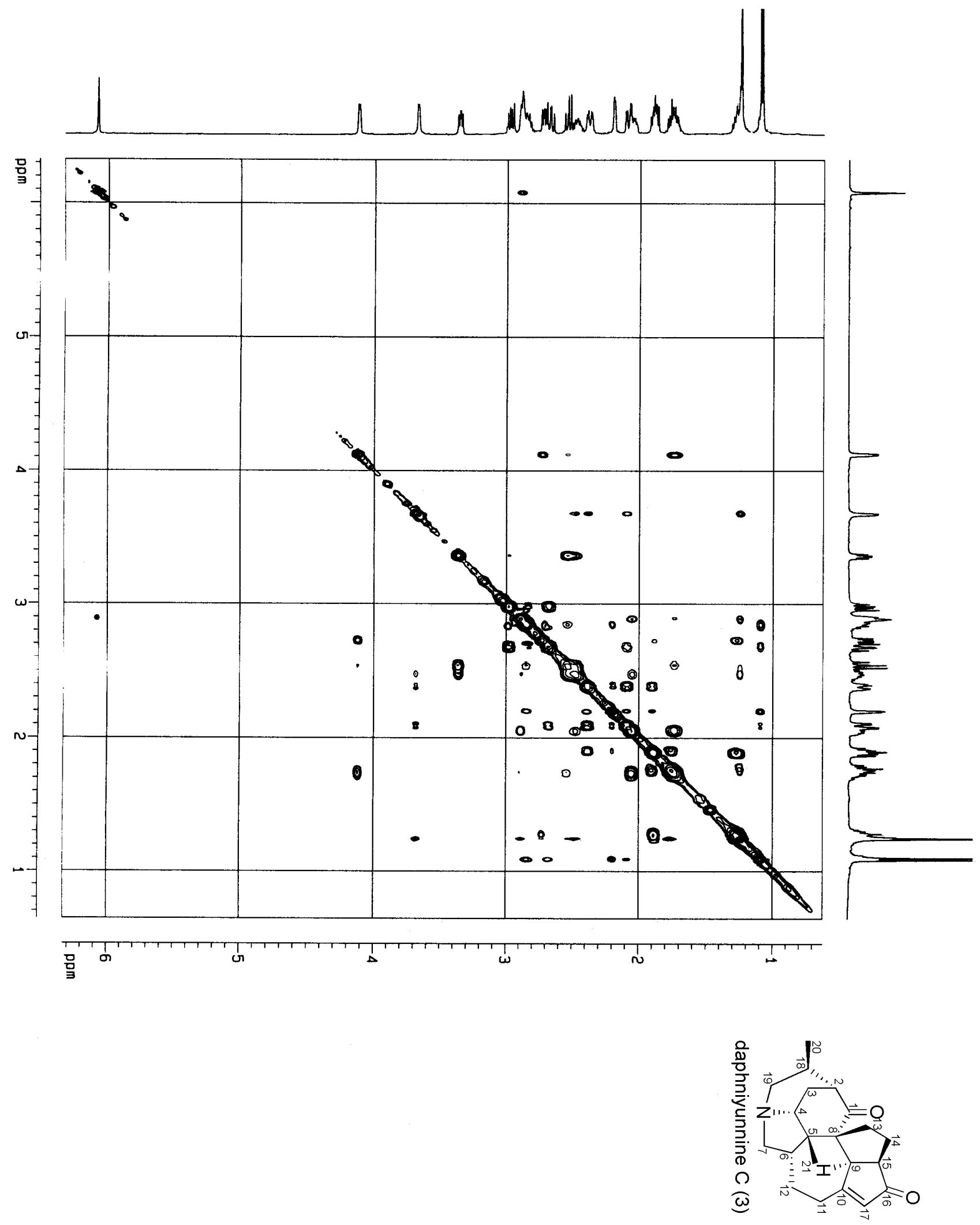

35 
Figure S33. Expanded NOESY spectrum of daphniyunnine C (3) in $\mathrm{CDCl}_{3}\left(\delta_{\mathrm{H}} 0.9-4.3\right)$
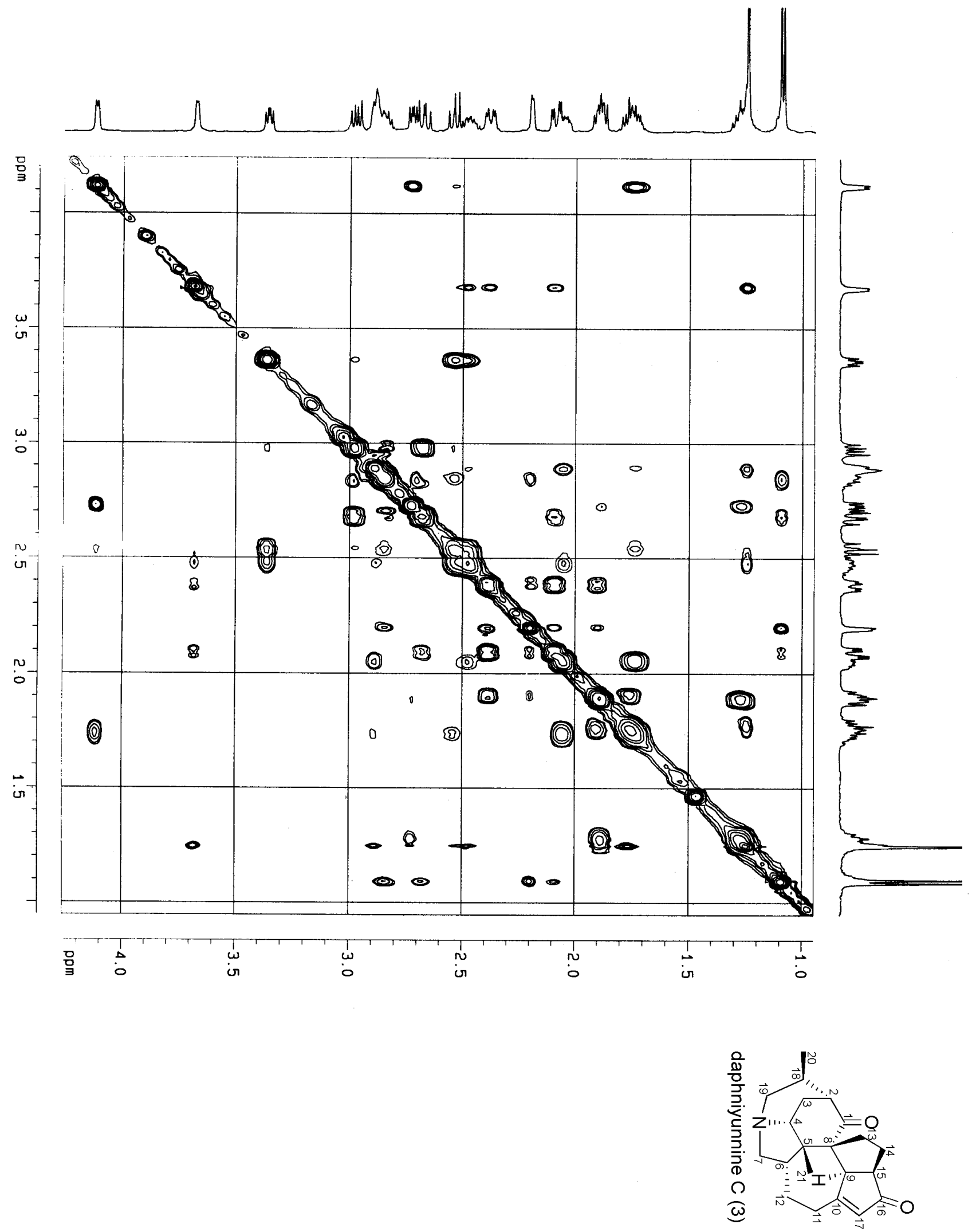
Figure S34. ${ }^{1} \mathrm{H}$ NMR spectrum of daphniyunnine D (4) in $\mathrm{CDCl}_{3}$
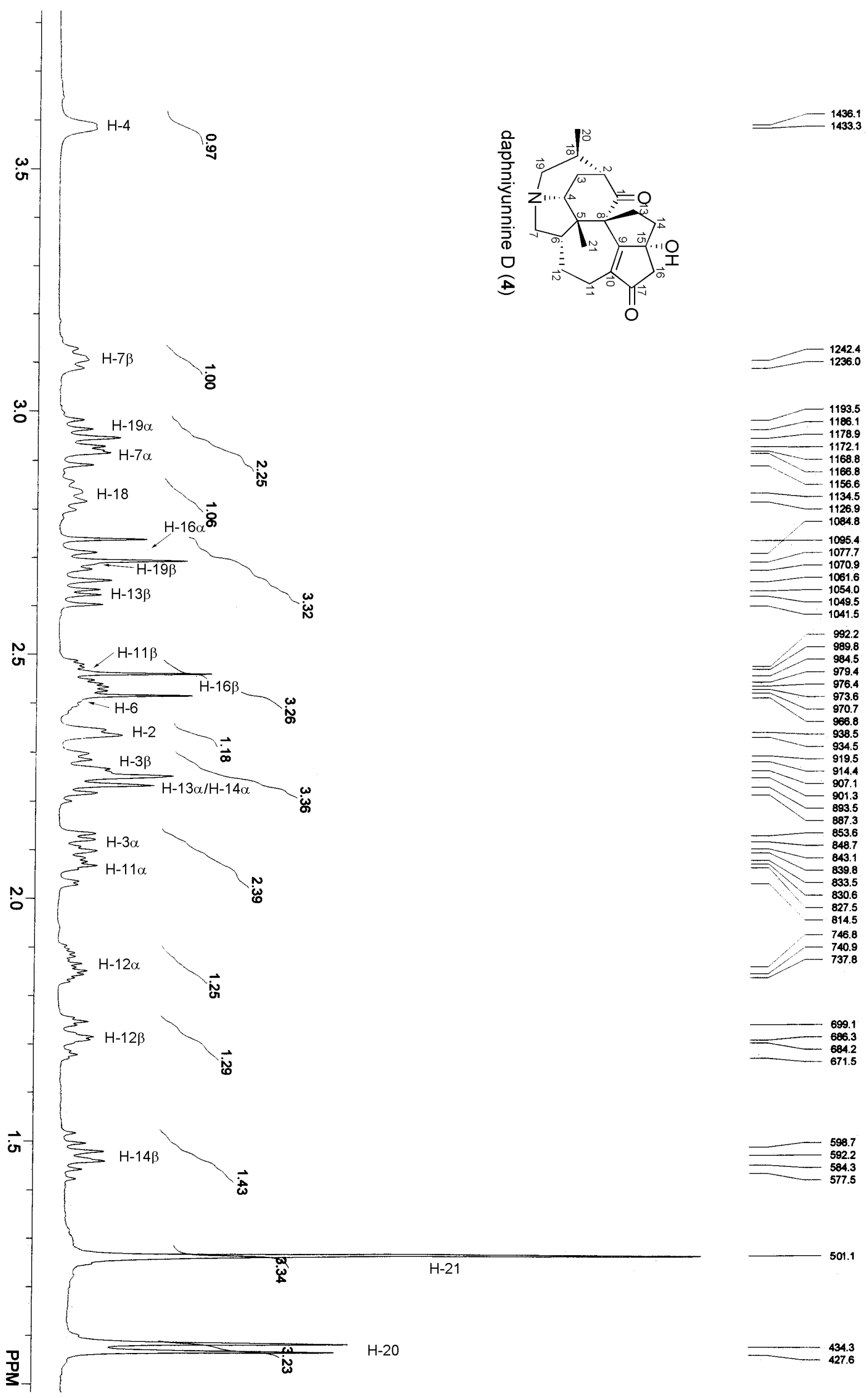
Figure S35. ${ }^{13} \mathrm{C}$ NMR spectrum of daphniyunnine D (4) in $\mathrm{CDCl}_{3}$

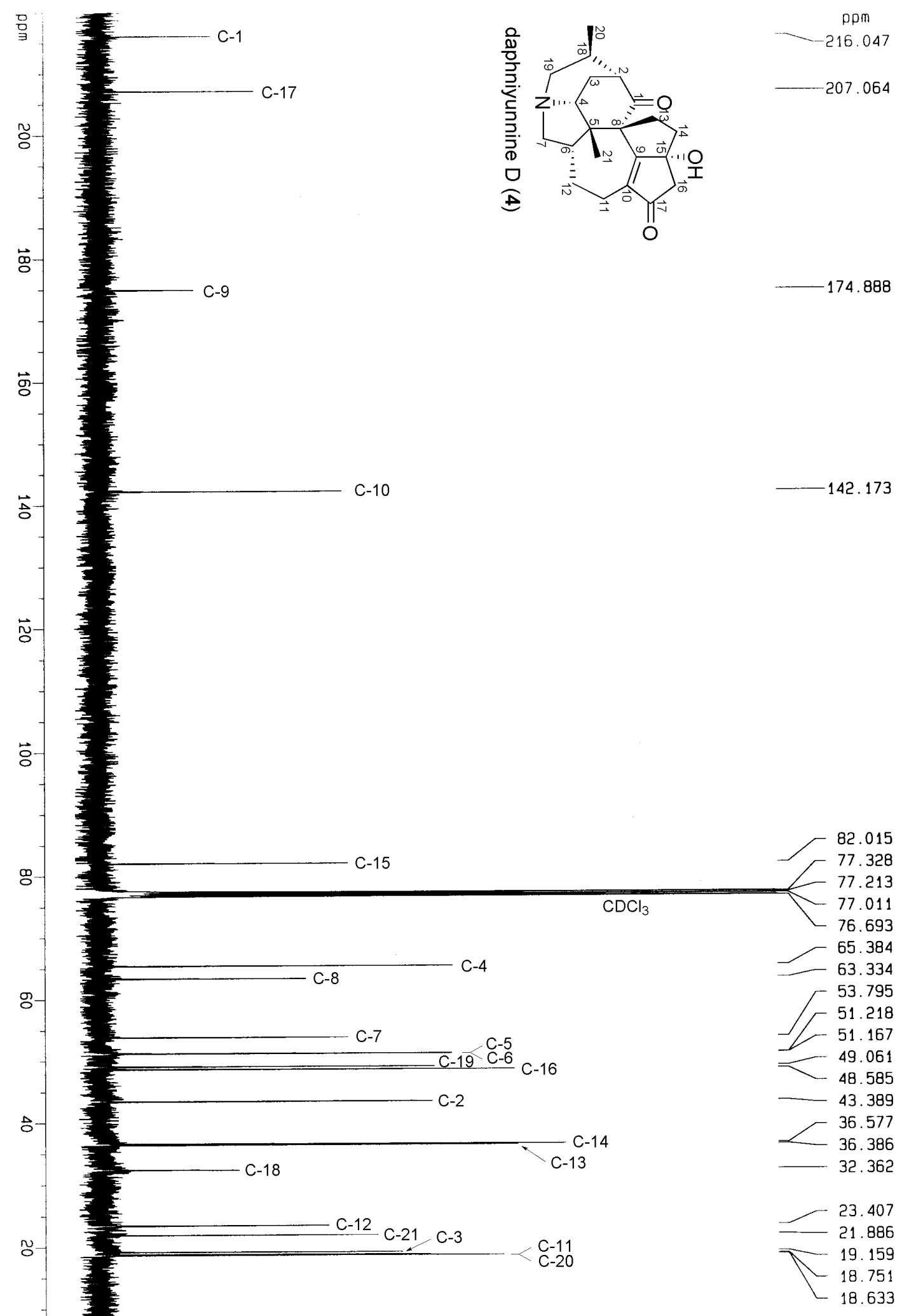


Figure S36. Expanded ${ }^{13} \mathrm{C}$ NMR spectrum of daphniyunnine D (4) in $\mathrm{CDCl}_{3}\left(\delta_{\mathrm{C}} 13-66\right)$
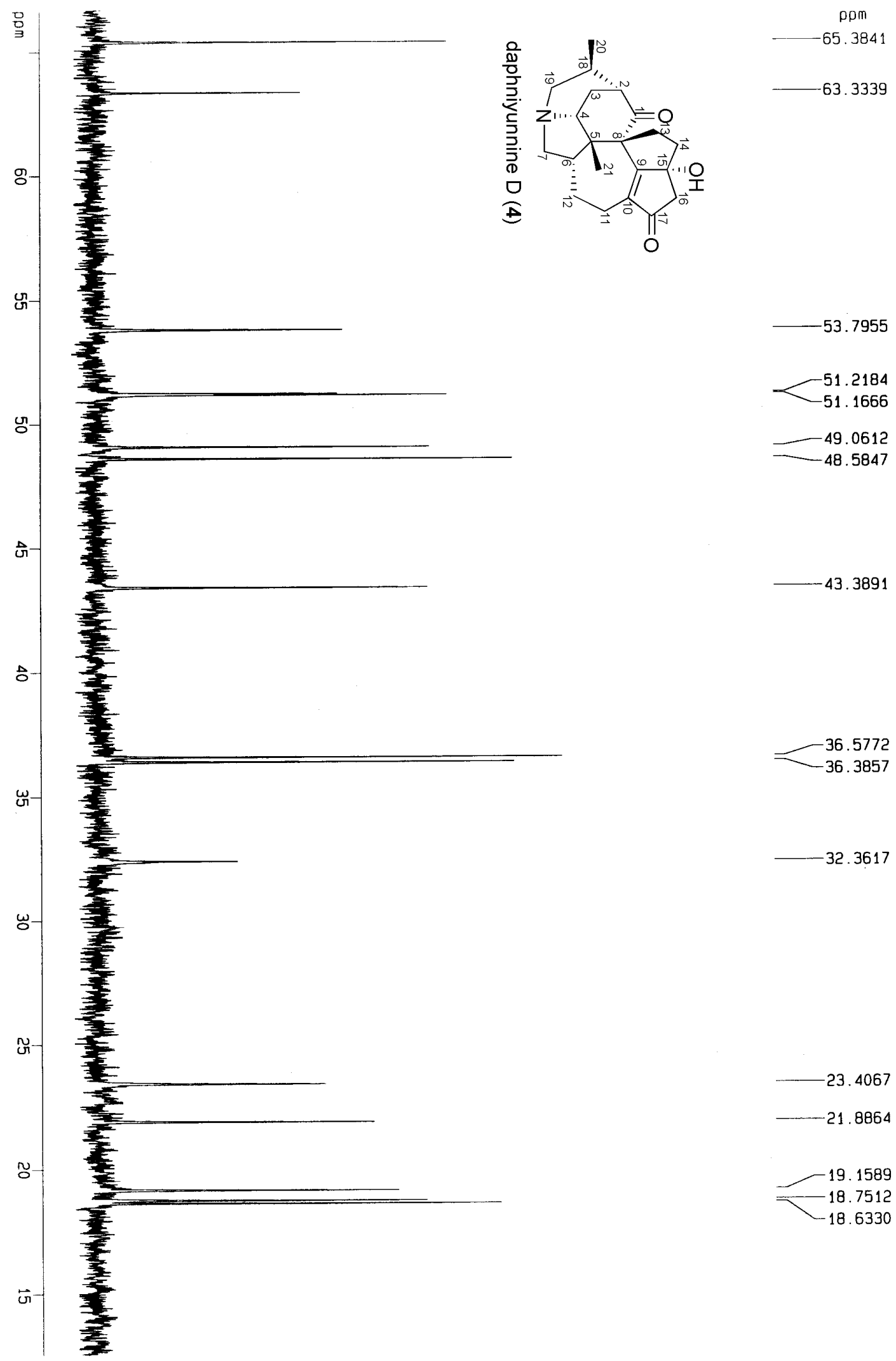

43. 3891

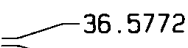

$-36.3857$

32.3617

23. 4067

21.8864

19. 1589

$-18.7512$

$-18.6330$ 
Figure S37. DEPT spectrum of daphniyunnine D (4) in $\mathrm{CDCl}_{3}$

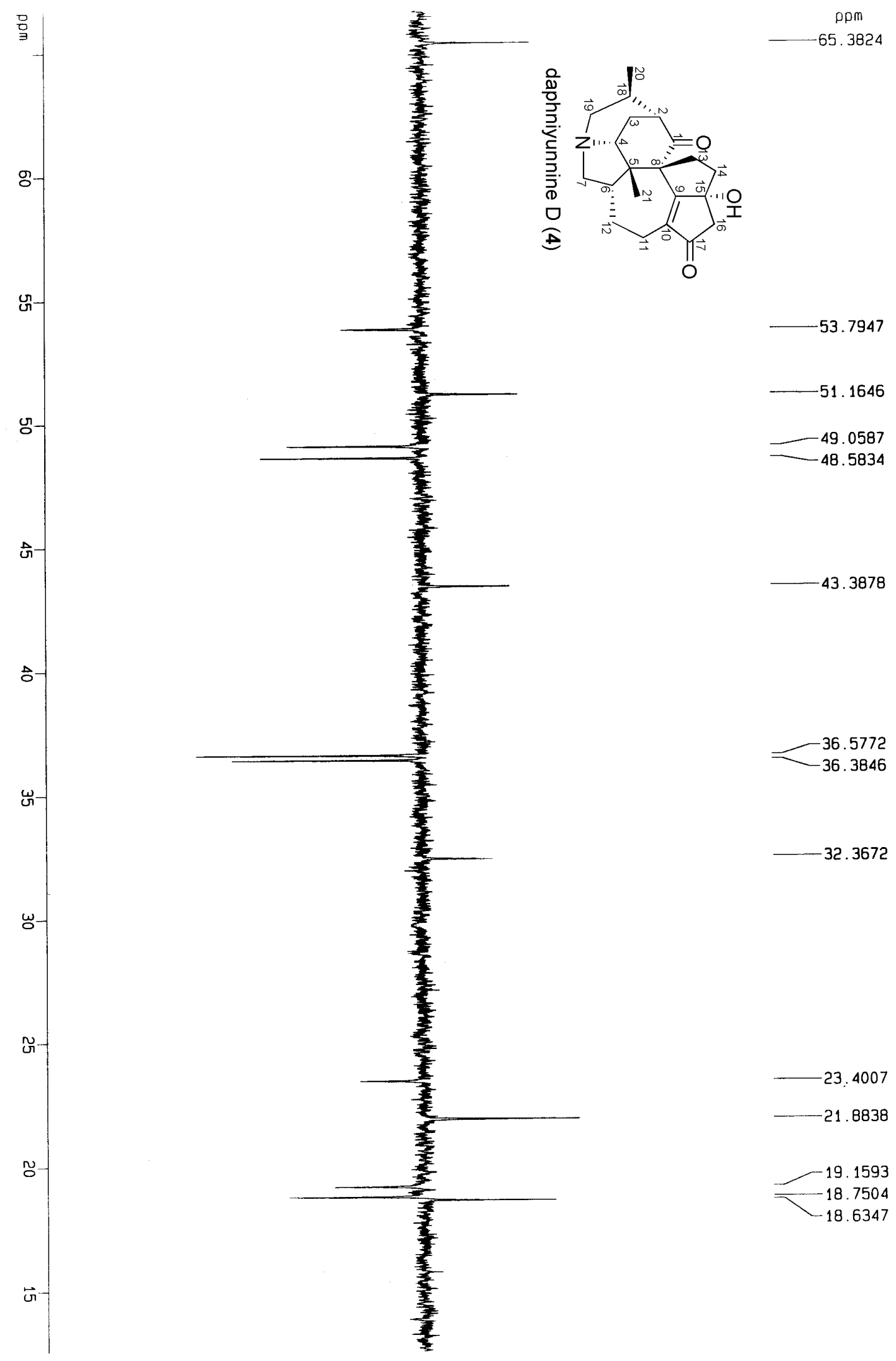


Figure S38. EIMS spectrum of daphniyunnine D (4)

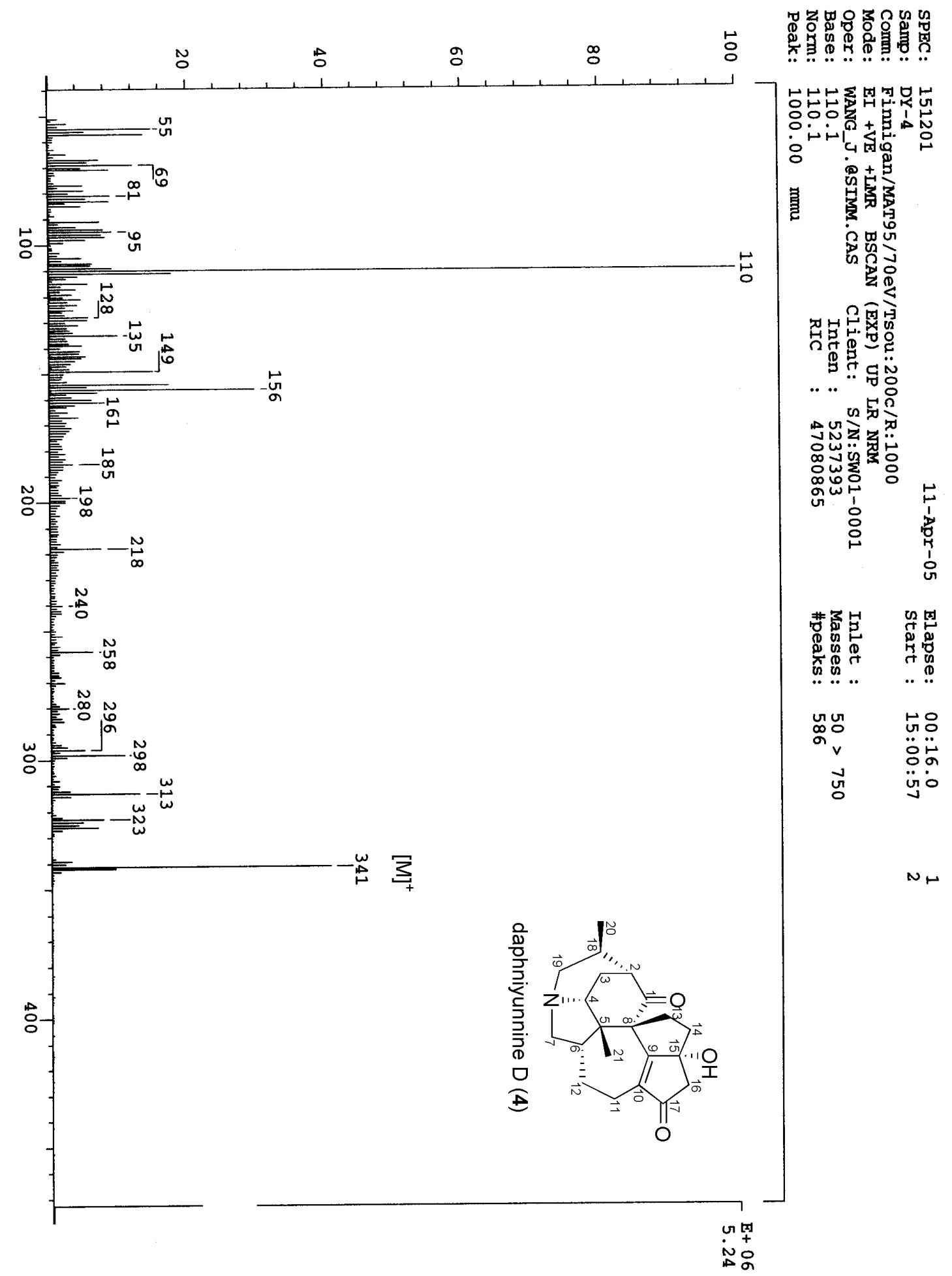


Figure S39. IR spectrum of daphniyunnine D (4)

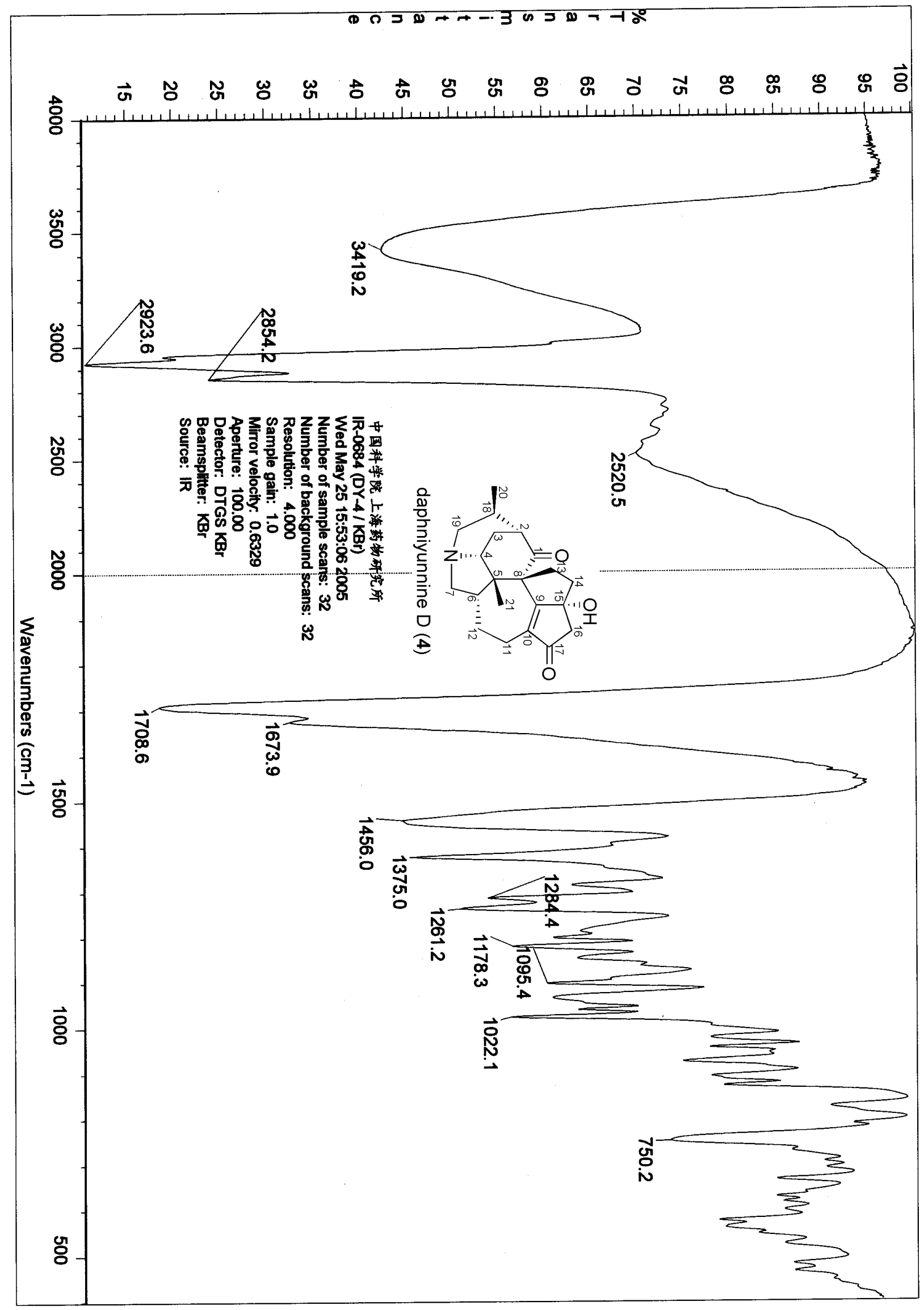


Figure S40. ${ }^{1} \mathrm{H}-{ }^{1} \mathrm{H}$ COSY spectrum of daphniyunnine D (4) in $\mathrm{CDCl}_{3}$
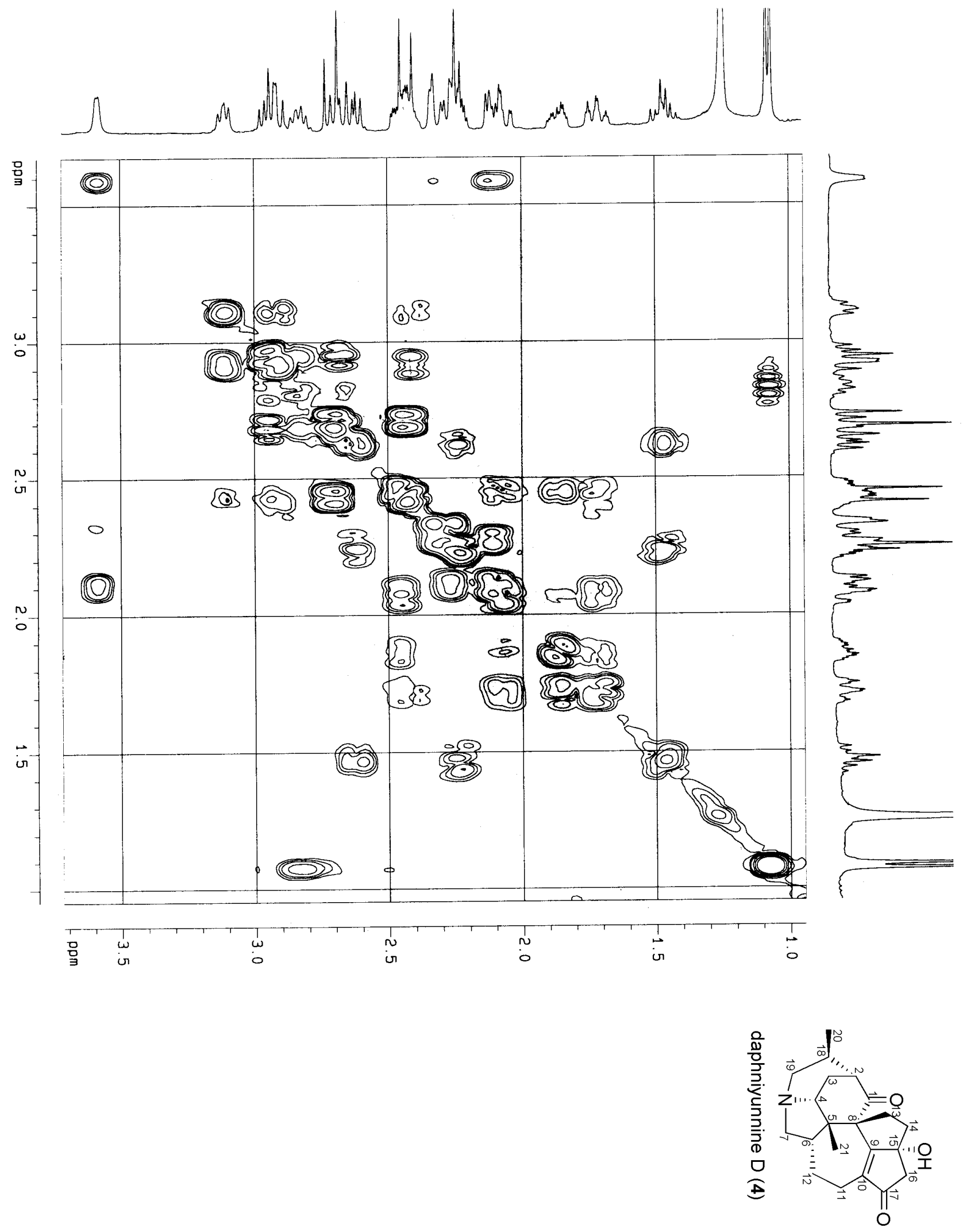
Figure S41. HMQC spectrum of daphniyunnine D (4) in $\mathrm{CDCl}_{3}$

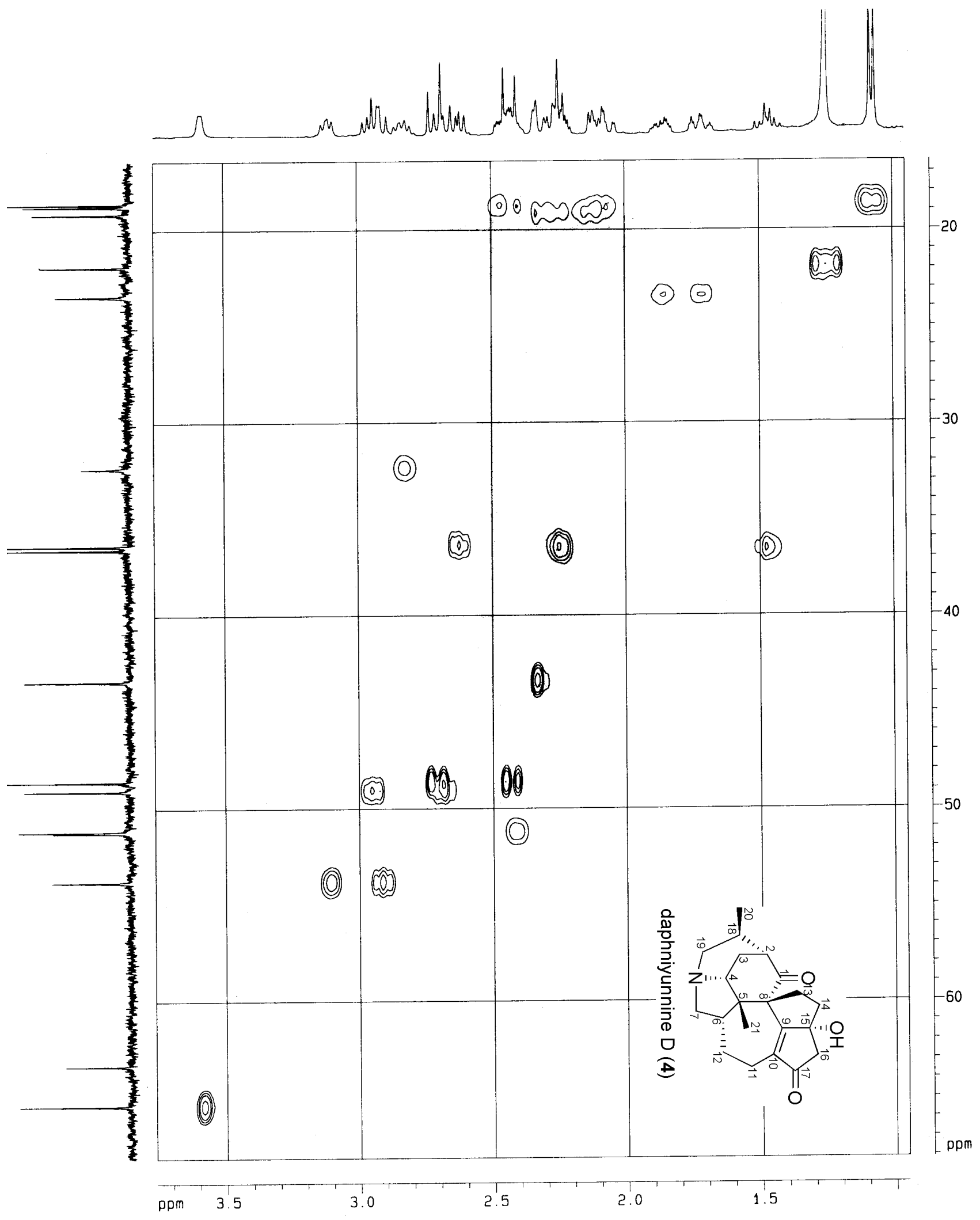


Figure S42. HMBC spectrum of daphniyunnine D (4) in $\mathrm{CDCl}_{3}$

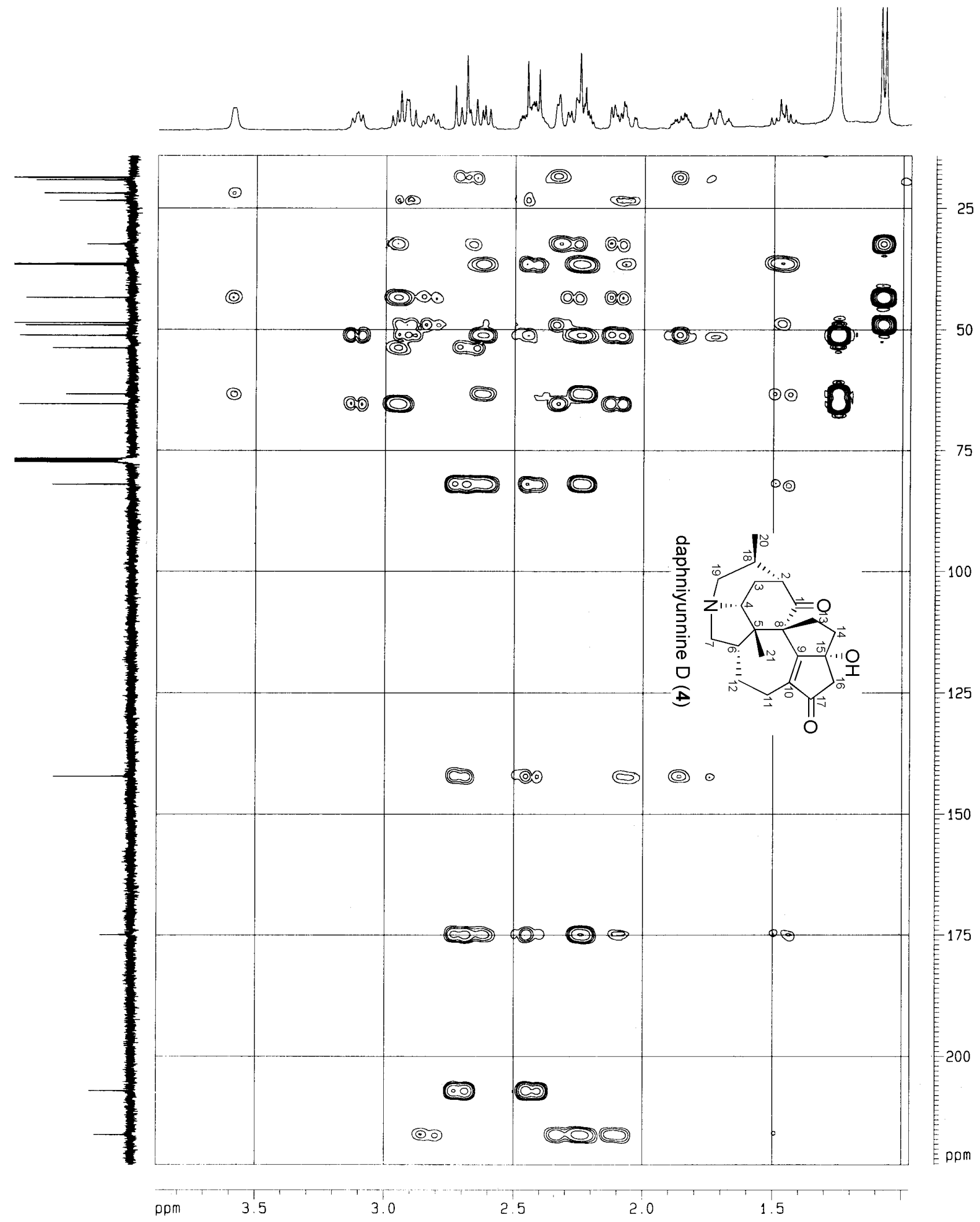


Figure S43. NOESY spectrum of daphniyunnine D (4) in $\mathrm{CDCl}_{3}$

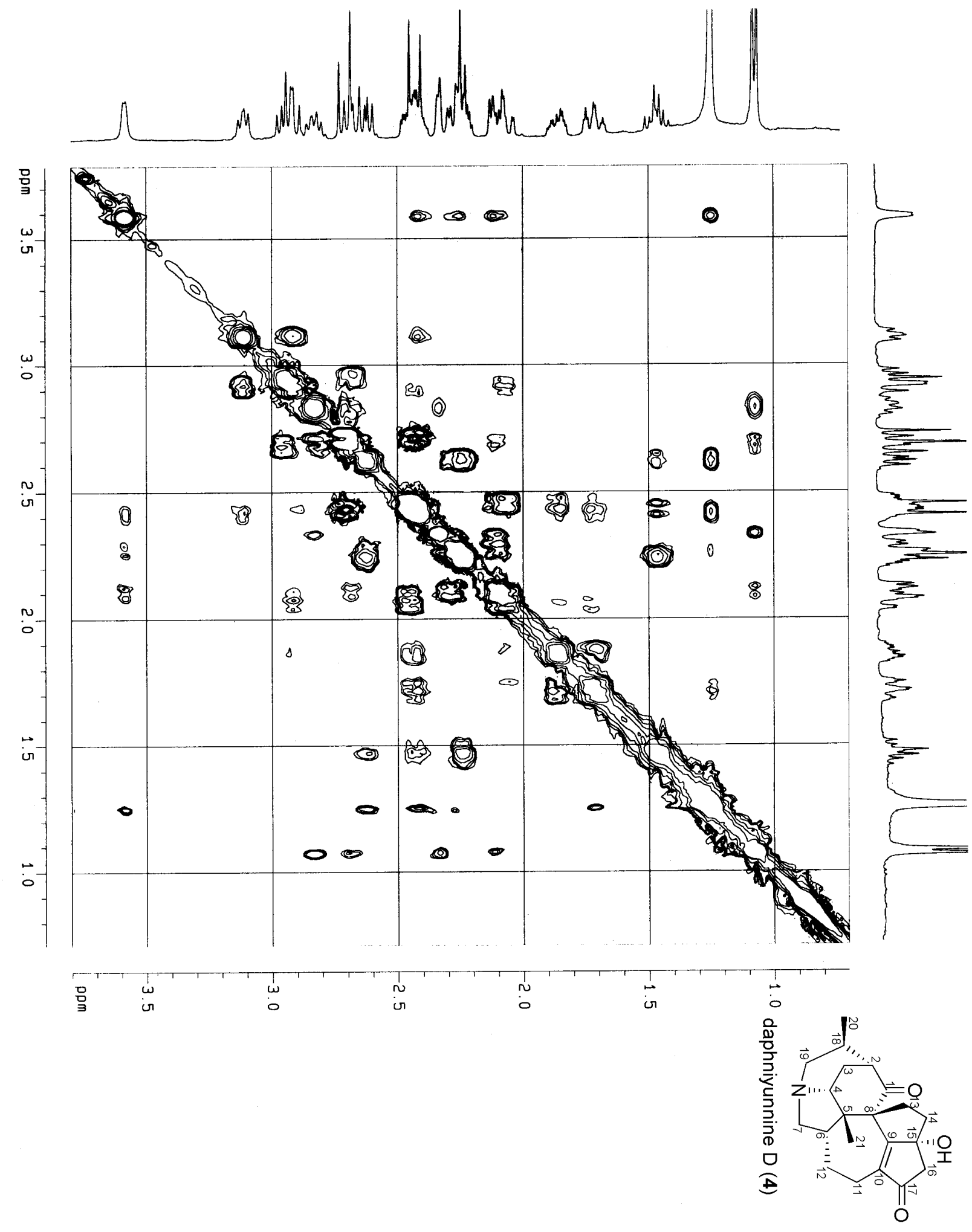


Figure S44. ${ }^{1} \mathrm{H}$ NMR spectrum of daphniyunnine $\mathrm{E}(5)$ in $\mathrm{CDCl}_{3}$

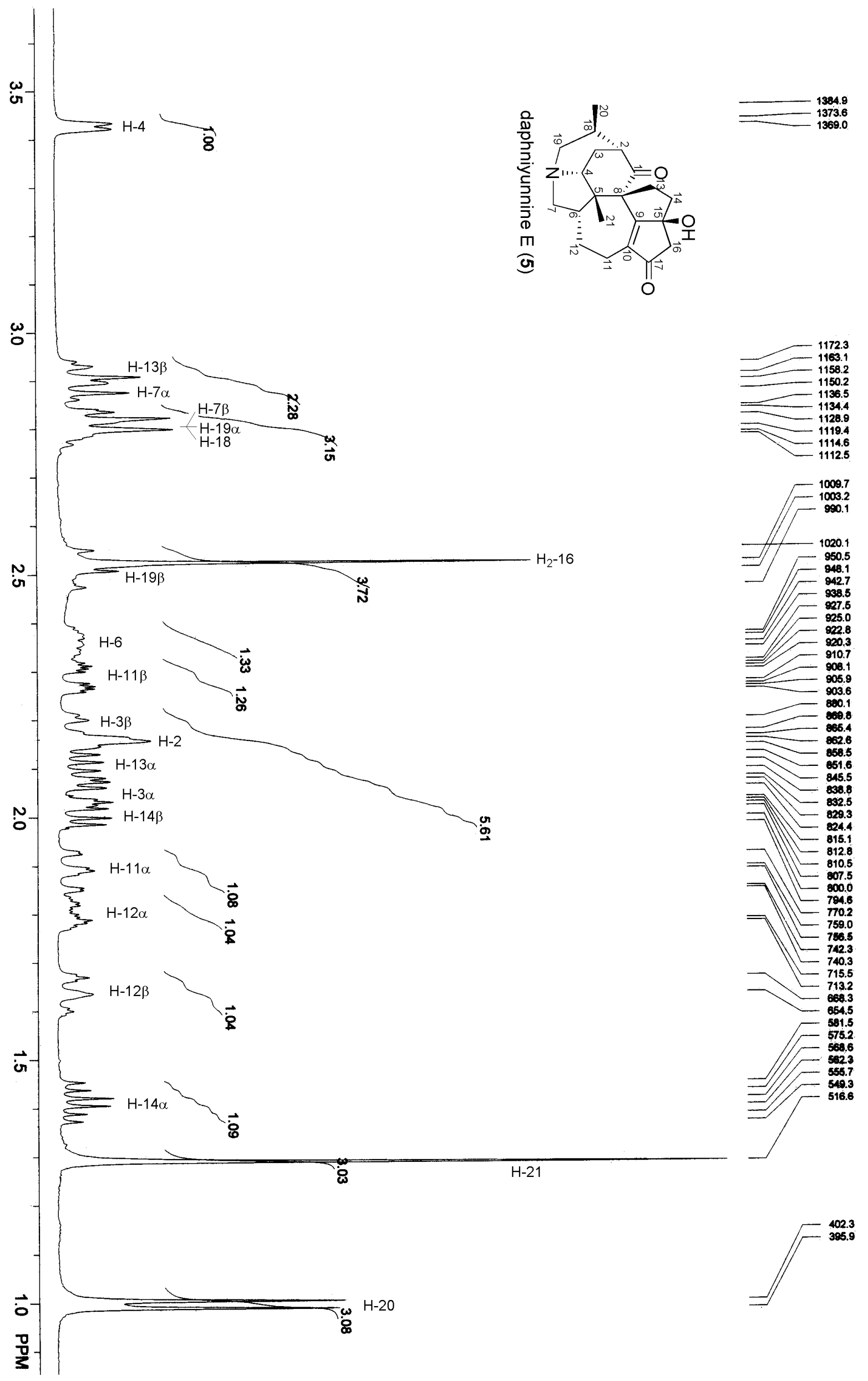


Figure S45. ${ }^{13} \mathrm{C}$ NMR spectrum of daphniyunnine $\mathrm{E}(5)$ in $\mathrm{CDCl}_{3}$

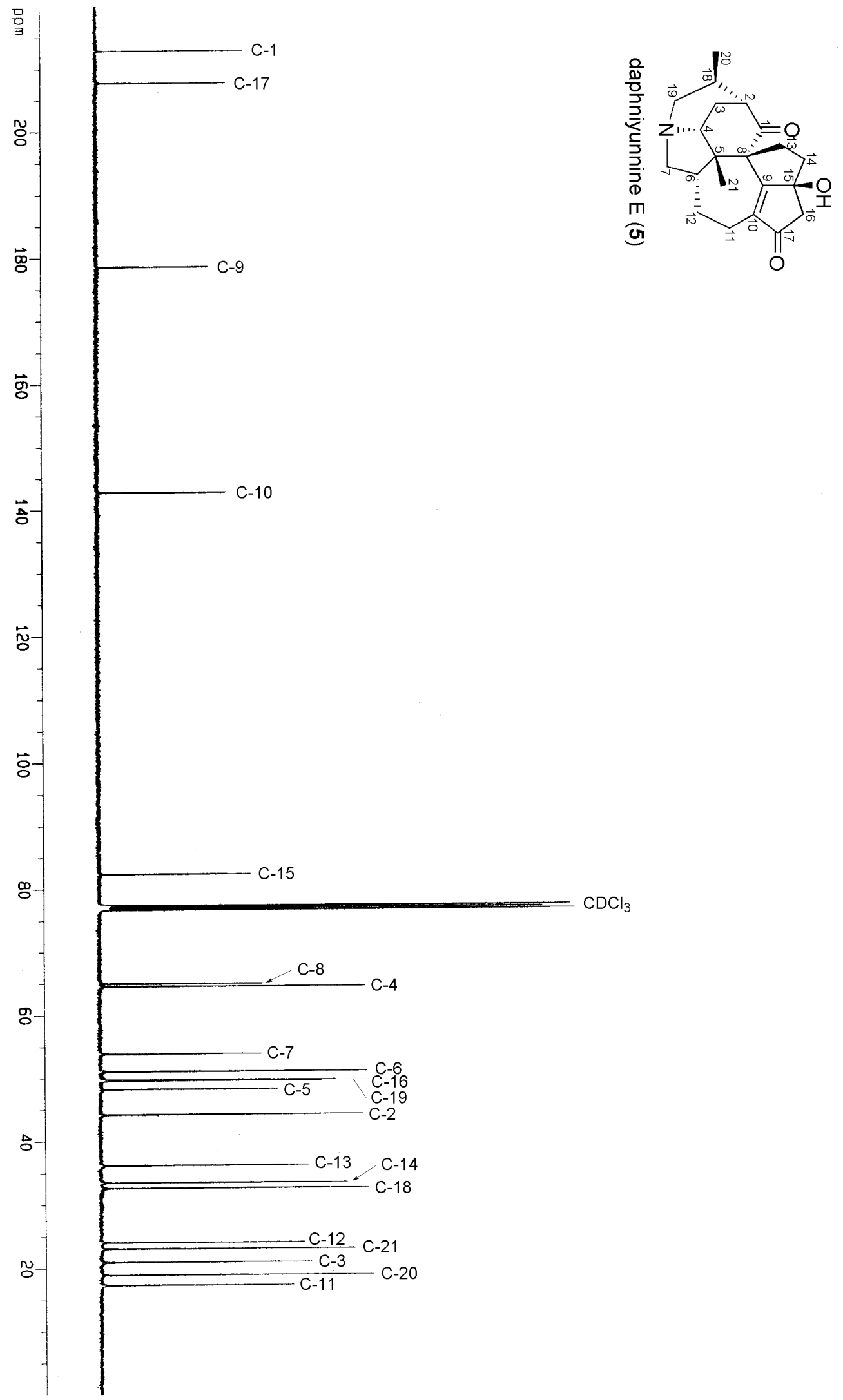

ppm

$-212.830$

207.695

$-178.570$
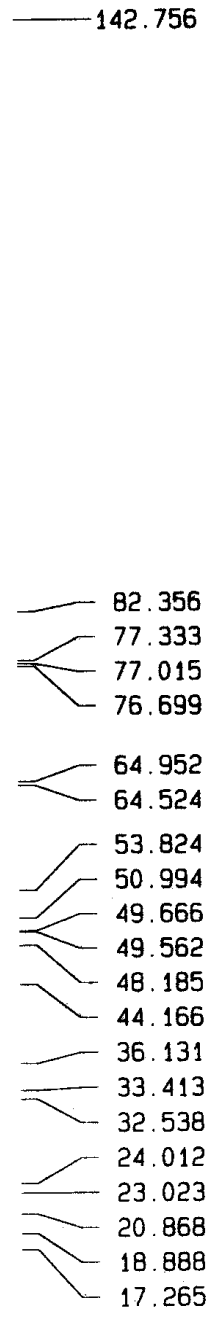
Figure S46. Expanded ${ }^{13} \mathrm{C}$ NMR spectrum of daphniyunnine E (5) in $\mathrm{CDCl}_{3}\left(\delta_{\mathrm{C}} 47-67\right)$
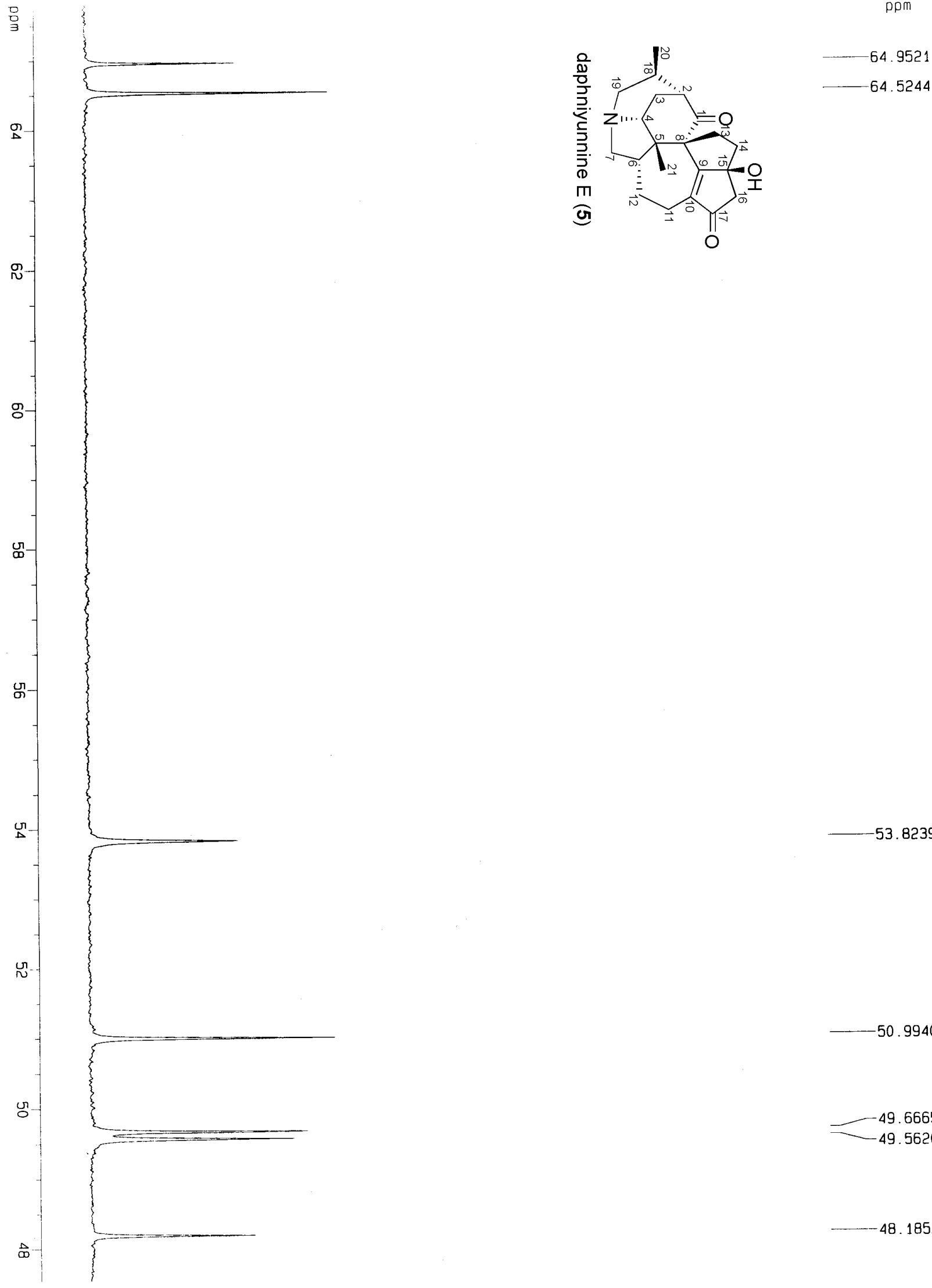
Figure S47. DEPT spectrum of daphniyunnine E (5) in $\mathrm{CDCl}_{3}$

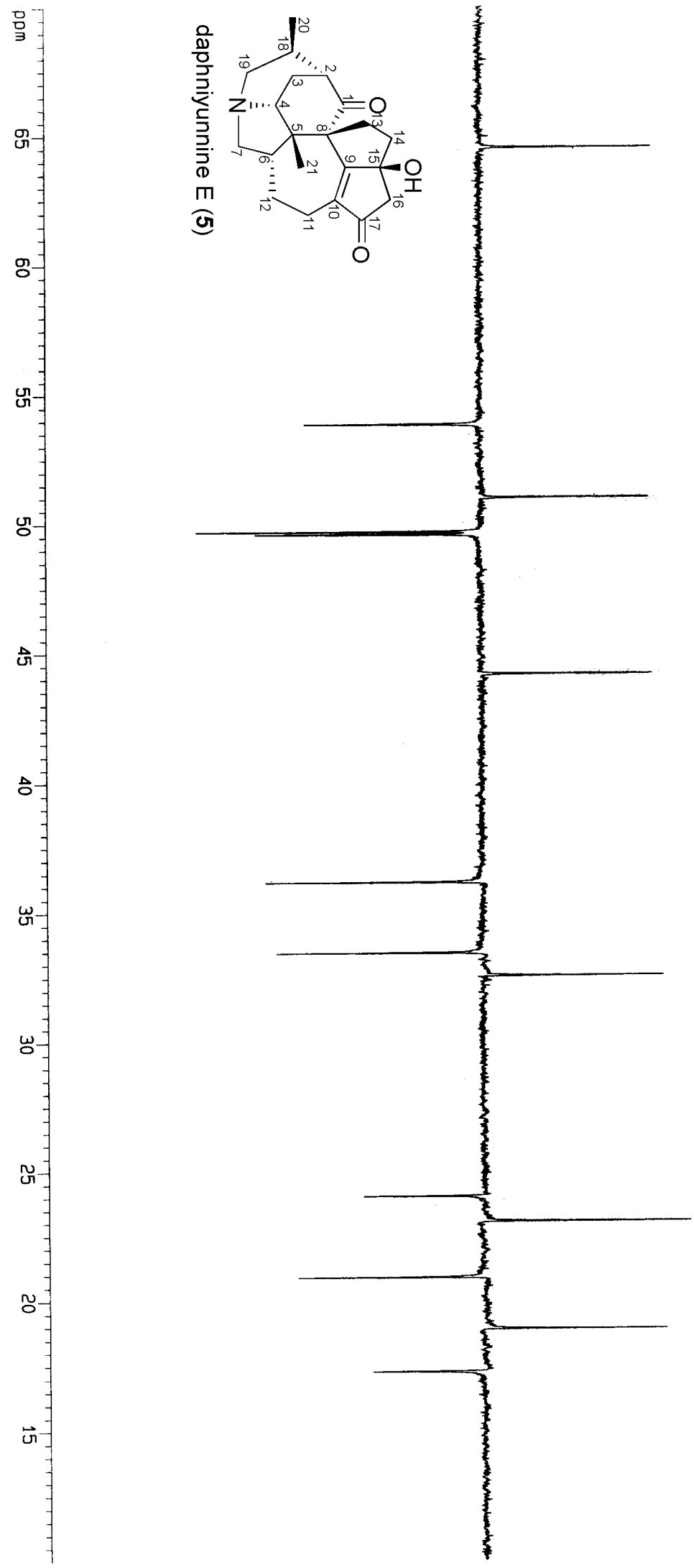

ppm

64.5197

53.8224

50.9928

49.6654

$-44.1605$

$-36.1354$

- 33.4129

32.5370

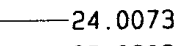

23.0309

$-20.8682$

$-18.8920$

$-17.2594$ 
Figure S48. EIMS spectrum of daphniyunnine E (5)

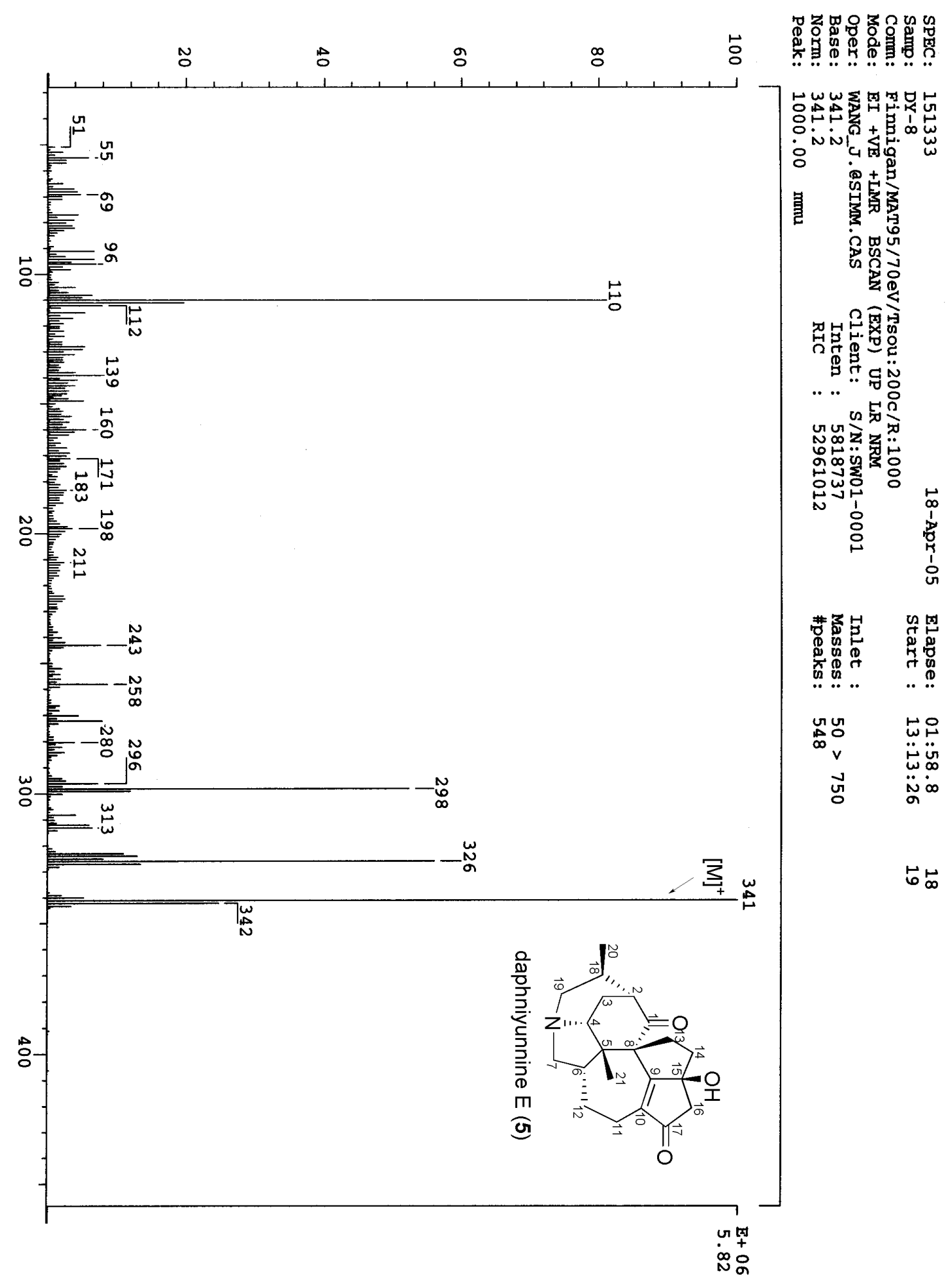


Figure S49. IR spectrum of daphniyunnine E (5)

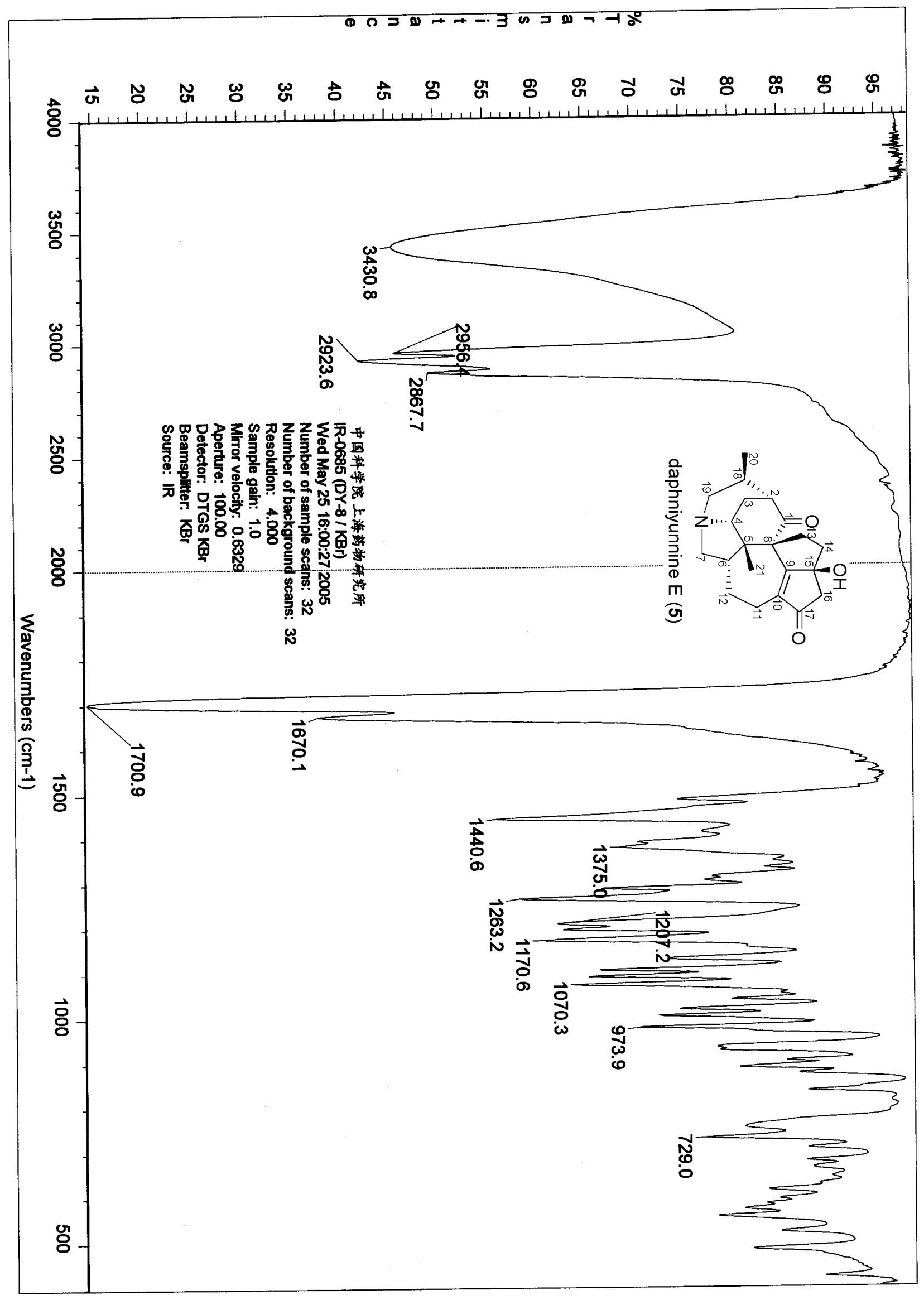


Figure S50. ${ }^{1} \mathrm{H}-{ }^{1} \mathrm{H}$ COSY spectrum of daphniyunnine $\mathrm{E}$ (5) in $\mathrm{CDCl}_{3}$

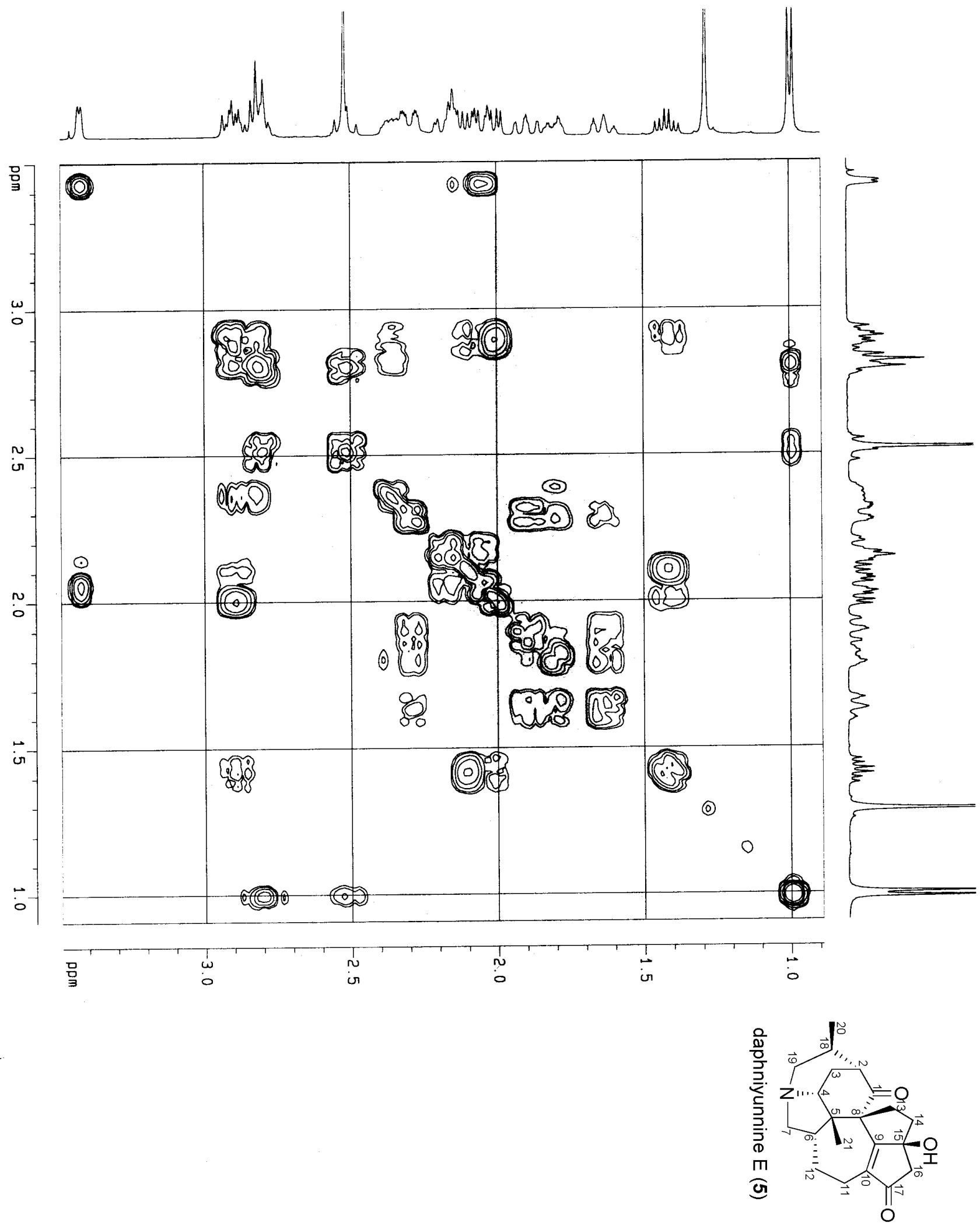


Figure S51. HMQC spectrum of daphniyunnine E (5) in $\mathrm{CDCl}_{3}$

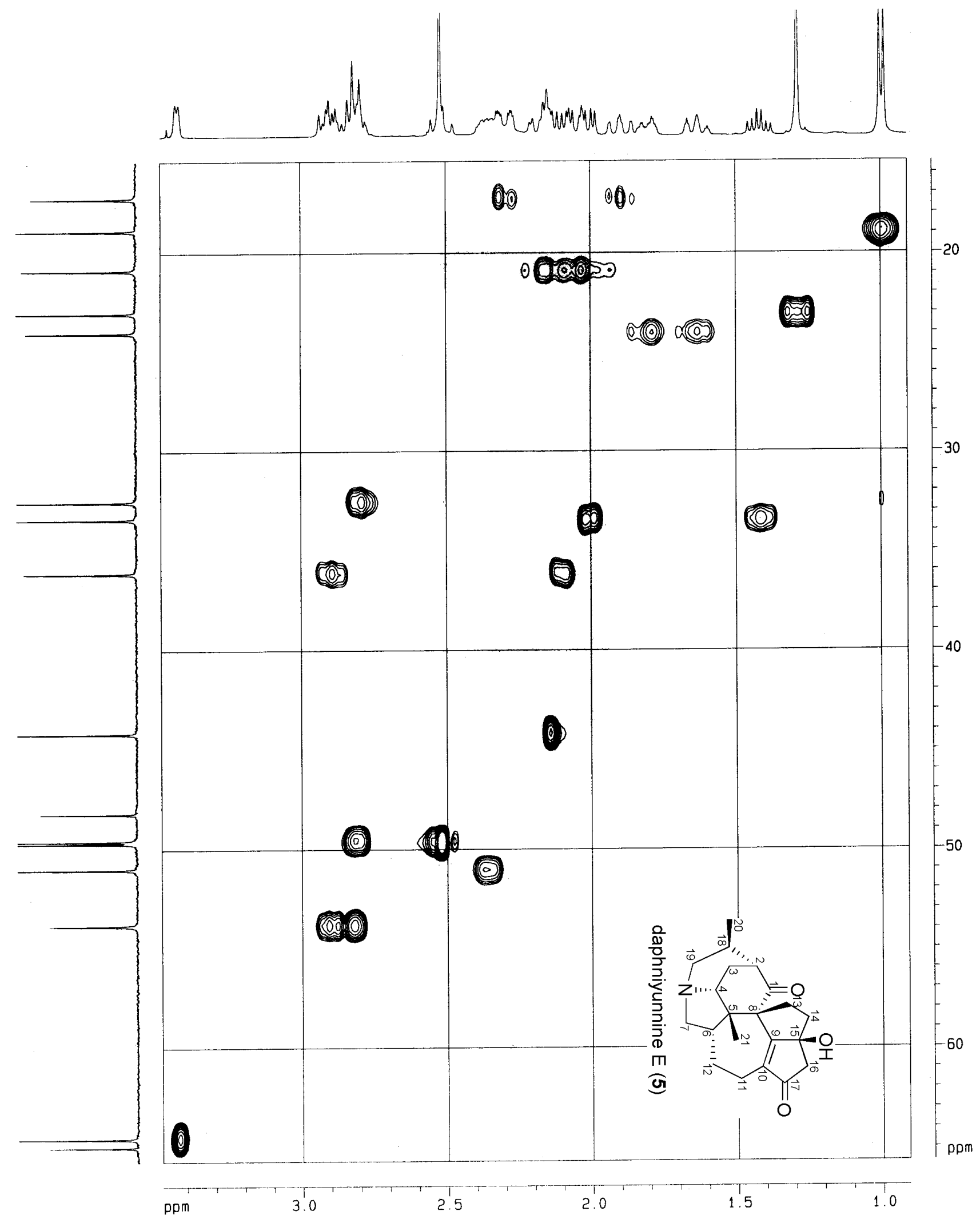


Figure S52. HMBC spectrum of daphniyunnine E (5) in $\mathrm{CDCl}_{3}$

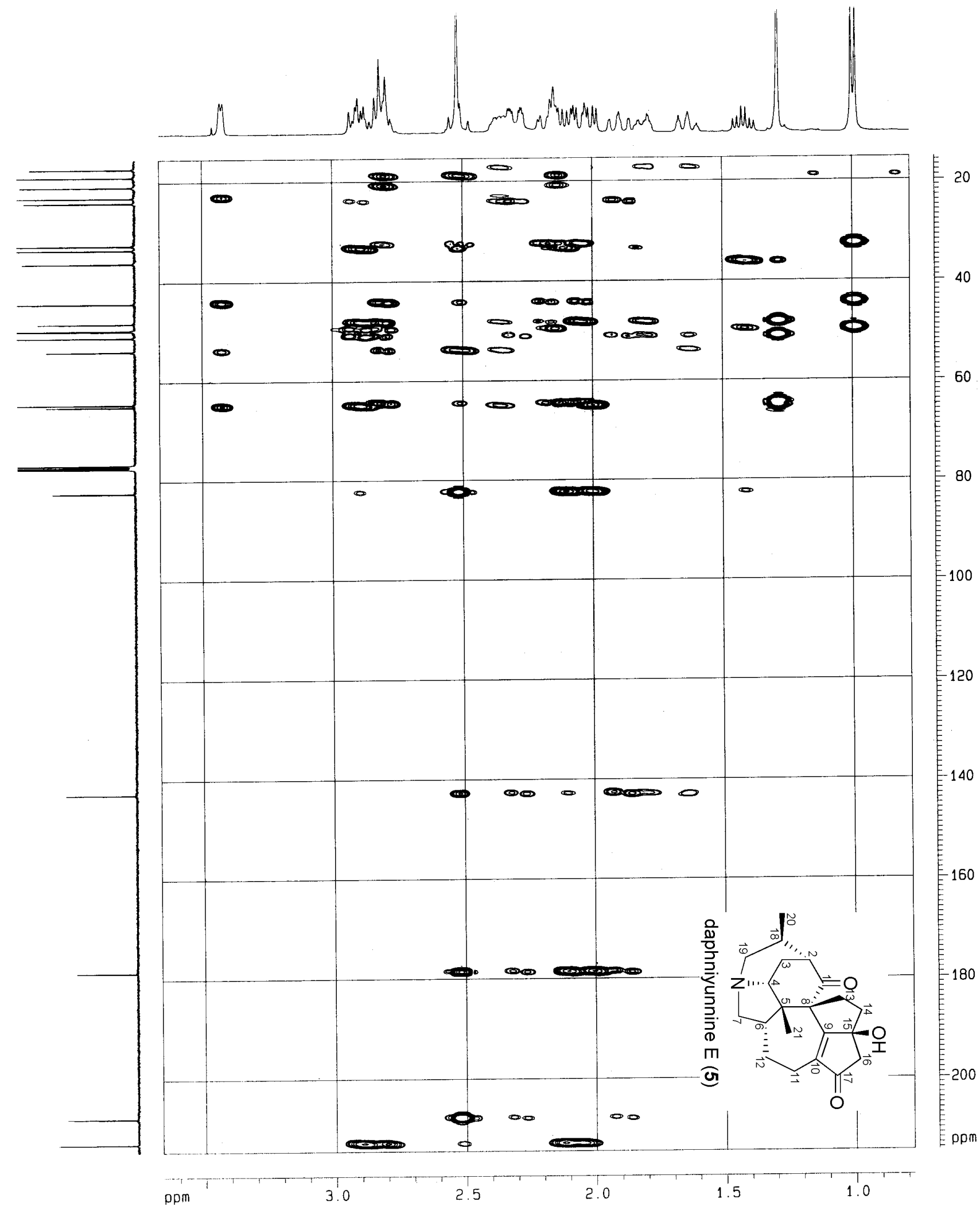


Figure S53. NOESY spectrum of daphniyunnine E (5) in $\mathrm{CDCl}_{3}$

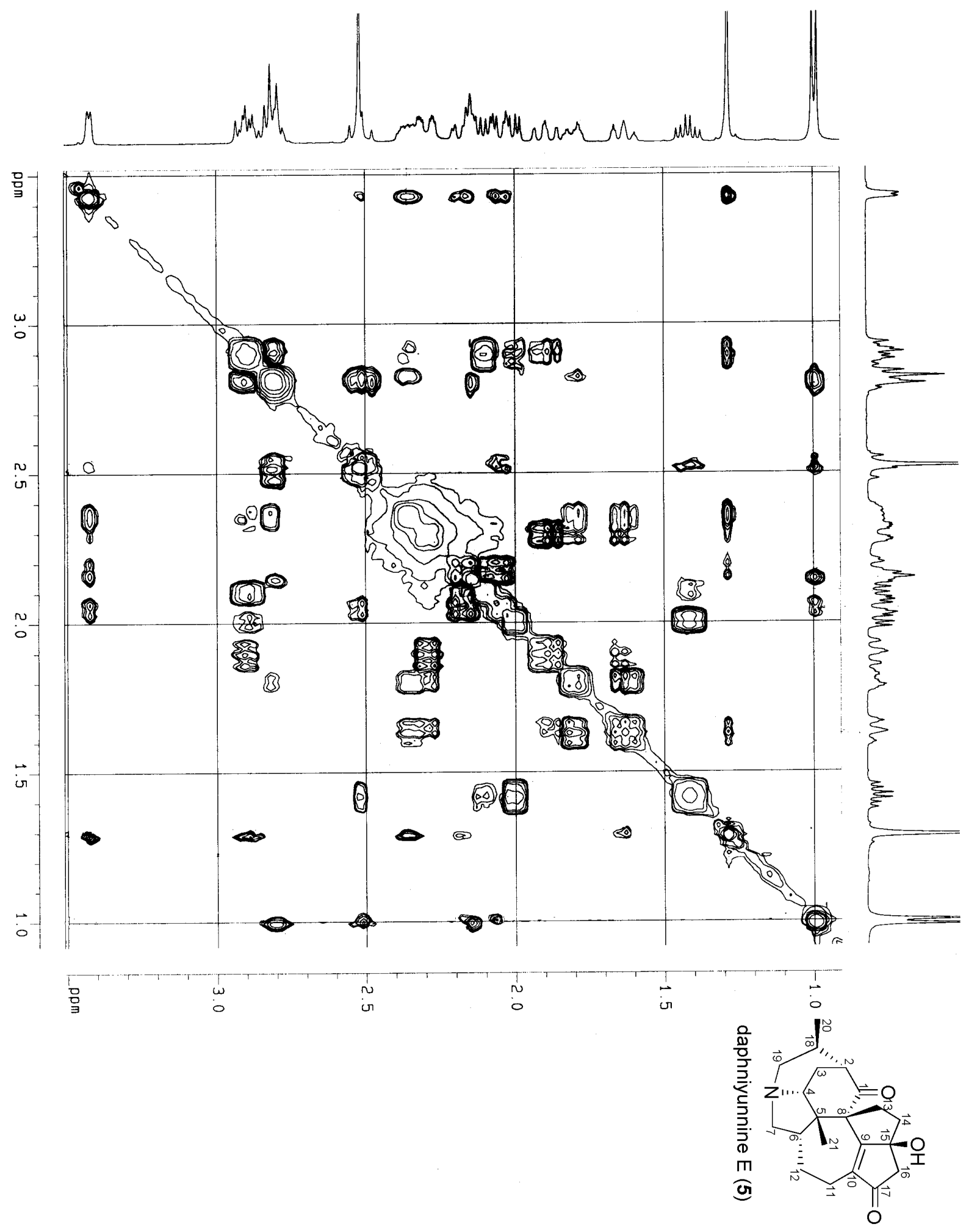




\section{X-ray data tables for daphniyunnine A (1):}

Table 1. Crystal data and structure refinement for cd25123.

Identification code

Empirical formula

Formula weight

Temperature

Wavelength

Crystal system, space group

Unit cell dimensions

Volume

Z, Calculated density

Absorption coefficient

$\mathrm{F}(000)$

Crystal size

Theta range for data collection

Limiting indices

Reflections collected / unique

Completeness to theta $=26.99$

Absorption correction

Max. and min. transmission

Refinement method

Data / restraints / parameters

Goodness-of-fit on $\mathrm{F}^{\wedge} 2$

Final $R$ indices $[\mathrm{I}>2$ sigma (I)

$\mathrm{R}$ indices (all data)

Absolute structure parameter

Extinction coefficient

Largest diff. peak and hole $\operatorname{cd} 25123$

C23 H31 N 03

369. 49

$293(2) \quad \mathrm{K}$

$0.71073 \mathrm{~A}$

Orthorhombic, P2(1)2(1)2(1)

$\mathrm{a}=6.8007(7)$ A alpha $=90 \mathrm{deg}$.

$\mathrm{b}=11.2573(11) \mathrm{A} \quad$ beta $=90 \mathrm{deg}$.

$\mathrm{c}=24.747(2) \mathrm{A} \quad$ gamma $=90 \mathrm{deg}$.

1894. $6(3)$ A`3

4, $1.295 \mathrm{Mg} / \mathrm{m}^{\wedge} 3$

$0.085 \mathrm{~mm}^{\wedge}-1$

800

$0.517 \times 0.304 \times 0.289 \mathrm{~mm}$

1.65 to $26.99 \mathrm{deg}$.

$-8<=\mathrm{h}<=8, \quad-14<=\mathrm{k}<=9, \quad-31<=1<=31$

$11203 / 4129[\mathrm{R}($ int $)=0.1329]$

$100.0 \%$

Empirical

1.00000 and 0.79019

Ful1-matrix least-squares on $\mathrm{F}^{\wedge} 2$

$4129 / 0$ / 369

0.922

$\mathrm{R} 1=0.0502, \quad w R 2=0.1012$

$\mathrm{R} 1=0.0640, \quad \mathrm{wR} 2=0.1066$

$-0.3(14)$

$0.0099(11)$

0.242 and -0.220 e. $A^{\wedge}-3$ 
Table 2. Atomic coordinates ( x 10^4) and equivalent isotropic displacement parameters (A^2 x 10^3) for cd25123.

$\mathrm{U}(\mathrm{eq})$ is defined as one third of the trace of the orthogonalized Uij tensor.

\begin{tabular}{|c|c|c|c|c|}
\hline & $\mathrm{x}$ & $\mathrm{y}$ & z & $\mathrm{U}(\mathrm{eq})$ \\
\hline $0(1)$ & $4892(2)$ & $3663(2)$ & $7958(1)$ & $44(1)$ \\
\hline $0(2)$ & $-650(3)$ & $5602(2)$ & $9431(1)$ & $62(1)$ \\
\hline $0(3)$ & $1815(3)$ & $6879(2)$ & $9347(1)$ & $55(1)$ \\
\hline $\mathrm{N}(1)$ & $1917(3)$ & $1138(2)$ & $7497(1)$ & $42(1)$ \\
\hline $\mathrm{C}(1)$ & $3167(3)$ & $3435(2)$ & $7904(1)$ & $29(1)$ \\
\hline $\mathrm{C}(2)$ & $2251(3)$ & $3485(2)$ & $7343(1)$ & $35(1)$ \\
\hline $\mathrm{C}(3)$ & $163(3)$ & $3012(2)$ & $7347(1)$ & $38(1)$ \\
\hline $\mathrm{C}(4)$ & $232(3)$ & $1853(2)$ & $7665(1)$ & $34(1)$ \\
\hline $\mathrm{C}(5)$ & $522(3)$ & $2030(2)$ & $8284(1)$ & $31(1)$ \\
\hline$C(6)$ & $1533(3)$ & $832(2)$ & $8405(1)$ & $31(1)$ \\
\hline $\mathrm{C}(7)$ & 3051 (4) & $721(2)$ & $7963(1)$ & $35(1)$ \\
\hline $\mathrm{C}(8)$ & $1847(3)$ & $3154(2)$ & $8393(1)$ & $27(1)$ \\
\hline C (9) & $3090(3)$ & $3164(2)$ & $8896(1)$ & $27(1)$ \\
\hline$C(10)$ & $3800(3)$ & $2397(2)$ & $9254(1)$ & $32(1)$ \\
\hline $\mathrm{C}(11)$ & $3837(4)$ & $1079(2)$ & $9246(1)$ & $39(1)$ \\
\hline$C(12)$ & $2083(4)$ & $492(2)$ & $8982(1)$ & $39(1)$ \\
\hline $\mathrm{C}(13)$ & $637(4)$ & $4329(2)$ & $8484(1)$ & $41(1)$ \\
\hline $\mathrm{C}(14)$ & $1963(4)$ & $5205(2)$ & $8795(1)$ & $37(1)$ \\
\hline$C(15)$ & $3600(4)$ & $4422(2)$ & $9050(1)$ & $35(1)$ \\
\hline$C(16)$ & $3960(4)$ & $4325(2)$ & $9657(1)$ & $47(1)$ \\
\hline$C(17)$ & $4832(4)$ & 3087 (2) & $9703(1)$ & $41(1)$ \\
\hline $\mathrm{C}(18)$ & $3533(4)$ & $2749(2)$ & $6955(1)$ & $41(1)$ \\
\hline $\mathrm{C}(19)$ & $2911(5)$ & $1452(2)$ & $6998(1)$ & $47(1)$ \\
\hline$C(20)$ & $3393(6)$ & $3166(3)$ & $6371(1)$ & $64(1)$ \\
\hline $\mathrm{C}(21)$ & $-1450(3)$ & $2087(3)$ & $8573(1)$ & $47(1)$ \\
\hline $\mathrm{C}(22)$ & $847(4)$ & $5889(2)$ & $9220(1)$ & $41(1)$ \\
\hline $\mathrm{C}(23)$ & $952(6)$ & $7600(3)$ & $9768(1)$ & $63(1)$ \\
\hline
\end{tabular}


Table 3. Bond lengths [A] and angles [deg] for cd25123.

\begin{tabular}{|c|c|}
\hline $0(1)-C(1)$ & 1. $208(3)$ \\
\hline $0(2)-C(22)$ & 1. $188(3)$ \\
\hline $0(3)-C(22)$ & $1.331(3)$ \\
\hline $0(3)-C(23)$ & 1. $445(3)$ \\
\hline$N(1)-C(19)$ & 1. $452(3)$ \\
\hline $\mathrm{N}(1)-\mathrm{C}(4)$ & 1. $461(3)$ \\
\hline $\mathrm{N}(1)-\mathrm{C}(7)$ & 1. $466(3)$ \\
\hline $\mathrm{C}(1)-\mathrm{C}(2)$ & $1.522(3)$ \\
\hline$C(1)-C(8)$ & 1. $540(3)$ \\
\hline$C(2)-C(3)$ & $1.517(3)$ \\
\hline$C(2)-C(18)$ & 1. $539(3)$ \\
\hline $\mathrm{C}(2)-\mathrm{H}(1)$ & $1.00(2)$ \\
\hline$C(3)-C(4)$ & 1. $526(3)$ \\
\hline $\mathrm{C}(3)-\mathrm{H}(2)$ & $0.96(2)$ \\
\hline $\mathrm{C}(3)-\mathrm{H}(3)$ & $1.05(3)$ \\
\hline$C(4)-C(5)$ & $1.558(3)$ \\
\hline $\mathrm{C}(4)-\mathrm{H}(4)$ & 1. $124(16)$ \\
\hline$C(5)-C(21)$ & 1. $521(3)$ \\
\hline$C(5)-C(6)$ & $1.544(3)$ \\
\hline$C(5)-C(8)$ & $1.576(3)$ \\
\hline$C(6)-C(7)$ & $1.509(3)$ \\
\hline$C(6)-C(12)$ & 1. $525(3)$ \\
\hline $\mathrm{C}(6)-\mathrm{H}(5)$ & $1.00(2)$ \\
\hline $\mathrm{C}(7)-\mathrm{H}(6)$ & 1. $04(2)$ \\
\hline $\mathrm{C}(7)-\mathrm{H}(7)$ & $1.03(2)$ \\
\hline$C(8)-C(9)$ & 1. $504(3)$ \\
\hline$C(8)-C(13)$ & $1.574(3)$ \\
\hline$C(9)-C(10)$ & 1. $327(3)$ \\
\hline$C(9)-C(15)$ & $1.507(3)$ \\
\hline$C(10)-C(11)$ & 1. $484(3)$ \\
\hline$C(10)-C(17)$ & 1. $527(3)$ \\
\hline$C(11)-C(12)$ & $1.511(3)$ \\
\hline $\mathrm{C}(11)-\mathrm{H}(8)$ & $0.96(3)$ \\
\hline $\mathrm{C}(11)-\mathrm{H}(9)$ & $0.99(2)$ \\
\hline $\mathrm{C}(12)-\mathrm{H}(10)$ & $0.97(2)$ \\
\hline $\mathrm{C}(12)-\mathrm{H}(11)$ & $1.00(2)$ \\
\hline$C(13)-C(14)$ & 1. $541(3)$ \\
\hline $\mathrm{C}(13)-\mathrm{H}(12)$ & $1.01(3)$ \\
\hline $\mathrm{C}(13)-\mathrm{H}(13)$ & $0.98(3)$ \\
\hline$C(14)-C(22)$ & $1.509(3)$ \\
\hline$C(14)-C(15)$ & 1. $554(3)$ \\
\hline $\mathrm{C}(14)-\mathrm{H}(14)$ & $0.99(3)$ \\
\hline$C(15)-C(16)$ & 1. $527(3)$ \\
\hline $\mathrm{C}(15)-\mathrm{H}(15)$ & $0.95(2)$ \\
\hline
\end{tabular}




\begin{tabular}{|c|c|}
\hline$C(16)-C(17)$ & $1.519(4)$ \\
\hline $\mathrm{C}(16)-\mathrm{H}(16)$ & $0.96(3)$ \\
\hline $\mathrm{C}(16)-\mathrm{H}(17)$ & $1.00(2)$ \\
\hline $\mathrm{C}(17)-\mathrm{H}(18)$ & $0.965(19)$ \\
\hline $\mathrm{C}(17)-\mathrm{H}(19)$ & $0.95(3)$ \\
\hline$C(18)-C(20)$ & 1. $522(3)$ \\
\hline$C(18)-C(19)$ & 1. $524(3)$ \\
\hline $\mathrm{C}(18)-\mathrm{H}(20)$ & $0.99(2)$ \\
\hline $\mathrm{C}(19)-\mathrm{H}(21)$ & $0.91(3)$ \\
\hline $\mathrm{C}(19)-\mathrm{H}(22)$ & $1.05(3)$ \\
\hline $\mathrm{C}(20)-\mathrm{H}(23)$ & $0.98(3)$ \\
\hline $\mathrm{C}(20)-\mathrm{H}(24)$ & $1.00(4)$ \\
\hline $\mathrm{C}(20)-\mathrm{H}(25)$ & $1.00(4)$ \\
\hline $\mathrm{C}(21)-\mathrm{H}(26)$ & $1.01(2)$ \\
\hline $\mathrm{C}(21)-\mathrm{H}(27)$ & $0.99(3)$ \\
\hline $\mathrm{C}(21)-\mathrm{H}(28)$ & 1. $05(3)$ \\
\hline $\mathrm{C}(23)-\mathrm{H}(29)$ & $0.94(3)$ \\
\hline $\mathrm{C}(23)-\mathrm{H}(30)$ & $1.04(3)$ \\
\hline $\mathrm{C}(23)-\mathrm{H}(31)$ & $0.91(4)$ \\
\hline$C(22)-0(3)-C(23)$ & $116.1(2)$ \\
\hline $\mathrm{C}(19)-\mathrm{N}(1)-\mathrm{C}(4)$ & 118. $28(19)$ \\
\hline $\mathrm{C}(19)-\mathrm{N}(1)-\mathrm{C}(7)$ & $120.2(2)$ \\
\hline $\mathrm{C}(4)-\mathrm{N}(1)-\mathrm{C}(7)$ & $111.32(16)$ \\
\hline $0(1)-C(1)-C(2)$ & 119. $43(19)$ \\
\hline $0(1)-C(1)-C(8)$ & $121.41(18)$ \\
\hline$C(2)-C(1)-C(8)$ & 119. $09(18)$ \\
\hline$C(3)-C(2)-C(1)$ & $111.41(18)$ \\
\hline$C(3)-C(2)-C(18)$ & $110.17(18)$ \\
\hline$C(1)-C(2)-C(18)$ & $108.50(18)$ \\
\hline $\mathrm{C}(3)-\mathrm{C}(2)-\mathrm{H}(1)$ & 113. $2(11)$ \\
\hline $\mathrm{C}(1)-\mathrm{C}(2)-\mathrm{H}(1)$ & $104.3(11)$ \\
\hline $\mathrm{C}(18)-\mathrm{C}(2)-\mathrm{H}(1)$ & 109. $0(11)$ \\
\hline$C(2)-C(3)-C(4)$ & $105.91(17)$ \\
\hline $\mathrm{C}(2)-\mathrm{C}(3)-\mathrm{H}(2)$ & 108. $6(13)$ \\
\hline $\mathrm{C}(4)-\mathrm{C}(3)-\mathrm{H}(2)$ & $109.1(14)$ \\
\hline $\mathrm{C}(2)-\mathrm{C}(3)-\mathrm{H}(3)$ & $116.7(13)$ \\
\hline $\mathrm{C}(4)-\mathrm{C}(3)-\mathrm{H}(3)$ & 108. $3(14)$ \\
\hline $\mathrm{H}(2)-\mathrm{C}(3)-\mathrm{H}(3)$ & 108. $0(19)$ \\
\hline $\mathrm{N}(1)-\mathrm{C}(4)-\mathrm{C}(3)$ & $110.34(18)$ \\
\hline$N(1)-C(4)-C(5)$ & 104. $62(16)$ \\
\hline$C(3)-C(4)-C(5)$ & $113.75(18)$ \\
\hline $\mathrm{N}(1)-\mathrm{C}(4)-\mathrm{H}(4)$ & $115.6(8)$ \\
\hline $\mathrm{C}(3)-\mathrm{C}(4)-\mathrm{H}(4)$ & 106. $2(8)$ \\
\hline $\mathrm{C}(5)-\mathrm{C}(4)-\mathrm{H}(4)$ & $106.5(8)$ \\
\hline$C(21)-C(5)-C(6)$ & 109. $80(19)$ \\
\hline$C(21)-C(5)-C(4)$ & $110.78(17)$ \\
\hline$C(6)-C(5)-C(4)$ & $97.75(16)$ \\
\hline$C(21)-C(5)-C(8)$ & $112.99(19)$ \\
\hline
\end{tabular}




\begin{tabular}{|c|c|}
\hline$C(6)-C(5)-C(8)$ & 114. $45(15)$ \\
\hline$C(4)-C(5)-C(8)$ & 110. $08(16)$ \\
\hline$C(7)-C(6)-C(12)$ & 119. $4(2)$ \\
\hline$C(7)-C(6)-C(5)$ & 103. $65(16)$ \\
\hline$C(12)-C(6)-C(5)$ & 120. $65(18)$ \\
\hline $\mathrm{C}(7)-\mathrm{C}(6)-\mathrm{H}(5)$ & 109. $4(12)$ \\
\hline $\mathrm{C}(12)-\mathrm{C}(6)-\mathrm{H}(5)$ & $102.9(13)$ \\
\hline $\mathrm{C}(5)-\mathrm{C}(6)-\mathrm{H}(5)$ & $98.5(12)$ \\
\hline$N(1)-C(7)-C(6)$ & $100.68(18)$ \\
\hline$N(1)-C(7)-H(6)$ & $112.0(14)$ \\
\hline $\mathrm{C}(6)-\mathrm{C}(7)-\mathrm{H}(6)$ & $117.6(13)$ \\
\hline$N(1)-C(7)-H(7)$ & 113. $2(12)$ \\
\hline $\mathrm{C}(6)-\mathrm{C}(7)-\mathrm{H}(7)$ & $114.7(11)$ \\
\hline $\mathrm{H}(6)-\mathrm{C}(7)-\mathrm{H}(7)$ & $99.3(17)$ \\
\hline$C(9)-C(8)-C(1)$ & 108. $78(15)$ \\
\hline C (9) $-C(8)-C(13)$ & $99.77(15)$ \\
\hline$C(1)-C(8)-C(13)$ & $104.17(16)$ \\
\hline $\mathrm{C}(9)-\mathrm{C}(8)-\mathrm{C}(5)$ & $117.90(16)$ \\
\hline$C(1)-C(8)-C(5)$ & $111.30(15)$ \\
\hline$C(13)-C(8)-C(5)$ & 113. $57(16)$ \\
\hline$C(10)-C(9)-C(8)$ & 138. $71(19)$ \\
\hline$C(10)-C(9)-C(15)$ & $111.09(17)$ \\
\hline$C(8)-C(9)-C(15)$ & $110.14(16)$ \\
\hline$C(9)-C(10)-C(11)$ & $130.4(2)$ \\
\hline$C(9)-C(10)-C(17)$ & $108.8(2)$ \\
\hline$C(11)-C(10)-C(17)$ & $120.7(2)$ \\
\hline$C(10)-C(11)-C(12)$ & $115.4(2)$ \\
\hline $\mathrm{C}(10)-\mathrm{C}(11)-\mathrm{H}(8)$ & 109. $0(15)$ \\
\hline $\mathrm{C}(12)-\mathrm{C}(11)-\mathrm{H}(8)$ & 106. 4(15) \\
\hline $\mathrm{C}(10)-\mathrm{C}(11)-\mathrm{H}(9)$ & $103.9(14)$ \\
\hline $\mathrm{C}(12)-\mathrm{C}(11)-\mathrm{H}(9)$ & $114.3(14)$ \\
\hline $\mathrm{H}(8)-\mathrm{C}(11)-\mathrm{H}(9)$ & $108(2)$ \\
\hline$C(11)-C(12)-C(6)$ & $119.2(2)$ \\
\hline $\mathrm{C}(11)-\mathrm{C}(12)-\mathrm{H}(10)$ & $110.0(13)$ \\
\hline $\mathrm{C}(6)-\mathrm{C}(12)-\mathrm{H}(10)$ & $107.3(14)$ \\
\hline $\mathrm{C}(11)-\mathrm{C}(12)-\mathrm{H}(11)$ & 106. $0(13)$ \\
\hline $\mathrm{C}(6)-\mathrm{C}(12)-\mathrm{H}(11)$ & 107. $7(14)$ \\
\hline $\mathrm{H}(10)-\mathrm{C}(12)-\mathrm{H}(11)$ & 105. 9(19) \\
\hline$C(14)-C(13)-C(8)$ & $107.63(18)$ \\
\hline $\mathrm{C}(14)-\mathrm{C}(13)-\mathrm{H}(12)$ & $112.1(16)$ \\
\hline $\mathrm{C}(8)-\mathrm{C}(13)-\mathrm{H}(12)$ & $112.3(16)$ \\
\hline $\mathrm{C}(14)-\mathrm{C}(13)-\mathrm{H}(13)$ & 107. 4(16) \\
\hline $\mathrm{C}(8)-\mathrm{C}(13)-\mathrm{H}(13)$ & $110.2(16)$ \\
\hline $\mathrm{H}(12)-\mathrm{C}(13)-\mathrm{H}(13)$ & $107(2)$ \\
\hline$C(22)-C(14)-C(13)$ & $112.4(2)$ \\
\hline$C(22)-C(14)-C(15)$ & $111.52(18)$ \\
\hline$C(13)-C(14)-C(15)$ & 105. $01(18)$ \\
\hline $\mathrm{C}(22)-\mathrm{C}(14)-\mathrm{H}(14)$ & $102.0(15)$ \\
\hline $\mathrm{C}(13)-\mathrm{C}(14)-\mathrm{H}(14)$ & 115. 1 (15) \\
\hline
\end{tabular}




\begin{tabular}{|c|c|}
\hline $\mathrm{C}(15)-\mathrm{C}(14)-\mathrm{H}(14)$ & $111.1(14)$ \\
\hline$C(9)-C(15)-C(16)$ & $102.55(18)$ \\
\hline$C(9)-C(15)-C(14)$ & 105. $41(17)$ \\
\hline$C(16)-C(15)-C(14)$ & $123.73(19)$ \\
\hline $\mathrm{C}(9)-\mathrm{C}(15)-\mathrm{H}(15)$ & $113.7(14)$ \\
\hline $\mathrm{C}(16)-\mathrm{C}(15)-\mathrm{H}(15)$ & 107. 2(14) \\
\hline $\mathrm{C}(14)-\mathrm{C}(15)-\mathrm{H}(15)$ & $104.5(14)$ \\
\hline$C(17)-C(16)-C(15)$ & $101.69(19)$ \\
\hline $\mathrm{C}(17)-\mathrm{C}(16)-\mathrm{H}(16)$ & 114. $3(15)$ \\
\hline $\mathrm{C}(15)-\mathrm{C}(16)-\mathrm{H}(16)$ & $111.8(16)$ \\
\hline $\mathrm{C}(17)-\mathrm{C}(16)-\mathrm{H}(17)$ & 105. 1(16) \\
\hline $\mathrm{C}(15)-\mathrm{C}(16)-\mathrm{H}(17)$ & $111.4(15)$ \\
\hline $\mathrm{H}(16)-\mathrm{C}(16)-\mathrm{H}(17)$ & $112(2)$ \\
\hline$C(16)-C(17)-C(10)$ & $103.43(18)$ \\
\hline $\mathrm{C}(16)-\mathrm{C}(17)-\mathrm{H}(18)$ & 109. 1(13) \\
\hline $\mathrm{C}(10)-\mathrm{C}(17)-\mathrm{H}(18)$ & 113. $2(12)$ \\
\hline$C(16)-C(17)-H(19)$ & $111.5(16)$ \\
\hline $\mathrm{C}(10)-\mathrm{C}(17)-\mathrm{H}(19)$ & $111.7(16)$ \\
\hline $\mathrm{H}(18)-\mathrm{C}(17)-\mathrm{H}(19)$ & 107. 8(18) \\
\hline$C(20)-C(18)-C(19)$ & $110.1(2)$ \\
\hline$C(20)-C(18)-C(2)$ & $113.1(2)$ \\
\hline$C(19)-C(18)-C(2)$ & 108. $38(19)$ \\
\hline$C(20)-C(18)-H(20)$ & $107.1(12)$ \\
\hline $\mathrm{C}(19)-\mathrm{C}(18)-\mathrm{H}(20)$ & 106. 6(12) \\
\hline $\mathrm{C}(2)-\mathrm{C}(18)-\mathrm{H}(20)$ & $111.4(12)$ \\
\hline $\mathrm{N}(1)-\mathrm{C}(19)-\mathrm{C}(18)$ & $114.94(18)$ \\
\hline $\mathrm{N}(1)-\mathrm{C}(19)-\mathrm{H}(21)$ & $108.0(18)$ \\
\hline $\mathrm{C}(18)-\mathrm{C}(19)-\mathrm{H}(21)$ & 106. $3(18)$ \\
\hline $\mathrm{N}(1)-\mathrm{C}(19)-\mathrm{H}(22)$ & 108. 4(16) \\
\hline $\mathrm{C}(18)-\mathrm{C}(19)-\mathrm{H}(22)$ & $108.0(15)$ \\
\hline $\mathrm{H}(21)-\mathrm{C}(19)-\mathrm{H}(22)$ & $111(2)$ \\
\hline $\mathrm{C}(18)-\mathrm{C}(20)-\mathrm{H}(23)$ & $107.7(17)$ \\
\hline $\mathrm{C}(18)-\mathrm{C}(20)-\mathrm{H}(24)$ & $108(2)$ \\
\hline $\mathrm{H}(23)-\mathrm{C}(20)-\mathrm{H}(24)$ & $120(3)$ \\
\hline $\mathrm{C}(18)-\mathrm{C}(20)-\mathrm{H}(25)$ & 105. $0(18)$ \\
\hline $\mathrm{H}(23)-\mathrm{C}(20)-\mathrm{H}(25)$ & $112(3)$ \\
\hline $\mathrm{H}(24)-\mathrm{C}(20)-\mathrm{H}(25)$ & $104(3)$ \\
\hline $\mathrm{C}(5)-\mathrm{C}(21)-\mathrm{H}(26)$ & 113. 8(13) \\
\hline $\mathrm{C}(5)-\mathrm{C}(21)-\mathrm{H}(27)$ & 107. 8(12) \\
\hline $\mathrm{H}(26)-\mathrm{C}(21)-\mathrm{H}(27)$ & 107.2(18) \\
\hline $\mathrm{C}(5)-\mathrm{C}(21)-\mathrm{H}(28)$ & $112.4(14)$ \\
\hline $\mathrm{H}(26)-\mathrm{C}(21)-\mathrm{H}(28)$ & 104. 2(17) \\
\hline $\mathrm{H}(27)-\mathrm{C}(21)-\mathrm{H}(28)$ & 111. 3(19) \\
\hline $0(2)-C(22)-0(3)$ & $123.2(2)$ \\
\hline$O(2)-C(22)-C(14)$ & $126.7(2)$ \\
\hline $0(3)-C(22)-C(14)$ & $110.1(2)$ \\
\hline $0(3)-\mathrm{C}(23)-\mathrm{H}(29)$ & $113.6(18)$ \\
\hline $0(3)-\mathrm{C}(23)-\mathrm{H}(30)$ & 106. 4(16) \\
\hline $\mathrm{H}(29)-\mathrm{C}(23)-\mathrm{H}(30)$ & $109(2)$ \\
\hline
\end{tabular}




$\begin{array}{ll}0(3)-\mathrm{C}(23)-\mathrm{H}(31) & 111(2) \\ \mathrm{H}(29)-\mathrm{C}(23)-\mathrm{H}(31) & 111(3) \\ \mathrm{H}(30)-\mathrm{C}(23)-\mathrm{H}(31) & 106(3)\end{array}$

Symmetry transformations used to generate equivalent atoms:

Table 4. Anisotropic displacement parameters (A`2 x 10^3) for cd25123. The anisotropic displacement factor exponent takes the form:

-2 pi`$^{\wedge}\left[\mathrm{h}^{\wedge} 2 \mathrm{a}^{\wedge} 2 \mathrm{U} 11+\ldots+2 \mathrm{~h} \mathrm{k}\right.$ a* b* U12 ]

\begin{tabular}{|c|c|c|c|c|c|c|}
\hline & U11 & U22 & U33 & U23 & U13 & U12 \\
\hline $0(1)$ & $46(1)$ & $58(1)$ & $27(1)$ & $2(1)$ & $-2(1)$ & $-16(1)$ \\
\hline $0(2)$ & $70(1)$ & $60(1)$ & $55(1)$ & $-17(1)$ & $19(1)$ & $-3(1)$ \\
\hline $0(3)$ & $77(1)$ & $39(1)$ & $50(1)$ & $-21(1)$ & $5(1)$ & $3(1)$ \\
\hline $\mathrm{N}(1)$ & $66(1)$ & $37(1)$ & $22(1)$ & $1(1)$ & $7(1)$ & $6(1)$ \\
\hline$C(1)$ & $43(1)$ & $20(1)$ & $24(1)$ & $-1(1)$ & $-1(1)$ & $0(1)$ \\
\hline$C(2)$ & $58(1)$ & $25(1)$ & $21(1)$ & $2(1)$ & $-3(1)$ & $-3(1)$ \\
\hline C (3) & $53(1)$ & $42(1)$ & $20(1)$ & $0(1)$ & $-14(1)$ & $5(1)$ \\
\hline $\mathrm{C}(4)$ & $40(1)$ & $36(1)$ & $26(1)$ & $-2(1)$ & $-6(1)$ & $-5(1)$ \\
\hline$C(5)$ & $32(1)$ & $36(1)$ & $24(1)$ & $1(1)$ & $0(1)$ & $-1(1)$ \\
\hline$C(6)$ & $38(1)$ & $30(1)$ & $26(1)$ & $1(1)$ & $1(1)$ & $-6(1)$ \\
\hline$C(7)$ & $49(1)$ & $28(1)$ & $27(1)$ & $-1(1)$ & $4(1)$ & $3(1)$ \\
\hline C (8) & $34(1)$ & $28(1)$ & $20(1)$ & $-2(1)$ & $-2(1)$ & $6(1)$ \\
\hline C (9) & $33(1)$ & $31(1)$ & $17(1)$ & $-2(1)$ & $3(1)$ & $1(1)$ \\
\hline$C(10)$ & $36(1)$ & $43(1)$ & $17(1)$ & $-1(1)$ & $-1(1)$ & $3(1)$ \\
\hline$C(11)$ & $50(2)$ & $41(1)$ & $26(1)$ & $6(1)$ & $-2(1)$ & $8(1)$ \\
\hline$C(12)$ & $54(2)$ & $36(1)$ & $28(1)$ & $7(1)$ & $7(1)$ & $-1(1)$ \\
\hline C (13) & $54(2)$ & $37(1)$ & $33(1)$ & $-9(1)$ & $-12(1)$ & $13(1)$ \\
\hline C (14) & $55(1)$ & $28(1)$ & $26(1)$ & $-4(1)$ & $1(1)$ & $7(1)$ \\
\hline$C(15)$ & $43(1)$ & $35(1)$ & $26(1)$ & $-5(1)$ & $-2(1)$ & $-1(1)$ \\
\hline$C(16)$ & $63(2)$ & $49(2)$ & $29(1)$ & $-11(1)$ & $-10(1)$ & $4(1)$ \\
\hline$C(17)$ & $46(1)$ & $54(2)$ & $24(1)$ & $-5(1)$ & $-7(1)$ & $5(1)$ \\
\hline C (18) & $53(2)$ & $47(1)$ & $22(1)$ & $-4(1)$ & $2(1)$ & $-9(1)$ \\
\hline C (19) & $78(2)$ & $39(1)$ & $23(1)$ & $-4(1)$ & $7(1)$ & $-1(1)$ \\
\hline$C(20)$ & $107(3)$ & $56(2)$ & $28(1)$ & $3(1)$ & $10(2)$ & $-21(2)$ \\
\hline$C(21)$ & $35(1)$ & $62(2)$ & $43(2)$ & $0(1)$ & $4(1)$ & $1(1)$ \\
\hline$C(22)$ & $60(2)$ & $34(1)$ & $28(1)$ & $-3(1)$ & $0(1)$ & $7(1)$ \\
\hline C (23) & $87(2)$ & $53(2)$ & $50(2)$ & $-26(2)$ & $-2(2)$ & $15(2)$ \\
\hline
\end{tabular}


Table 5. Hydrogen coordinates ( $\left.\mathrm{x} 10^{\wedge} 4\right)$ and isotropic

displacement parameters $\left(A^{\wedge} 2 \times 10^{\wedge} 3\right)$ for cd25123.

\begin{tabular}{|c|c|c|c|c|}
\hline & $\mathrm{x}$ & $\mathrm{y}$ & z & $\mathrm{U}(\mathrm{eq})$ \\
\hline $\mathrm{H}(1)$ & $2340(30)$ & $4341(19)$ & $7239(8)$ & $23(5)$ \\
\hline $\mathrm{H}(2)$ & $-220(30)$ & $2840(20)$ & $6982(10)$ & $38(6)$ \\
\hline $\mathrm{H}(3)$ & $-920(30)$ & $3560(20)$ & $7520(10)$ & $53(7)$ \\
\hline $\mathrm{H}(4)$ & $-1250(20)$ & $1424(13)$ & $7612(6)$ & $32(4)$ \\
\hline $\mathrm{H}(5)$ & $400(30)$ & $290(20)$ & $8325(9)$ & $32(6)$ \\
\hline $\mathrm{H}(6)$ & $3660(30)$ & $-110(20)$ & $7897(10)$ & $45(6)$ \\
\hline $\mathrm{H}(7)$ & $4310(30)$ & $1200(20)$ & $8027(8)$ & $28(5)$ \\
\hline $\mathrm{H}(8)$ & $3880(30)$ & $790(20)$ & $9610(11)$ & $52(7)$ \\
\hline $\mathrm{H}(9)$ & $5110(30)$ & $890(20)$ & $9075(10)$ & $45(7)$ \\
\hline H (10) & $930(30)$ & $600(20)$ & $9203(10)$ & $37(6)$ \\
\hline $\mathrm{H}(11)$ & $2360(30)$ & $-380(20)$ & $8988(9)$ & $41(6)$ \\
\hline $\mathrm{H}(12)$ & $140(40)$ & $4680(20)$ & $8133(11)$ & $56(7)$ \\
\hline H (13) & $-510(40)$ & $4170(20)$ & $8710(12)$ & $53(8)$ \\
\hline $\mathrm{H}(14)$ & $2530(30)$ & $5850(20)$ & $8575(10)$ & $48(7)$ \\
\hline $\mathrm{H}(15)$ & $4780(30)$ & $4700(20)$ & $8891(9)$ & $36(6)$ \\
\hline $\mathrm{H}(16)$ & $4810(40)$ & $4950(20)$ & $9786(11)$ & $52(8)$ \\
\hline $\mathrm{H}(17)$ & $2700(30)$ & $4290(20)$ & $9861(11)$ & $50(7)$ \\
\hline H (18) & $6240(30)$ & 3133 (18) & $9656(8)$ & $25(5)$ \\
\hline H(19) & $4580(30)$ & $2750(20)$ & $10050(12)$ & $52(7)$ \\
\hline H (20) & $4930(30)$ & $2782(18)$ & $7058(8)$ & $27(5)$ \\
\hline $\mathrm{H}(21)$ & $2070(40)$ & $1320(20)$ & $6720(12)$ & $57(8)$ \\
\hline H (22) & $4180(40)$ & $920(30)$ & $6961(12)$ & $68(9)$ \\
\hline H (23) & $1990(40)$ & $3230(30)$ & $6279(12)$ & $65(9)$ \\
\hline H (24) & $4280(50)$ & $2650(40)$ & $6149(18)$ & $121(15)$ \\
\hline H (25) & $4060(40)$ & $3960(30)$ & $6366(12)$ & $76(9)$ \\
\hline H (26) & $-2340(30)$ & $2730(20)$ & $8434(9)$ & $38(6)$ \\
\hline $\mathrm{H}(27)$ & $-1200(30)$ & $2260(20)$ & $8960(11)$ & $46(6)$ \\
\hline $\mathrm{H}(28)$ & $-2270(40)$ & $1300(30)$ & $8525(10)$ & $58(8)$ \\
\hline H (29) & $-230(40)$ & $7980(30)$ & $9663(11)$ & $58(8)$ \\
\hline $\mathrm{H}(30)$ & $1990(40)$ & $8250(30)$ & $9863(11)$ & $68(9)$ \\
\hline H (31) & $780(40)$ & $7170(30)$ & $10075(15)$ & $78(11)$ \\
\hline
\end{tabular}




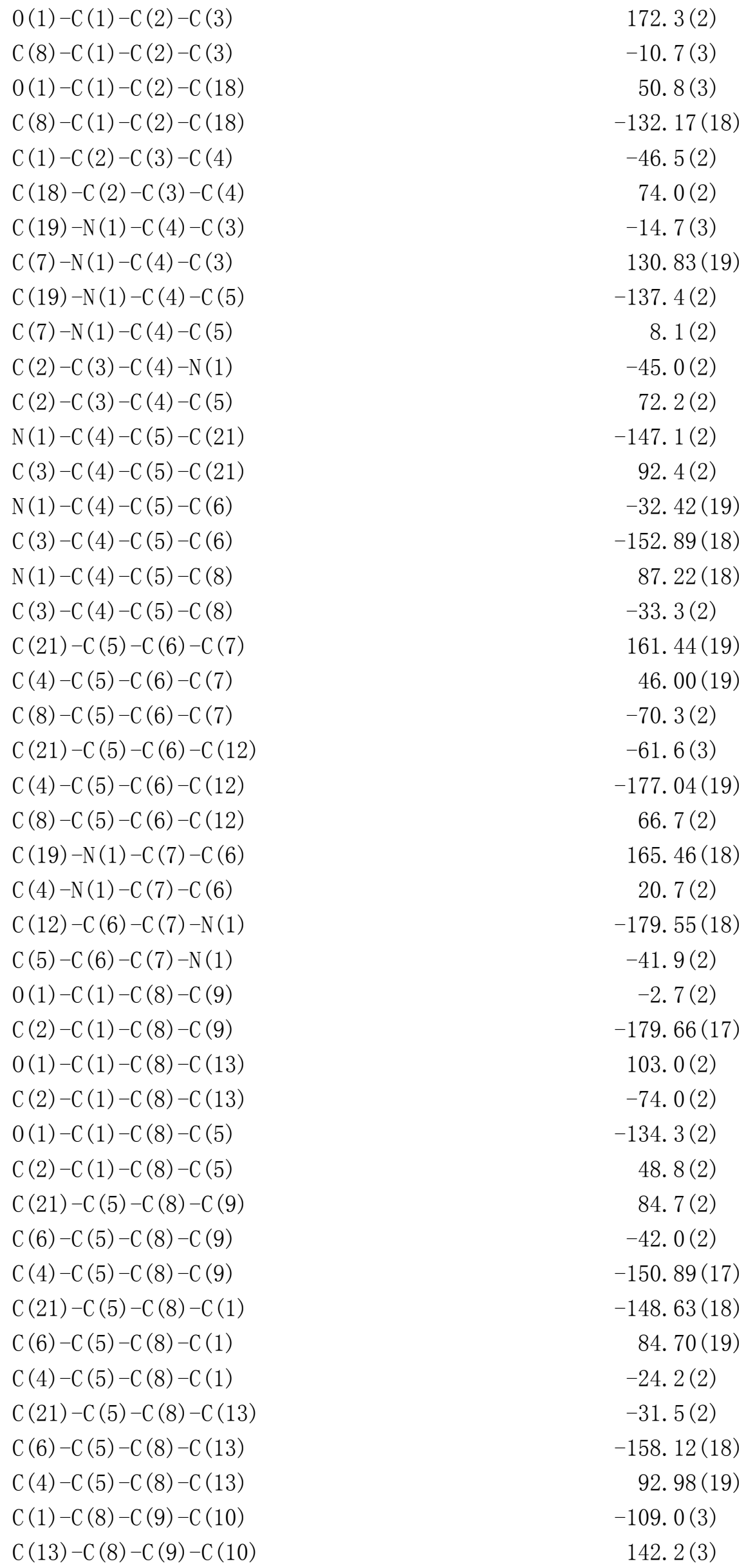




\begin{tabular}{|c|c|}
\hline$C(5)-C(8)-C(9)-C(10)$ & $18.9(3)$ \\
\hline$C(1)-C(8)-C(9)-C(15)$ & 74. $36(19)$ \\
\hline$C(13)-C(8)-C(9)-C(15)$ & $-34.4(2)$ \\
\hline$C(5)-C(8)-C(9)-C(15)$ & $-157.74(17)$ \\
\hline$C(8)-C(9)-C(10)-C(11)$ & $10.0(4)$ \\
\hline$C(15)-C(9)-C(10)-C(11)$ & $-173.4(2)$ \\
\hline$C(8)-C(9)-C(10)-C(17)$ & $-174.9(2)$ \\
\hline$C(15)-C(9)-C(10)-C(17)$ & 1. $7(2)$ \\
\hline$C(9)-C(10)-C(11)-C(12)$ & $-34.3(4)$ \\
\hline$C(17)-C(10)-C(11)-C(12)$ & $151.1(2)$ \\
\hline$C(10)-C(11)-C(12)-C(6)$ & $55.0(3)$ \\
\hline$C(7)-C(6)-C(12)-C(11)$ & $55.5(3)$ \\
\hline$C(5)-C(6)-C(12)-C(11)$ & $-74.9(3)$ \\
\hline$C(9)-C(8)-C(13)-C(14)$ & $31.9(2)$ \\
\hline$C(1)-C(8)-C(13)-C(14)$ & $-80.4(2)$ \\
\hline$C(5)-C(8)-C(13)-C(14)$ & 158. $32(18)$ \\
\hline$C(8)-C(13)-C(14)-C(22)$ & $-140.1(2)$ \\
\hline$C(8)-C(13)-C(14)-C(15)$ & $-18.7(2)$ \\
\hline$C(10)-C(9)-C(15)-C(16)$ & $-22.8(2)$ \\
\hline$C(8)-C(9)-C(15)-C(16)$ & 154. $80(18)$ \\
\hline$C(10)-C(9)-C(15)-C(14)$ & $-153.45(18)$ \\
\hline$C(8)-C(9)-C(15)-C(14)$ & $24.1(2)$ \\
\hline$C(22)-C(14)-C(15)-C(9)$ & $119.6(2)$ \\
\hline$C(13)-C(14)-C(15)-C(9)$ & $-2.4(2)$ \\
\hline$C(22)-C(14)-C(15)-C(16)$ & $2.5(3)$ \\
\hline$C(13)-C(14)-C(15)-C(16)$ & $-119.5(3)$ \\
\hline$C(9)-C(15)-C(16)-C(17)$ & 33. 4(3) \\
\hline$C(14)-C(15)-C(16)-C(17)$ & $151.9(2)$ \\
\hline$C(15)-C(16)-C(17)-C(10)$ & $-32.7(3)$ \\
\hline$C(9)-C(10)-C(17)-C(16)$ & 20. $3(3)$ \\
\hline$C(11)-C(10)-C(17)-C(16)$ & $-164.1(2)$ \\
\hline$C(3)-C(2)-C(18)-C(20)$ & $84.7(3)$ \\
\hline$C(1)-C(2)-C(18)-C(20)$ & $-153.1(2)$ \\
\hline$C(3)-C(2)-C(18)-C(19)$ & $-37.6(3)$ \\
\hline$C(1)-C(2)-C(18)-C(19)$ & 84. 6(2) \\
\hline $\mathrm{C}(4)-\mathrm{N}(1)-\mathrm{C}(19)-\mathrm{C}(18)$ & $52.1(3)$ \\
\hline $\mathrm{C}(7)-\mathrm{N}(1)-\mathrm{C}(19)-\mathrm{C}(18)$ & $-90.2(3)$ \\
\hline $\mathrm{C}(20)-\mathrm{C}(18)-\mathrm{C}(19)-\mathrm{N}(1)$ & $-146.4(3)$ \\
\hline $\mathrm{C}(2)-\mathrm{C}(18)-\mathrm{C}(19)-\mathrm{N}(1)$ & $-22.2(3)$ \\
\hline$C(23)-0(3)-C(22)-0(2)$ & $0.6(4)$ \\
\hline$C(23)-0(3)-C(22)-C(14)$ & $-177.5(2)$ \\
\hline$C(13)-C(14)-C(22)-0(2)$ & 22. 8(3) \\
\hline$C(15)-C(14)-C(22)-0(2)$ & $-94.8(3)$ \\
\hline$C(13)-C(14)-C(22)-0(3)$ & $-159.11(19)$ \\
\hline$C(15)-C(14)-C(22)-0(3)$ & 83. 3(2) \\
\hline
\end{tabular}

Symmetry transformations used to generate equivalent atoms: 
Table 7. Hydrogen bonds for cd25123 [A and deg. ].

D-H. . . A

$\mathrm{d}(\mathrm{D}-\mathrm{H})$

d (H. . . A)

d (D. . . A )

$<(\mathrm{DHA})$ 